WSRC-STI-2007-00697, REV. 0

\title{
CHARACTERIZATION AND ALUMINUM DISSOLUTION DEMONSTRATION WITH A 3 LITER TANK 51H SAMPLE
}

February 2008

Waste Processing Technology Section Savannah River National Laboratory Aiken, SC 29808

Prepared for the U.S. Department of Energy Under Contract Number DEAC09-96SR18500

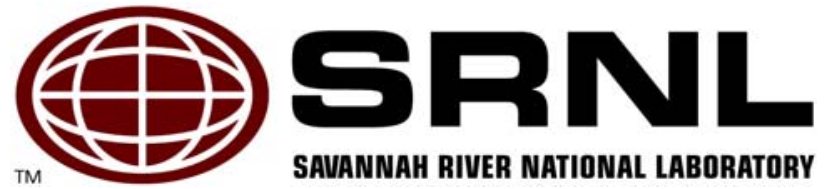




\section{DISCLAIMER}

This report was prepared for the United States Department of Energy under Contract No. DE-AC09-96SR18500 and is an account of work performed under that contract. Neither the United States Department of Energy, nor WSRC, nor any of their employees makes any warranty, expressed or implied, or assumes any legal liability or responsibility for the accuracy, completeness, or usefulness, of any information, apparatus, or product or process disclosed herein or represents that its use will not infringe privately owned rights. Reference herein to any specific commercial product, process, or service by trade name, trademark, name, manufacturer or otherwise does not necessarily constitute or imply endorsement, recommendation, or favoring of same by Westinghouse Savannah River Company or by the United States Government or any agency thereof. The views and opinions of the authors expressed herein do not necessarily state or reflect those of the United States Government or any agency thereof.

\section{Printed in the United States of America}

Prepared For

U.S. Department of Energy 
Key Words:

Sludge Analysis

Supernate Analysis

\title{
Retention: Permanent
}

Key References:

Technical Task Plan: WSRC-RP-2007-00512, Rev. 0

Analytical Study Plan: WSRC-RP-2007-00392, Rev. 2

\section{CHARACTERIZATION AND ALUMINUM DISSOLUTION DEMONSTRATION WITH A 3 LITER TANK 51H SAMPLE}

\author{
M. S. Hay, SRNL/A\&CPT \\ J. M. Pareizs, SRNL/PS\&E \\ C. J. Bannochie, SRNL/PS\&E \\ M. E. Stone, SRNL/PS\&E \\ D. R. Click, SRNL/AD \\ D. J. McCabe, SRNL/A\&CPT
}

Issue Date: February 2008

Waste Processing Technology Section Savannah River National Laboratory Aiken, SC 29808

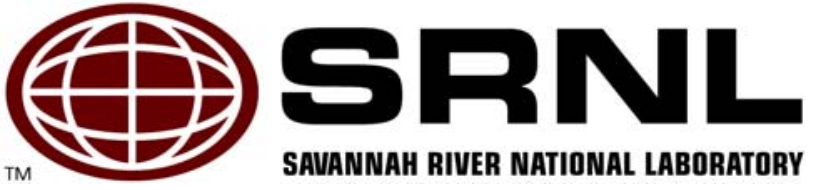




\section{REVIEWS AND APPROVALS}

M. S. Hay, Author, SRNL/A\&CPT

Date

J. M. Pareizs, Author, SRNL/PS\&E

Date

C. J. Bannochie, Author, SRNL/PS\&E

Date

M. E. Stone, Author, SRNL/PS\&E

Date

D. R. Click, Author, SRNL/AD

Date

D. J. McCabe, Author, SRNL/ A\&CPT

Date

C. J. Martino, Technical Reviewer, SRNL/A\&CPT

Date

C. C. Herman, SRNL/ PET, Manager

Date 


\section{TABLE OF CONTENTS}

List of Tables $\quad$ iv

$\begin{array}{ll}\text { List of Figures } & \text { v }\end{array}$

List of Acronyms r vi

SUMMARY vii

$\begin{array}{ll}1.0 \text { INTRODUCTION } & 1\end{array}$

2.0 SAMPLE CHARACTERIZATION AND TEST CONDITIONS 2

2.1 SAMPLE DESCRIPTION

2.2 SUPERNATE SAMPLE PREPARATION FOR ANALYSIS 2

2.3 SLUDGE SAMPLE PREPARATION FOR ANALYSIS 2

2.4 WeIGHT PERCENT SOLIDS AND DENSITY MEASUREMENTS 2

2.5 Aluminum DisSOlution Demonstration TeSt Details 3

\subsection{RESULTS OF THE SAMPLE CHARACTERIZATION 8}

3.1 GENERAL INFORMATION

3.2 Weight PERCENT SOlids AND DENSITY RESUlts Of THE As-RECEIVED SLUDGE SLURRY SAMPLE 9

3.3 ANALYTICAL RESULTS FOR THE AS-RECEIVED FILTERED SUPERNATE 9

3.4 ANALYTICAL RESULTS For THE As-RECEIVED TANK 51H SLUDGE SOLIDS 9

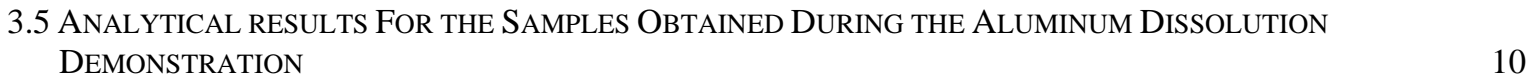

3.6 Weight Percent Solids and Density Results of the Post Aluminum DisSOlution SLudge SluRry

3.7 ANAlyticAl RESUlts For the Post Aluminum DisSOlution Filtered SUPERNATE 10

3.8 ANALYTICAl RESUlts For THE POST Aluminum DisSOLUTION SLUDGE SOLIDS 11

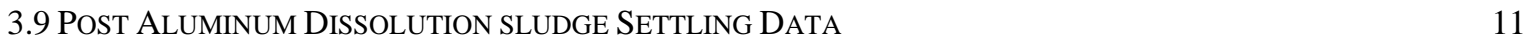

\subsection{DISCUSSION OF RESULTS 35}

4.1 AS-RECEIVED TANK 5IH SLUDGE SAMPLE CHARACTERIZATION 35

4.2 ALUMINUM DISOLUTION DEMONSTRATION RESULTS 36

4.3 SLUDGE SETTLing AFTER Aluminum DisSOLUTION

4.4 Post Aluminum DisSOlution SLUDGE AND SUPERNATE CHARACTERIZATION 39

4.5 CONCLUSIONS $\quad 41$

$\begin{array}{ll}5.0 \text { REFERENCES } & 43\end{array}$ 


\section{List of Tables}

TABLE 2-1. COMPOSITION OF THE ANALYTICAL REFERENCE GLASS-1 STANDARD. ......................................5

TABLE 2-2. COMPOSITION OF THE SIMULATED TANK 11H SUPERNATE.........................................................5

TABLE 3-1. ABBREVIATIONS FOR ANALYTICAL METHODS USED IN DATA TABLES .........................................8

TABle 3-2. WeIGHT PERCENT Solids AND DENSITY OF THE As-RECEIVED TANK 51H SLUDGE SAMPLE .....12

TABLE 3-3. COMPOSITION OF THE As-RECEIVED TANK 51H FILTERED SuPERNATE ...................................13

TABle 3-4. COMposition of the Aqua Regia Dissolution of Total Dried Solids From As-RecEIVED TANK 51H SLUDGE ....................................................................................................16

TABle 3-5. COMPOSITION OF THE PEROXIDE Fusion DisSOLUTION OF TOTAL DRIED SOLIDS FROM AsRECEIVED TANK 51H SLUDGE .................................................................................... 18

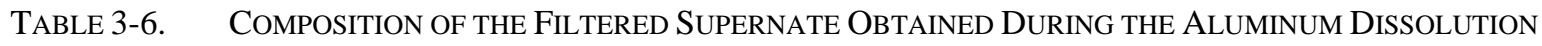

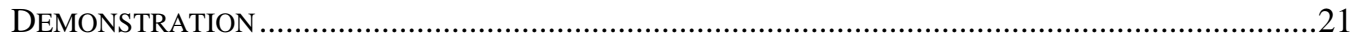

TABle 3-7. Weight Percent Solids And Density of The Post Aluminum Dissolution TANK 51H

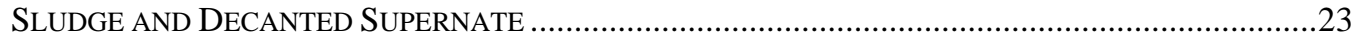

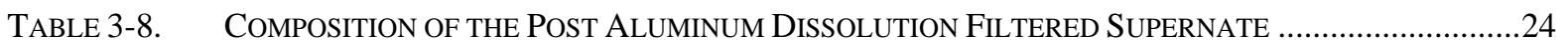

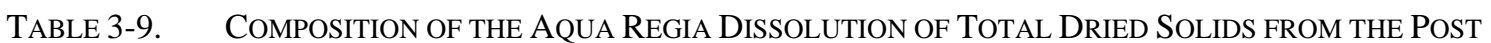
ALUMINUM DISSOLUTION SLUDGE ..................................................................................28

Table 3-10. Composition of the Peroxide Fusion Dissolution of Total Dried Solids From the Post

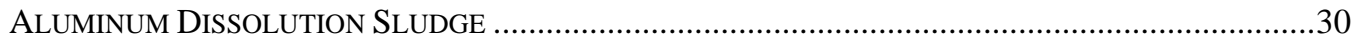

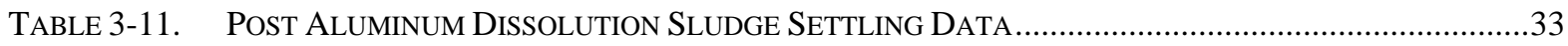

TABLE 4-1. SUMMARY OF THE AS-RECEIVED TANK 51H SLUDGE COMPOSITION.........................................35

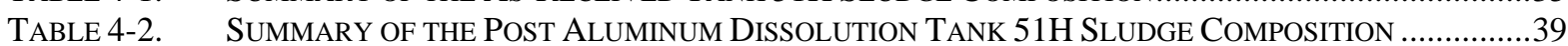

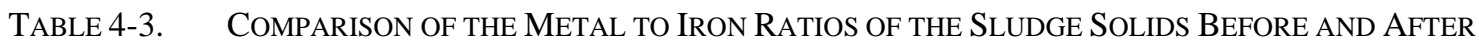

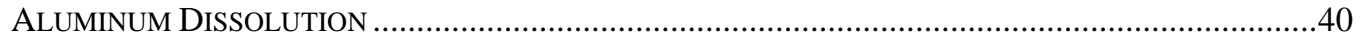

TABle 4-4. COMPARISON OF RADIONUClide to IRON RATIOS OF THE SLUDGE SOlIDS BEFORE AND AFTER

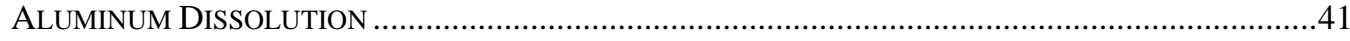




\section{List of Figures}

FIGURE 2.1 ALUMINUM DisSOLUTION EQUIPMENT IN THE SHIELDED CELLS...............................................6

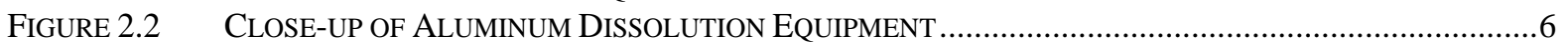

FIGURE 2.3 SCHEMATIC OF ALUMINUM DiSSOLUTION EQUIPMENT (NOT TO SCALE) .....................................

FIgURE 3.1 X-RAY DifFRACTION FOR THE AS-RECEIVED TANK 51H SLUDGE SOLIDS ................................34

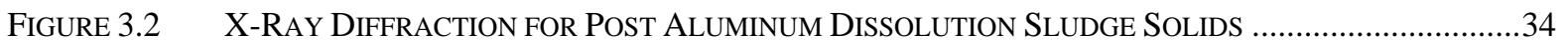

Figure 4.1 ALUMinUm CONCENTRATION IN SOLUTION DURING THE ALUMinUm DisSOLUTION

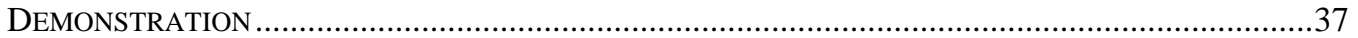

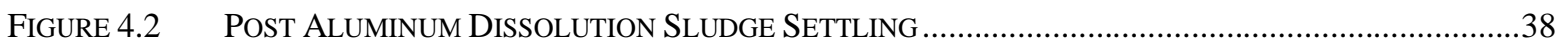

FIGURE 4.3 COMPARISON OF SLUDGE SETTLING FOR HIGH ALUMINUM SLUDGE ........................................38 


\section{List of Acronyms}

$\begin{array}{ll}\text { AD } & \text { Analytical Development } \\ \text { ARG1 } & \text { Analytical Reference Glass 1 } \\ \text { CPC } & \text { Chemical Process Cell } \\ \text { CSEM } & \text { Contained Scanning Electron Microscopy } \\ \text { DWPF } & \text { Defense Waste Processing Facility } \\ \text { EDX } & \text { Energy Dispersive X-ray } \\ \text { HM } & \text { H-Area Modified PUREX } \\ \text { IC } & \text { Ion Chromatography } \\ \text { ICP-ES } & \text { Inductively Couple Plasma-Emission Spectroscopy } \\ \text { ICP-MS } & \text { Inductively Couple Plasma-Mass Spectrometry } \\ \text { LWO } & \text { Liquid Waste Organization } \\ \text { PNNL } & \text { Pacific Northwest National Laboratory } \\ \text { \%RSD } & \text { Percent Relative Standard Deviation } \\ \text { PUREX } & \text { Plutonium Uranium Reduction Extraction } \\ \text { SB5 } & \text { Sludge Batch 5 } \\ \text { SRNL } & \text { Savannah River National Laboratory } \\ \text { SRS } & \text { Savannah River Site } \\ \text { TIC } & \text { Total Inorganic Carbon } \\ \text { TTQAP } & \text { Task Technical and Quality Assurance Plan } \\ \text { TTR } & \text { Technical Task Request } \\ \text { XRD } & \text { X-ray Diffraction }\end{array}$




\section{SUMMARY}

A 3-liter sludge slurry sample was sent to SRNL for demonstration of a low temperature aluminum dissolution process. The sludge was characterized before and after the aluminum dissolution. Post aluminum dissolution sludge settling and the stability of the decanted supernate were also observed.

The characterization of the as-received 3-liter sample of Tank $51 \mathrm{H}$ sludge slurry shows a typical high aluminum HM sludge. The XRD analysis of the dried solids indicates Boehmite is the predominant crystalline form of aluminum in the sludge solids. However, amorphous phases of aluminum present in the sludge would not be identified using this analytical technique.

The low temperature $\left(55^{\circ} \mathrm{C}\right)$ aluminum dissolution process was effective at dissolving aluminum from the sludge. Over the three week test, $\sim 42 \%$ of the aluminum was dissolved out of the sludge solids. The process appears to be selective for aluminum with no other metals dissolving to any appreciable extent. At the termination of the three week test, the aluminum concentration in the supernate had not leveled off indicating more aluminum could be dissolved from the sludge with longer contact times or higher temperatures. The slow aluminum dissolution rate in the test may indicate the dissolution of the Boehmite form of aluminum however; insufficient kinetic data exists to confirm this hypothesis.

The aluminum dissolution process appears to have minimal impact on the settling rate of the post aluminum dissolution sludge. However, limited settling data were generated during the test to quantify the effects. The sludge settling was complete after approximately twelve days.

The supernate decanted from the settled sludge after aluminum dissolution appears stable and did not precipitate aluminum over the course of several months. A mixture of the decanted supernate with Tank 11 simulated supernate was also stable with respect to precipitation. 


\subsection{INTRODUCTION}

Current plans call for the contents of Tank 51H to be blended with Plutonium Uranium Reduction Extraction (PUREX) sludge from Tank 7F and the sludge heel in Tank 40H (SB4) to constitute Sludge Batch 5 (SB5) feed to the Defense Waste Processing Facility (DWPF). The Savannah River Site (SRS) Liquid Waste Organization (LWO) intends to add caustic to the HM sludge in Tank $51 \mathrm{H}$ to dissolve aluminum, thereby reducing the total mass of sludge solids being fed to the DWPF. A small-scale demonstration was completed with a $\sim 30 \mathrm{~mL}$ sample to ascertain the viability of the low temperature $\left(55^{\circ} \mathrm{C}\right)$ aluminum dissolution process. However, before performing low temperature aluminum dissolution in the Tank Farm, a larger-scale radioactive demonstration using a 3-liter Tank 51H sludge sample was necessary to determine potential downstream impacts so technical issues could be identified before the start of SB5 processing. Downstream impacts include sludge washing and settling, DWPF Chemical Process Cell (CPC) and melter processing envelopes. A Task Technical and Quality Assurance Plan (TTQAP) describes the requirements for the demonstration. ${ }^{1}$ The test conditions for the demonstration attempted to match those documented in the flowsheet as closely as possible. ${ }^{2}$ An Analytical Study Plan was developed to define the sampling and analytical needs during the demonstration. ${ }^{3}$

This work is Technical Baseline Research and Development for the Department of Energy Office of Cleanup Technologies (EM-21). The Savannah River National Laboratory (SRNL) received funding to perform a radioactive demonstration using Tank 51H sludge in the SRNL Shielded Cells Facility. This report documents the aluminum dissolution demonstration and the characterization of the sludge before and after aluminum dissolution. The sludge washing

and evaluation of DWPF impacts, along with some additional characterization data, will be documented in a separate report. 
WSRC-STI-2007-00697, REV. 0

\subsection{SAMPLE CHARACTERIZATION AND TEST CONDITIONS}

\subsection{SAMPLE DESCRIPTION}

A 3-liter sludge slurry sample (ID No. = HTF-51-07-77) from Tank 51H was sent to SRNL for characterization and demonstration of a low temperature aluminum dissolution process. The sample was received at SRNL on May 31, 2007. The sludge slurry sample was mixed and pumped from the sample container. The sludge slurry sample was allowed to settle overnight after which clear supernate was pumped back into the sample container to rinse out any remaining sludge solids. Approximately $3280 \mathrm{~g}(\sim 3.04 \mathrm{~L})$ of a sludge solids/supernate mixture was recovered from the sample container.

\subsection{SUPERNATE SAMPLE PREPARATION FOR ANALYSIS}

A portion of the well-mixed sludge slurry was filtered through a $0.45 \mu$ porosity filter. Portions of the filtered supernate were diluted with de-ionized distilled water or nitric acid to reduce the sample activity and allow removal from the Shielded Cells for chemical analysis. All sample preparations of the filtered supernate samples were conducted in quadruplicate. A blank and applicable standard were prepared along with the sample dilutions.

\subsection{SLUDGE SAMPLE PREPARATION FOR ANALYSIS}

Sludge solids for X-Ray Diffraction (XRD) analysis were obtained by filtration of the sludge slurry. After filtration a small amount of de-ionized water was used to rinse any residual supernate out of the sludge solids. The washed solids collected on the filter were allowed to air-dry. The air-dried solids were weighed into a shielded bottle and sent for analysis.

Portions of the sludge slurry were dried in an oven at $100{ }^{\circ} \mathrm{C}$. The dried solids were prepared for analysis using two methods; dissolution in aqua regia, and fusion with sodium peroxide followed by uptake in nitric acid. The digested solids were diluted to reduce activity and allow removal from the Shielded Cells. All sample preparations were conducted in quadruplicate. Triplicate digestions of a glass standard containing many of the elements found in tank waste sludge samples were prepared concurrently with the sample digestions for check for the completeness of preparation. Table 2-1 lists the composition of the Analytical Reference Glass-1 (ARG-1) glass standard. ${ }^{4}$ A blank and applicable standards were prepared along with the sample digestions.

\subsection{WEIGHT PERCENT SOLIDS AND DENSITY MEASUREMENTS}

The densities of the filtered supernate and the well-mixed slurry sample were measured in the Shielded Cells using calibrated plastic tubes with a nominal volume of $\sim 8.25 \mathrm{~mL}$. The density measurements were conducted in quadruplicate on each phase of the sample.

The weight percent total solids in the slurry sample were measured in the Shielded Cells using a conventional drying oven at $110^{\circ} \mathrm{C}$. The sample was dried until repeated weights 
indicated no further loss of water. The weight percent dissolved solids in a sample of the filtered supernate were measured in the same manner. All weight percent solids measurements were made in quadruplicate. The weight percent insoluble solids and weight percent soluble solids in the slurry sample were calculated using the equations shown below.

Wis $=($ Wts - Wds $) /(1-W d s) \quad$ and $\quad$ Wss $=$ Wts - Wis

where:

Wis $=$ weight fraction of insoluble solids in the slurry

Wss = weight fraction of soluble solids in the slurry

Wts = weight fraction of total solids in the slurry

Wds = weight fraction of dissolved solids in the filtered supernate

Thus:

$\mathrm{Wt} \%$ dissolved solids $=\quad$ (wt dissolved solids/wt of supernate $) \times 100$

$\mathrm{Wt} \%$ total solids $=\quad$ (wt total solids/wt of total slurry) $\mathrm{x} 100$

$\mathrm{Wt} \%$ insoluble solids $=\quad$ (wt insoluble solids/wt of total slurry) $\mathrm{x} 100$

$\mathrm{Wt} \%$ soluble solids $=\quad(\mathrm{wt}$ of dissolved solids/wt of total slurry) $\times 100$

\subsection{ALUMINUM DISSOLUTION DEMONSTRATION TEST DETAILS}

A total of $2678 \mathrm{~g}$ ( $2400 \mathrm{~mL})$ of Tank $51 \mathrm{H}$ sludge slurry was pumped into a specially designed reaction vessel. Figures 2.1 and 2.2 show photographs of the reaction vessel. Figure 2.3 contains a labeled schematic of the aluminum dissolution equipment. After initiating mixing, $1013 \mathrm{~g}$ ( $\sim 660 \mathrm{~mL})$ of $50 \mathrm{wt} \% \mathrm{NaOH}$ was added to the vessel over a period of approximately one hour. The temperature of the mixture in the reaction vessel increased from $\sim 25^{\circ} \mathrm{C}$ to $\sim 40{ }^{\circ} \mathrm{C}$ over the course of the $\mathrm{NaOH}$ addition. After addition of the $\mathrm{NaOH}$, the reaction vessel was heated to $55^{\circ} \mathrm{C}$ using a water bath. The reaction vessel was maintained at $55^{\circ} \mathrm{C}$ for three weeks. During this time, small aliquots $(\sim 5 \mathrm{~mL})$ were removed from the reaction vessel approximately every other day. The aliquots were immediately filtered through a $0.45 \mu$ filter. The eleven filtrates obtained were diluted in nitric acid and sent for analysis.

The temperature for the test was constrained to $55^{\circ} \mathrm{C}$ because the actual aluminum dissolution in Tank $51 \mathrm{H}$ will be limited to a maximum temperature of $65^{\circ} \mathrm{C}$. The tank temperature limit results primarily from the temperature limit of the slurry pump seals.

After the three week reaction time, mixing in the reaction vessel was stopped and the temperature of the water bath was lowered to maintain a temperature inside the reaction vessel of $35^{\circ} \mathrm{C}$. The sludge was allowed to settle undisturbed for three weeks at $35^{\circ} \mathrm{C}$. The level of the sludge/supernate interface inside the reaction vessel was recorded periodically. At the completion of the settling phase, the supernate was decanted from the vessel to as close to the settled sludge interface as possible without disturbing the sludge surface. Approximately $1454 \mathrm{~g}$ ( $1240 \mathrm{~mL})$ of supernate were decanted from the sludge. Samples of 
the decanted supernate and remaining sludge were prepared for analysis in the same manner described above.

A small portion of the decanted supernate $(\sim 200 \mathrm{~mL})$ was poured into a bottle containing 13 $\mathrm{mL}$ of a simulated Tank $11 \mathrm{H}$ supernate to simulant the transfer of the decant from Tank $51 \mathrm{H}$ to Tank $11 \mathrm{H}$ as described in the flowsheet. The decanted supernate from the aluminum dissolution and the mixture of the decanted supernate with the simulated Tank $11 \mathrm{H}$ supernate were observed for solids formation for several months at ambient temperature in the Shielded Cells $\left(\sim 18-25^{\circ} \mathrm{C}\right)$. No solids formation was observed in either bottle. Table 2.2 shows the composition of the simulated Tank $11 \mathrm{H}$ supernate used in the mixing test. 
Table 2-1. Composition of the Analytical Reference Glass-1 Standard.

\begin{tabular}{|l|c|c|}
\hline Element & wt \% in Glass & $\mathbf{~ m g / k g ~ i n ~ G l a s s ~}$ \\
\hline $\mathrm{Al}$ & 2.50 & $2.50 \mathrm{E}+04$ \\
\hline $\mathrm{B}$ & 2.69 & $2.69 \mathrm{E}+04$ \\
\hline $\mathrm{Ba}$ & 0.079 & $7.90 \mathrm{E}+02$ \\
\hline $\mathrm{Ca}$ & 1.02 & $1.02 \mathrm{E}+04$ \\
\hline $\mathrm{Cr}$ & 0.064 & $6.40 \mathrm{E}+02$ \\
\hline $\mathrm{Cu}$ & 0.003 & $3.0 \mathrm{E}+01$ \\
\hline $\mathrm{Fe}$ & 9.79 & $9.79 \mathrm{E}+04$ \\
\hline $\mathrm{K}$ & 2.26 & $2.26 \mathrm{E}+04$ \\
\hline $\mathrm{Li}$ & 1.49 & $1.49 \mathrm{E}+04$ \\
\hline $\mathrm{Mg}$ & 0.52 & $5.2 \mathrm{E}+03$ \\
\hline $\mathrm{Mn}$ & 1.46 & $1.46 \mathrm{E}+04$ \\
\hline $\mathrm{Na}$ & 8.52 & $8.52 \mathrm{E}+04$ \\
\hline $\mathrm{Ni}$ & 0.83 & $8.3 \mathrm{E}+03$ \\
\hline $\mathrm{P}$ & 0.11 & $1.1 \mathrm{E}+03$ \\
\hline $\mathrm{Si}$ & 22.4 & $2.24 \mathrm{E}+05$ \\
\hline $\mathrm{Sr}$ & 0.003 & $3.0 \mathrm{E}+01$ \\
\hline $\mathrm{Ti}$ & 0.69 & $6.9 \mathrm{E}+03$ \\
\hline $\mathrm{Zn}$ & 0.016 & $1.6 \mathrm{E}+02$ \\
\hline $\mathrm{Zr}$ & 0.096 & $9.6 \mathrm{E}+02$ \\
\hline
\end{tabular}

Table 2-2. Composition of the Simulated Tank $11 \mathrm{H}$ Supernate.

\begin{tabular}{|l|c|}
\hline Analyte & M \\
\hline $\mathrm{NO}_{3}{ }^{-}$ & 0.245 \\
\hline $\mathrm{NO}_{2}{ }^{-}$ & 0.13 \\
\hline $\mathrm{OH}^{-}$ & 0.005 \\
\hline $\mathrm{Na}^{+}$ & 0.38 \\
\hline
\end{tabular}




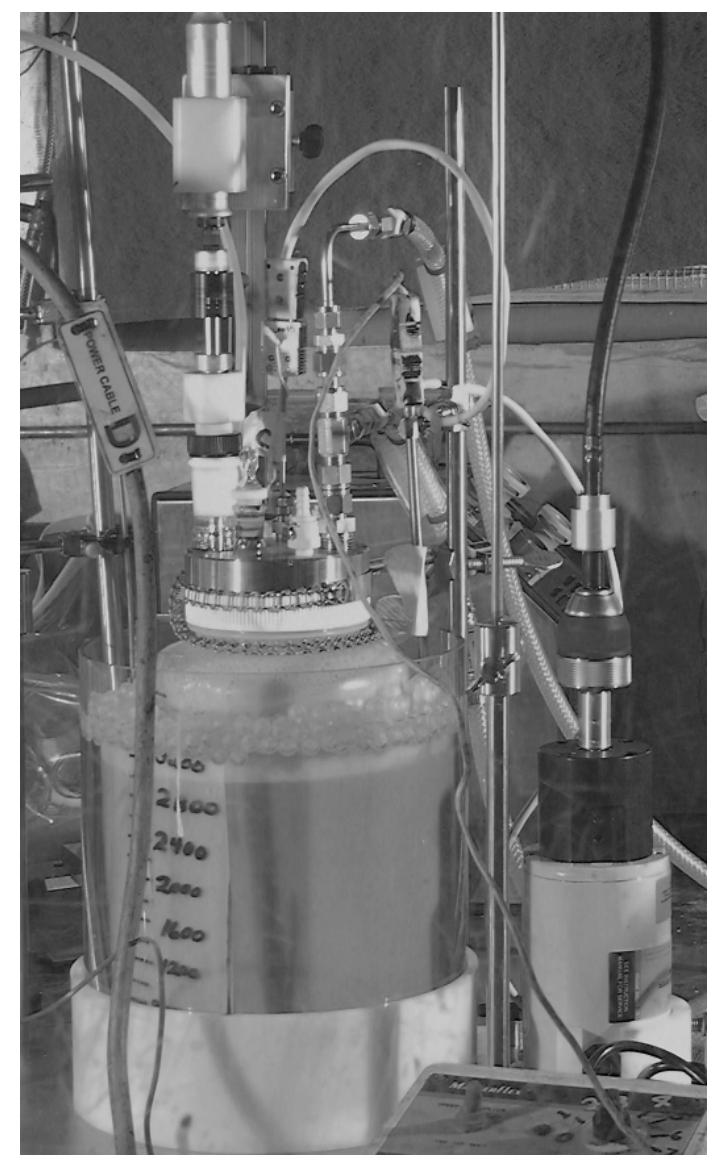

Figure 2.1 Aluminum Dissolution Equipment in the Shielded Cells

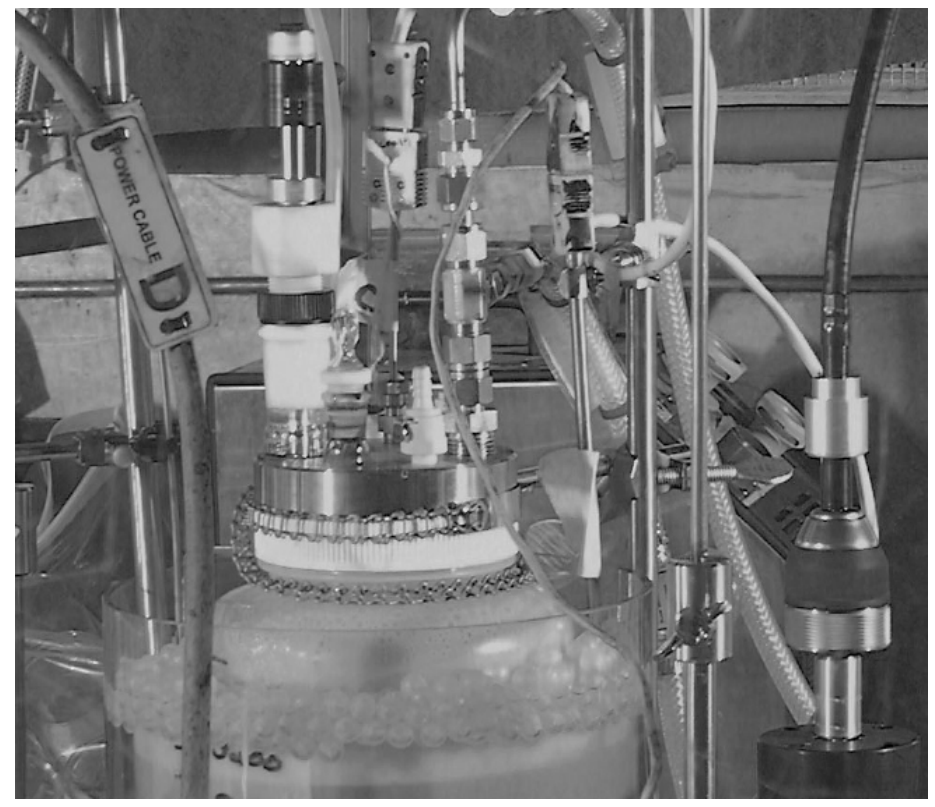

Figure 2.2 Close-up of Aluminum Dissolution Equipment 


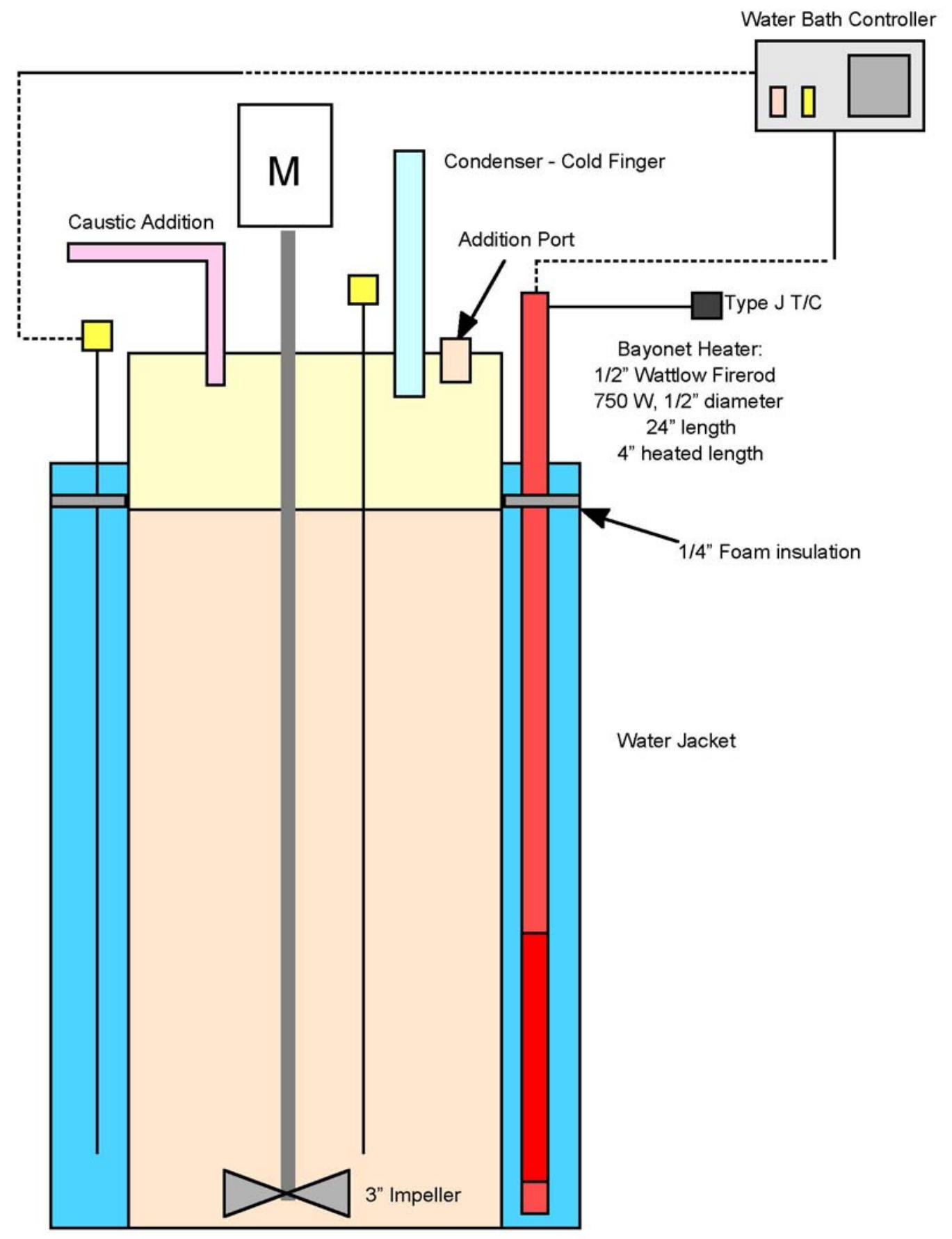

Figure 2.3 Schematic of Aluminum Dissolution Equipment (Not to Scale) 


\subsection{RESULTS OF THE SAMPLE CHARACTERIZATION}

\subsection{GENERAL INFORMATION}

Tables 3-2 through 3-10 provide the composition of the Tank 51H sample received at SRNL and samples generated from the Aluminum Dissolution Demonstration. Table 3.11 shows the settling data for the post aluminum dissolution sludge. Figures 3.1 and 3.2 provide the results of the XRD analysis of the samples. Portions of these results were reported previously. ${ }^{5,6}$

The tables include the results of all replicates, blanks, and glass standards. In tables containing data for digested solids (or slurry) samples, the blank has been converted to solids basis to allow direct comparison to the sample data as a quality indicator. The data for the blank were converted to a solids basis by dividing the concentrations measured in the blank by the target weight of solids used in the digestion ( $\sim 0.250 \mathrm{~g}$ in most cases). For waste tank sample characterization, an uncertainty of approximately $+/-15 \%$ has been found to be the normal range for the combined sampling and analytical uncertainty. ${ }^{7}$ For the Tank $51 \mathrm{H}$ samples, the percent relative standard deviation (\%RSD) presented in the tables only includes the uncertainty associated with sub-sampling in the Shielded Cells and the uncertainty of the analytical method. It should be noted that the samples represent a small amount of material from a large tank.

The data tables also indicate the analytical method used to measure each analyte. Table 3-1 shows the abbreviations used for each analytical method:

Table 3-1. Abbreviations for Analytical Methods used in Data Tables

\begin{tabular}{|l|c|}
\hline Analytical Method & $\begin{array}{c}\text { Abbreviation } \\
\text { in Tables }\end{array}$ \\
\hline Inductively Coupled Plasma-Emission Spectroscopy & IE \\
\hline Inductively Coupled Plasma-Mass Spectrometry & IM \\
\hline Ion Chromatography & IC \\
\hline Titration & T \\
\hline Total Inorganic Carbon & TIC \\
\hline Atomic Absorption Cold Vapor Method & CV \\
\hline Separation/Gamma Spec. & SG \\
\hline Separation/Alpha Spec. & SA \\
\hline Separation/Liquid Scintillation & SL \\
\hline Alpha Counting & AC \\
\hline
\end{tabular}


WSRC-STI-2007-00697, REV. 0

\subsection{WEIGHT PERCENT SOLIDS AND DENSITY RESULTS OF THE AS- RECEIVED SLUDGE SLURRY SAMPLE}

Table 3-2 shows the results of weight percent solids and density measurements made of the as-received Tank $51 \mathrm{H}$ sludge slurry sample and the filtered supernate obtained from that sample.

\subsection{ANALYTICAL RESULTS FOR THE AS-RECEIVED FILTERED SUPERNATE}

Table 3-3 shows the results of the analysis of the as-received Tank $51 \mathrm{H}$ filtered supernate. The cation ( $\mathrm{Na}, \mathrm{K}$ ) equivalents in the supernate sum to $1.05 \mathrm{M}$ while the anion equivalents $\left(\mathrm{NO}_{3}{ }^{-}, \mathrm{NO}_{2}{ }^{-}, \mathrm{SO}_{4}{ }^{2-}, \mathrm{C}_{2} \mathrm{O}_{4}{ }^{2-}, \mathrm{Cl}^{-}, \mathrm{F}^{-}, \mathrm{OH}^{-}\right.$, and $\mathrm{AlO}_{2}{ }^{-}$) sum to $0.90 \mathrm{M}$ for a difference of $\sim 15 \%$. The $0.15 \mathrm{M}$ difference between the sums can be attributed, at least in part, to carbonate anion, which was not measured. The free hydroxide and ${ }^{241} \mathrm{Pu}$ show high uncertainties probably resulting from the concentrations being close to the detection limits of the method. Only aluminum, sodium, and nickel were detected by Inductively Coupled Plasma-Emission Spectroscopy (ICP-ES). One replicate in the ICP-ES results was dropped from the calculation of the average since the values for that replicate were over $30 \%$ outside the average for the other three replicates. The blank shows low levels of plutonium contamination. Assuming the same magnitude of contamination in the sample replicates, the contamination levels should not impact the plutonium results for the sample.

\subsection{ANALYTICAL RESULTS FOR THE AS-RECEIVED TANK 51H SLUDGE SOLIDS}

Tables 3-4 through 3-5 show the results of the analysis of the aqua regia and sodium peroxide fusion dissolutions of the total dried solids obtained from the as-received Tank $51 \mathrm{H}$ sludge sample (HTF-51-07-77). The aqua regia and sodium peroxide fusion data show good agreement between the two dissolution methods for the major components of the sludge solids with the exception of the results for aluminum. A much lower ( 2X) aluminum concentration was obtained from the aqua regia dissolved solids. Prior experience with high aluminum sludge from Tank $11 \mathrm{H}$ found that the aqua regia dissolution method will not consistently dissolve all of the aluminum. ${ }^{8}$ Therefore, only the aluminum results obtained from the peroxide fusion dissolved solids should be used. The ${ }^{239 / 240} \mathrm{Pu}$ results by Inductively Coupled Plasma-Mass Spectroscopy (ICP-MS) and alpha counting show good agreement with a difference of only $\sim 10 \%$.

Figure 3.1 shows the XRD results for the as-received sludge solids. The results indicate Boehmite as the predominant crystalline form of aluminum present in the solids. The iron compound Hematite and uranium compound Clarkeite appear as the next most abundant materials. The XRD results indicate good agreement with the results of the chemical analysis in Tables 3-4 and 3-5 that showed aluminum as the most abundant metal followed by iron, manganese, and uranium. The Muscovite shown in the XRD spectrum results from an impurity present in the water at SRS and appears in nearly all XRD results. 
WSRC-STI-2007-00697, REV. 0

\subsection{ANALYTICAL RESULTS FOR THE SAMPLES OBTAINED DURING THE ALUMINUM DISSOLUTION DEMONSTRATION}

Table 3-6 shows the result of the analysis of samples obtained during the aluminum dissolution demonstration. Only a single replicate at each time period was analyzed and no blanks or standards were submitted concurrently with the samples. The time-zero values were estimated by adjusting the as-received filtered supernate composition from Table 3-3 for the volume of $\mathrm{NaOH}$ added at the beginning of the aluminum dissolution demonstration. Some measure of the data quality can be deduced from the measured sodium concentration. From the one-hour sample onward the sodium concentration should remain constant throughout the test assuming no precipitation occurs. The sodium data in Table 3-6 show very little variation over the course of the test indicating reasonable data quality. However, the plutonium data by ICP-MS and alpha counting show poor agreement most probably the result of the concentrations being very close to the detection limits in the ICP-MS.

\subsection{WEIGHT PERCENT SOLIDS AND DENSITY RESULTS OF THE POST ALUMINUM DISSOLUTION SLUDGE SLURRY}

Table 3-7 shows the results of weight percent solids and density measurements made on the post aluminum dissolution sludge slurry sample and the filtered supernate. Due to the addition of a large volume of concentrated sodium hydroxide solution for the aluminum dissolution test, the weight percent total solids and weight percent dissolved solids increased significantly.

\subsection{ANALYTICAL RESULTS FOR THE POST ALUMINUM DISSOLUTION FILTERED SUPERNATE}

Table 3-8 shows the analytical results for the filtered supernate obtained from the slurry after completion of the aluminum dissolution demonstration and sludge settling. The results represent the composition of the material decanted from the settled sludge at the completion of aluminum dissolution. The data quality appears good with low \%RSD's for the replicates and no significant radioactive contamination present in the blanks. Several analytes were measured using more than one analytical method providing another indication of data quality. In all cases the agreement between methods was good. Sodium was measured by both ICP-ES and Atomic Absorption (AA) with the results showing a difference of $\sim 8 \%$. The sulfate result, as measured by Ion Chromatography (IC), shows good agreement with the sulfur result from ICP-ES indicating a difference of $\sim 8 \%$ also. The ${ }^{137} \mathrm{Cs}$ as measured by ICP-MS and gamma counting shows a difference of only $~ 5 \%$. The plutonium results from ICP-MS and alpha counting show reasonable agreement ( $16 \%$ difference) considering that the plutonium concentration in the filtered supernate appears to very close to the ICP-MS detection limit. The sum of the cation equivalents versus the anion equivalents in the supernate shows a small difference ( $\sim \%$ ) likely resulting from the undetermined carbonate anion concentration. Nitrate, nitrite, and sulfate concentrations closely match the expected diluted concentration based on the volume of concentrated sodium hydroxide added to the asreceived sludge. 


\subsection{ANALYTICAL RESULTS FOR THE POST ALUMINUM DISSOLUTION SLUDGE SOLIDS}

Tables 3-9 through 3-10 show the results of the analysis of the aqua regia and sodium peroxide fusion dissolutions on a total dried solids basis obtained from the post aluminum dissolution sludge after settling and decanting the supernate. The aqua regia and sodium peroxide fusion data show good agreement between the two dissolution methods for the major components of the sludge solids with the exception of the results for aluminum. Prior experience indicates that the aqua regia dissolution method will not consistently dissolve all of the aluminum. ${ }^{8}$ Therefore; only the aluminum results obtained from the peroxide fusion dissolved solids should be used. The ${ }^{239 / 240} \mathrm{Pu}$ results by ICP-MS and alpha counting show good agreement with a difference of only $\sim 4 \%$.

Figure 3.2 shows the XRD results for the post aluminum dissolution sludge solids. Although a significant amount of aluminum dissolved from the sludge during the aluminum dissolution demonstration, the results again show Boehmite as the predominant crystalline phase present in the solids. The iron compound Hematite appears as the next most abundant material. The XRD results indicate good agreement with the results of the chemical analysis in Tables 3-9 and 3-10 that showed aluminum as the most abundant metal followed by iron, manganese, and uranium.

\subsection{POST ALUMINUM DISSOLUTION SLUDGE SETTLING DATA}

Table 3-11 shows the data collected during the post aluminum dissolution sludge settling. The sludge settled with a clear interface between the settling sludge solids and the clear supernate phase above. The sludge stopped settling after approximately 285 hours ( 12 days) but settling data were collected for 21 days as planned. 
WSRC-STI-2007-00697, REV. 0

Table 3-2. Weight Percent Solids and Density of the As-Received Tank 51H Sludge Sample

\begin{tabular}{|c|c|c|c|c|c|c|c|}
\hline Analyte (Method) & Units & $\begin{array}{c}\text { 1st } \\
\text { Replicate }\end{array}$ & $\begin{array}{c}\text { 2nd } \\
\text { Replicate }\end{array}$ & $\begin{array}{c}\text { 3rd } \\
\text { Replicate }\end{array}$ & $\begin{array}{c}\text { 4th } \\
\text { Replicate }\end{array}$ & Average & \%RSD \\
\hline Weight Percent Total Solids & $\mathrm{Wt} \%$ & 15.5 & 15.5 & 15.5 & 15.5 & 15.5 & $0.2 \%$ \\
\hline Weight Percent Dissolved Solids & $\mathrm{Wt} \%$ & 6.73 & 6.74 & 6.74 & 6.79 & 6.75 & $0.4 \%$ \\
\hline Weight Percent Soluble Solids & $\mathrm{Wt} \%$ & - & - & - & - & $6.12 *$ & - \\
\hline Weight Percent Insoluble Solids & $\mathrm{Wt} \%$ & - & - & - & - & 9.39* & - \\
\hline Density of Slurry & $\mathrm{g} / \mathrm{mL}$ & 1.07 & 1.07 & 1.08 & 1.08 & 1.08 & $0.5 \%$ \\
\hline Density of Supernate & $\mathrm{g} / \mathrm{mL}$ & 1.07 & 1.06 & 1.04 & 1.05 & 1.06 & $1.1 \%$ \\
\hline
\end{tabular}

* Values for the weight percent soluble solids and weight percent insoluble solids were calculated from the measured weight percent total solids and weight percent dissolved solids (see Section 2.4) 
WSRC-STI-2007-00697, REV. 0

Table 3-3. Composition of the As-Received Tank 51H Filtered Supernate

\begin{tabular}{|c|c|c|c|c|c|c|c|c|}
\hline Analyte (Method) & Units & $\begin{array}{c}\text { 1st } \\
\text { Replicate }\end{array}$ & $\begin{array}{c}\text { 2nd } \\
\text { Replicate }\end{array}$ & $\begin{array}{c}\text { 3rd } \\
\text { Replicate } \\
\end{array}$ & $\begin{array}{c}\text { 4th } \\
\text { Replicate }\end{array}$ & Average & \%RSD & Blank \\
\hline $\mathrm{NO}_{3}^{-}$(IC) & $\mathrm{M}$ & $3.04 \mathrm{E}-01$ & $2.91 \mathrm{E}-01$ & 2.97E-01 & $2.81 \mathrm{E}-01$ & 2.93E-01 & $3.2 \%$ & $<3.3 \mathrm{E}-03$ \\
\hline $\mathrm{NO}_{2}^{-}$(IC) & M & 4.92E-01 & 4.97E-01 & 5.10E-01 & 4.77E-01 & 4.94E-01 & $2.7 \%$ & $<4.4 \mathrm{E}-03$ \\
\hline $\mathrm{PO}_{4}^{3-}$ (IC) & $\mathrm{M}$ & $<2.2 \mathrm{E}-03$ & $<2.1 \mathrm{E}-03$ & $<2.1 \mathrm{E}-03$ & $<2.1 \mathrm{E}-03$ & - & - & $<2.1 \mathrm{E}-03$ \\
\hline $\mathrm{SO}_{4}^{2-}(\mathrm{IC})$ & $\mathrm{M}$ & $2.64 \mathrm{E}-02$ & $2.61 \mathrm{E}-02$ & 2.67E-02 & $2.52 \mathrm{E}-02$ & 2.61E-02 & $2.5 \%$ & $<1.1 \mathrm{E}-03$ \\
\hline $\mathrm{C}_{2} \mathrm{O}_{4}{ }^{2-}$ (IC) & M & $3.27 \mathrm{E}-03$ & $3.45 \mathrm{E}-03$ & 3.39E-03 & $3.20 \mathrm{E}-03$ & 3.33E-03 & $3.3 \%$ & $<2.3 \mathrm{E}-03$ \\
\hline $\mathrm{Cl}^{-}$(IC) & $\mathrm{M}$ & $1.16 \mathrm{E}-03$ & $1.14 \mathrm{E}-03$ & $1.12 \mathrm{E}-03$ & $1.14 \mathrm{E}-03$ & $1.14 \mathrm{E}-03$ & $1.4 \%$ & $<1.1 \mathrm{E}-03$ \\
\hline $\mathrm{F}^{-}(\mathrm{IC})$ & M & 2.17E-03 & 2.13E-03 & 2.09E-03 & 2.12E-03 & 2.13E-03 & $1.4 \%$ & $<2.1 \mathrm{E}-03$ \\
\hline $\mathrm{CHO}_{2}^{-}$(IC) & $\mathrm{M}$ & $<4.6 \mathrm{E}-03$ & $<4.5 \mathrm{E}-03$ & $<4.4 \mathrm{E}-03$ & $<4.5 \mathrm{E}-03$ & - & - & $<4.5 \mathrm{E}-03$ \\
\hline $\mathrm{OH}_{\text {free }}^{-}(\mathrm{T})$ & M & $2.05 \mathrm{E}-02$ & $3.64 \mathrm{E}-02$ & $<2.0 \mathrm{E}-02$ & 3.89E-02 & 3.19E-02* & $31 \%$ & $<2.0 \mathrm{E}-02$ \\
\hline Ag (IE) & $\mathrm{mg} / \mathrm{L}$ & $<1.2 \mathrm{E}+01$ & $<1.2 \mathrm{E}+01$ & $<1.2 \mathrm{E}+01$ & $<1.2 \mathrm{E}+01$ & - & - & $<1.2 \mathrm{E}+01$ \\
\hline Al (IE) & $\mathrm{mg} / \mathrm{L}$ & $3.62 \mathrm{E}+02$ & $3.71 \mathrm{E}+02$ & $2.35 \mathrm{E}+02^{\mathrm{a}}$ & $3.47 \mathrm{E}+02$ & $3.60 \mathrm{E}+02 *$ & $3.3 \%$ & $<4.2 \mathrm{E}+01$ \\
\hline B (IE) & $\mathrm{mg} / \mathrm{L}$ & $<1.0 \mathrm{E}+01$ & $<1.0 \mathrm{E}+01$ & $<1.0 \mathrm{E}+01$ & $<1.0 \mathrm{E}+01$ & - & - & $<1.0 \mathrm{E}+01$ \\
\hline Ba (IE) & $\mathrm{mg} / \mathrm{L}$ & $<2.6 \mathrm{E}+00$ & $<2.6 \mathrm{E}+00$ & $<2.6 \mathrm{E}+00$ & $<2.5 \mathrm{E}+00$ & - & - & $<2.6 \mathrm{E}+00$ \\
\hline Be (IE) & $\mathrm{mg} / \mathrm{L}$ & $<2.6 \mathrm{E}-01$ & $<2.6 \mathrm{E}-01$ & $<2.6 \mathrm{E}-01$ & $<2.6 \mathrm{E}-01$ & - & - & $<2.6 \mathrm{E}-01$ \\
\hline Ca (IE) & $\mathrm{mg} / \mathrm{L}$ & $<4.0 \mathrm{E}+01$ & $<4.0 \mathrm{E}+01$ & $<4.0 \mathrm{E}+01$ & $<4.0 \mathrm{E}+01$ & - & - & $<4.0 \mathrm{E}+01$ \\
\hline Cd (IE) & $\mathrm{mg} / \mathrm{L}$ & $<3.4 \mathrm{E}+00$ & $<3.3 \mathrm{E}+00$ & $<3.3 \mathrm{E}+00$ & $<3.3 \mathrm{E}+00$ & - & - & $<3.3 \mathrm{E}+00$ \\
\hline Ce (IE) & $\mathrm{mg} / \mathrm{L}$ & $<2.3 \mathrm{E}+02$ & $<2.3 \mathrm{E}+02$ & $<2.3 \mathrm{E}+02$ & $<2.3 \mathrm{E}+02$ & - & - & $<2.3 \mathrm{E}+02$ \\
\hline Cr (IE) & $\mathrm{mg} / \mathrm{L}$ & $3.64 \mathrm{E}+01$ & $3.77 \mathrm{E}+01$ & $2.39 \mathrm{E}+01^{\mathrm{a}}$ & $3.49 \mathrm{E}+01$ & $3.63 \mathrm{E}+01^{*}$ & $3.8 \%$ & $<4.2 \mathrm{E}+00$ \\
\hline $\mathrm{Cu}$ (IE) & $\mathrm{mg} / \mathrm{L}$ & $<6.7 \mathrm{E}+00$ & $<6.7 \mathrm{E}+00$ & $<6.7 \mathrm{E}+00$ & $<6.7 \mathrm{E}+00$ & - & - & $<6.7 \mathrm{E}+00$ \\
\hline $\mathrm{Fe}$ (IE) & $\mathrm{mg} / \mathrm{L}$ & $<6.5 \mathrm{E}+00$ & $<6.5 \mathrm{E}+00$ & $<7.6 \mathrm{E}+00$ & $<6.5 \mathrm{E}+00$ & - & - & $<6.5 \mathrm{E}+00$ \\
\hline
\end{tabular}

* - Indicate that not all replicates were used to calculate the Average and the \%RSD.

a - Result not included in the calculation of Average and \%RSD since it was more than $30 \%$ outside the mean of the other replicates. 
WSRC-STI-2007-00697, REV. 0

Table 3-3. Composition of the As-Received Tank 51H Filtered Supernate (Continued)

\begin{tabular}{|c|c|c|c|c|c|c|c|c|}
\hline Analyte (Method) & Units & $\begin{array}{c}\text { 1st } \\
\text { Replicate } \\
\end{array}$ & $\begin{array}{c}\text { 2nd } \\
\text { Replicate }\end{array}$ & $\begin{array}{c}\text { 3rd } \\
\text { Replicate }\end{array}$ & $\begin{array}{c}\text { 4th } \\
\text { Replicate }\end{array}$ & Average & \%RSD & Blank \\
\hline Gd (IE) & $\mathrm{mg} / \mathrm{L}$ & $<1.4 \mathrm{E}+01$ & $<1.4 \mathrm{E}+01$ & $<1.4 \mathrm{E}+01$ & $<1.4 \mathrm{E}+01$ & - & - & $<1.4 \mathrm{E}+01$ \\
\hline $\mathrm{K}$ (IE) & $\mathrm{mg} / \mathrm{L}$ & $<2.0 \mathrm{E}+02$ & $<2.0 \mathrm{E}+02$ & $<2.0 \mathrm{E}+02$ & $<1.9 \mathrm{E}+02$ & - & - & $<1.9 \mathrm{E}+02$ \\
\hline La (IE) & $\mathrm{mg} / \mathrm{L}$ & $<1.7 \mathrm{E}+01$ & $<1.7 \mathrm{E}+01$ & $<1.7 \mathrm{E}+01$ & $<1.7 \mathrm{E}+01$ & - & - & $<1.7 \mathrm{E}+01$ \\
\hline Li (IE) & $\mathrm{mg} / \mathrm{L}$ & $<1.2 \mathrm{E}+01$ & $<1.2 \mathrm{E}+01$ & $<1.2 \mathrm{E}+01$ & $<1.2 \mathrm{E}+01$ & - & - & $<1.2 \mathrm{E}+01$ \\
\hline $\mathrm{Mg}$ (IE) & $\mathrm{mg} / \mathrm{L}$ & $<1.4 \mathrm{E}+00$ & $<1.4 \mathrm{E}+00$ & $<1.4 \mathrm{E}+00$ & $<1.4 \mathrm{E}+00$ & - & - & $<1.4 \mathrm{E}+00$ \\
\hline Mn (IE) & $\mathrm{mg} / \mathrm{L}$ & $<9.0 \mathrm{E}-01$ & $<9.0 \mathrm{E}-01$ & $<9.0 \mathrm{E}-01$ & $<8.9 \mathrm{E}-01$ & - & - & $<9.0 \mathrm{E}-01$ \\
\hline Mo (IE) & $\mathrm{mg} / \mathrm{L}$ & $<1.3 \mathrm{E}+01$ & $<1.3 \mathrm{E}+01$ & $<1.3 \mathrm{E}+01$ & $<1.3 \mathrm{E}+01$ & - & - & $<1.3 \mathrm{E}+01$ \\
\hline $\mathrm{Na}$ (IE) & $\mathrm{mg} / \mathrm{L}$ & $2.41 \mathrm{E}+04$ & $2.47 \mathrm{E}+04$ & $1.63 \mathrm{E}+04^{\mathrm{a}}$ & $2.34 \mathrm{E}+04$ & $2.41 \mathrm{E}+04^{*}$ & $2.6 \%$ & $<1.1 \mathrm{E}+02$ \\
\hline $\mathrm{Ni}$ (IE) & $\mathrm{mg} / \mathrm{L}$ & $<9.4 \mathrm{E}+00$ & $<9.4 \mathrm{E}+00$ & $<9.4 \mathrm{E}+00$ & $<9.3 \mathrm{E}+00$ & - & - & $<9.3 \mathrm{E}+00$ \\
\hline $\mathrm{P}$ (IE) & $\mathrm{mg} / \mathrm{L}$ & $<6.0 \mathrm{E}+01$ & $<6.0 \mathrm{E}+01$ & $<6.0 \mathrm{E}+01$ & $<5.9 \mathrm{E}+01$ & - & - & $<6.0 \mathrm{E}+01$ \\
\hline $\mathrm{Pb}$ (IE) & $\mathrm{mg} / \mathrm{L}$ & $<3.4 \mathrm{E}+01$ & $<3.4 \mathrm{E}+01$ & $<3.4 \mathrm{E}+01$ & $<3.3 \mathrm{E}+01$ & - & - & $<3.4 \mathrm{E}+01$ \\
\hline$S$ (IE) & $\mathrm{mg} / \mathrm{L}$ & $<7.9 \mathrm{E}+02$ & $<8.7 \mathrm{E}+02$ & $<7.9 \mathrm{E}+02$ & $<7.8 \mathrm{E}+02$ & - & - & $<7.9 \mathrm{E}+02$ \\
\hline Sb (IE) & $\mathrm{mg} / \mathrm{L}$ & $<6.5 \mathrm{E}+01$ & $<6.4 \mathrm{E}+01$ & $<6.4 \mathrm{E}+01$ & $<6.4 \mathrm{E}+01$ & - & - & $<6.4 \mathrm{E}+01$ \\
\hline Si (IE) & $\mathrm{mg} / \mathrm{L}$ & $<1.1 \mathrm{E}+02$ & $<1.1 \mathrm{E}+02$ & $<1.1 \mathrm{E}+02$ & $<1.1 \mathrm{E}+02$ & - & - & $<1.1 \mathrm{E}+02$ \\
\hline Sn (IE) & $\mathrm{mg} / \mathrm{L}$ & $<1.9 \mathrm{E}+02$ & $<1.9 \mathrm{E}+02$ & $<1.9 \mathrm{E}+02$ & $<1.9 \mathrm{E}+02$ & - & - & $<1.9 \mathrm{E}+02$ \\
\hline Sr (IE) & $\mathrm{mg} / \mathrm{L}$ & $<1.7 \mathrm{E}+01$ & $<1.7 \mathrm{E}+01$ & $<1.7 \mathrm{E}+01$ & $<1.7 \mathrm{E}+01$ & - & - & $<1.7 \mathrm{E}+01$ \\
\hline Ti (IE) & $\mathrm{mg} / \mathrm{L}$ & $<3.8 \mathrm{E}+00$ & $<3.8 \mathrm{E}+00$ & $<3.8 \mathrm{E}+00$ & $<3.8 \mathrm{E}+00$ & - & - & $<3.8 \mathrm{E}+00$ \\
\hline U (IE) & $\mathrm{mg} / \mathrm{L}$ & $<7.8 \mathrm{E}+02$ & $<7.8 \mathrm{E}+02$ & $<7.8 \mathrm{E}+02$ & $<7.7 \mathrm{E}+02$ & - & - & $<7.8 \mathrm{E}+02$ \\
\hline V (IE) & $\mathrm{mg} / \mathrm{L}$ & $<3.2 \mathrm{E}+00$ & $<3.2 \mathrm{E}+00$ & $<3.2 \mathrm{E}+00$ & $<3.2 \mathrm{E}+00$ & - & - & $<3.2 \mathrm{E}+00$ \\
\hline $\mathrm{Zn}$ (IE) & $\mathrm{mg} / \mathrm{L}$ & $<4.2 \mathrm{E}+01$ & $<4.2 \mathrm{E}+01$ & $<4.2 \mathrm{E}+01$ & $<4.2 \mathrm{E}+01$ & - & - & $<4.2 \mathrm{E}+01$ \\
\hline Zr (IE) & $\mathrm{mg} / \mathrm{L}$ & $<4.6 \mathrm{E}+00$ & $<4.6 \mathrm{E}+00$ & $<4.6 \mathrm{E}+00$ & $<4.6 \mathrm{E}+00$ & - & - & $<4.6 \mathrm{E}+00$ \\
\hline
\end{tabular}

* - Indicate that not all replicates were used to calculate the Average and the \%RSD.

a - Result not included in the calculation of Average and \%RSD since it was more than $30 \%$ outside the mean of the other replicates. 
WSRC-STI-2007-00697, REV. 0

Table 3-3. Composition of the As-Received Tank 51H Filtered Supernate (Continued)

\begin{tabular}{|c|c|c|c|c|c|c|c|c|}
\hline Analyte (Method) & Units & $\begin{array}{c}\text { 1st } \\
\text { Replicate }\end{array}$ & $\begin{array}{c}\text { 2nd } \\
\text { Replicate }\end{array}$ & $\begin{array}{c}\text { 3rd } \\
\text { Replicate }\end{array}$ & $\begin{array}{c}\text { 4th } \\
\text { Replicate }\end{array}$ & Average & \%RSD & Blank \\
\hline $\mathrm{Hg}(\mathrm{CV})$ & $\mathrm{mg} / \mathrm{L}$ & $2.43 \mathrm{E}+01$ & $2.30 \mathrm{E}+01$ & $1.91 \mathrm{E}+01$ & $2.24 \mathrm{E}+01$ & $2.33 E+01$ & $9.5 \%$ & $<2.2 \mathrm{E}+00$ \\
\hline${ }^{233} \mathrm{U}(\mathrm{IM})$ & $\mathrm{mg} / \mathrm{L}$ & $<8.2 \mathrm{E}-02$ & $<8.2 \mathrm{E}-02$ & $<8.2 \mathrm{E}-02$ & $<8.1 \mathrm{E}-02$ & - & - & $<8.1 \mathrm{E}-02$ \\
\hline${ }^{234} \mathrm{U}(\mathrm{IM})$ & $\mathrm{mg} / \mathrm{L}$ & $<2.1 \mathrm{E}-02$ & $<2.1 \mathrm{E}-02$ & $<2.1 \mathrm{E}-02$ & $<2.1 \mathrm{E}-02$ & - & - & $<2.1 \mathrm{E}-02$ \\
\hline${ }^{235} \mathrm{U}(\mathrm{IM})$ & $\mathrm{mg} / \mathrm{L}$ & $<3.0 \mathrm{E}-02$ & $<3.0 \mathrm{E}-02$ & $<3.0 \mathrm{E}-02$ & $<2.9 \mathrm{E}-02$ & - & - & $<3.0 \mathrm{E}-02$ \\
\hline${ }^{236} \mathrm{U}$ (IM) & $\mathrm{mg} / \mathrm{L}$ & $<1.3 \mathrm{E}-02$ & $<1.3 \mathrm{E}-02$ & $<1.3 \mathrm{E}-02$ & $<1.3 \mathrm{E}-02$ & - & - & $<1.3 \mathrm{E}-02$ \\
\hline${ }^{238} \mathrm{U}$ (IM) & $\mathrm{mg} / \mathrm{L}$ & $1.49 \mathrm{E}-01$ & 1.39E-01 & $1.47 \mathrm{E}-01$ & $1.49 \mathrm{E}-01$ & $1.49 \mathrm{E}-01$ & $0.8 \%$ & $<8.1 \mathrm{E}-02$ \\
\hline${ }^{99}$ Tc (IM) & $\mu \mathrm{Ci} / \mathrm{mL}$ & 9.64E-03 & 8.92E-03 & 8.89E-03 & 8.77E-03 & $9.06 \mathrm{E}-03$ & $4.4 \%$ & $<7.1 \mathrm{E}-04$ \\
\hline${ }^{137} \mathrm{Cs}$ (IM) & $\mu \mathrm{Ci} / \mathrm{mL}$ & $2.34 \mathrm{E}+01$ & $2.28 \mathrm{E}+01$ & $2.18 \mathrm{E}+01$ & $2.03 \mathrm{E}+01$ & $2.21 \mathrm{E}+01$ & $6.1 \%$ & $<7.9 \mathrm{E}-01$ \\
\hline${ }^{237} \mathrm{~Np}$ (IM) & $\mu \mathrm{Ci} / \mathrm{mL}$ & $<5.1 \mathrm{E}-05$ & $<5.1 \mathrm{E}-05$ & $<5.1 \mathrm{E}-05$ & $<5.1 \mathrm{E}-05$ & - & - & $<5.1 \mathrm{E}-05$ \\
\hline${ }^{239} \mathrm{Pu}$ (IM) & $\mu \mathrm{Ci} / \mathrm{mL}$ & $<1.0 \mathrm{E}-03$ & $<1.0 \mathrm{E}-03$ & $<1.0 \mathrm{E}-03$ & $<9.9 \mathrm{E}-04$ & - & - & $<1.0 \mathrm{E}-03$ \\
\hline${ }^{240} \mathrm{Pu}$ (IM) & $\mu \mathrm{Ci} / \mathrm{mL}$ & $<2.0 \mathrm{E}-02$ & $<2.0 \mathrm{E}-02$ & $<2.0 \mathrm{E}-02$ & $<2.0 \mathrm{E}-02$ & - & - & $<2.0 \mathrm{E}-02$ \\
\hline${ }^{238} \mathrm{Pu}(\mathrm{SA})$ & $\mu \mathrm{Ci} / \mathrm{mL}$ & 1.19E-02 & $1.22 \mathrm{E}-02$ & $1.07 \mathrm{E}-02$ & $1.18 \mathrm{E}-02$ & 1.16E-02 & $5.8 \%$ & $1.20 \mathrm{E}-04$ \\
\hline${ }^{239 / 240} \mathrm{Pu}(\mathrm{SA})$ & $\mu \mathrm{Ci} / \mathrm{mL}$ & $5.60 \mathrm{E}-04$ & $6.23 \mathrm{E}-04$ & 6.03E-04 & 6.91E-04 & 6.19E-04 & $8.9 \%$ & $1.10 \mathrm{E}-04$ \\
\hline${ }^{241} \mathrm{Pu}$ (SA) & $\mu \mathrm{Ci} / \mathrm{mL}$ & 7.94E-03 & 8.79E-03 & $5.48 \mathrm{E}-03$ & $<7.2 \mathrm{E}-03$ & $7.40 \mathrm{E}-03^{*}$ & $23 \%$ & $<4.0 \mathrm{E}-03$ \\
\hline
\end{tabular}

* - Indicate that not all replicates were used to calculate the Average and the \%RSD. 
WSRC-STI-2007-00697, REV. 0

Table 3-4. Composition of the Aqua Regia Dissolution of Total Dried Solids from As-Received Tank 51H Sludge

\begin{tabular}{|c|c|c|c|c|c|c|c|c|c|c|}
\hline Analyte (Method) & Units & $\begin{array}{c}\text { 1st } \\
\text { Replicate } \\
\end{array}$ & $\begin{array}{c}\text { 2nd } \\
\text { Replicate } \\
\end{array}$ & $\begin{array}{c}\text { 3rd } \\
\text { Replicate } \\
\end{array}$ & $\begin{array}{c}\text { 4th } \\
\text { Replicate } \\
\end{array}$ & Average & \%RSD & Blank & $\begin{array}{c}\text { Average } \\
\text { Analyzed } \\
\text { Glass Std }\end{array}$ & $\begin{array}{c}\text { Glass Std } \\
\text { Composition }\end{array}$ \\
\hline Ag (IE) & $\mathrm{mg} / \mathrm{kg}$ & $<2.6 \mathrm{E}+02$ & $<2.6 \mathrm{E}+02$ & $<2.6 \mathrm{E}+02$ & $<2.6 \mathrm{E}+02$ & - & - & $<2.9 \mathrm{E}-01$ & $<3.0 \mathrm{E}+02$ & - \\
\hline $\mathrm{Al}$ (IE) & $\mathrm{mg} / \mathrm{kg}$ & $5.72 \mathrm{E}+04$ & $9.13 E+04$ & $8.24 \mathrm{E}+04$ & $7.94 \mathrm{E}+04$ & $7.76 E+04$ & $19 \%$ & $<1.0 \mathrm{E}+00$ & $2.41 \mathrm{E}+04$ & $2.50 \mathrm{E}+04$ \\
\hline B (IE) & $\mathrm{mg} / \mathrm{kg}$ & $<2.2 \mathrm{E}+02$ & $<2.2 \mathrm{E}+02$ & $<2.2 \mathrm{E}+02$ & $<2.3 \mathrm{E}+02$ & - & - & $<2.5 \mathrm{E}-01$ & $2.53 \mathrm{E}+04$ & $2.69 \mathrm{E}+04$ \\
\hline $\mathrm{Ba}$ (IE) & $\mathrm{mg} / \mathrm{kg}$ & $4.22 \mathrm{E}+02$ & $4.51 \mathrm{E}+02$ & $4.58 \mathrm{E}+02$ & $4.51 E+02$ & $4.46 E+02$ & $3.6 \%$ & $<6.3 \mathrm{E}-02$ & $8.08 \mathrm{E}+02$ & $7.90 \mathrm{E}+02$ \\
\hline Be (IE) & $\mathrm{mg} / \mathrm{kg}$ & $<7.6 \mathrm{E}+00$ & $<7.5 \mathrm{E}+00$ & $<7.5 \mathrm{E}+00$ & $<7.7 \mathrm{E}+00$ & - & - & $<8.5 \mathrm{E}-03$ & $2.07 \mathrm{E}+01$ & - \\
\hline Ca (IE) & $\mathrm{mg} / \mathrm{kg}$ & $7.42 \mathrm{E}+03$ & $7.92 \mathrm{E}+03$ & $7.91 \mathrm{E}+03$ & $7.83 E+03$ & $7.77 E+03$ & $3.0 \%$ & $<9.8 \mathrm{E}-01$ & $1.04 \mathrm{E}+04$ & $1.02 \mathrm{E}+04$ \\
\hline Cd (IE) & $\mathrm{mg} / \mathrm{kg}$ & $2.60 \mathrm{E}+02$ & $2.85 E+02$ & $2.84 \mathrm{E}+02$ & $2.81 E+02$ & $2.78 \mathrm{E}+02$ & $4.2 \%$ & $<8.2 \mathrm{E}-02$ & $<8.4 \mathrm{E}+01$ & - \\
\hline Ce (IE) & $\mathrm{mg} / \mathrm{kg}$ & $<5.0 \mathrm{E}+03$ & $<5.0 \mathrm{E}+03$ & $<5.0 \mathrm{E}+03$ & $<5.1 \mathrm{E}+03$ & - & - & $<5.7 \mathrm{E}+00$ & $<5.8 \mathrm{E}+03$ & - \\
\hline Cr (IE) & $\mathrm{mg} / \mathrm{kg}$ & $4.49 \mathrm{E}+02$ & $4.69 \mathrm{E}+02$ & $4.57 \mathrm{E}+02$ & $4.79 E+02$ & $4.64 \mathrm{E}+02$ & $2.8 \%$ & $<1.0 \mathrm{E}-01$ & $6.86 \mathrm{E}+02$ & $6.40 \mathrm{E}+02$ \\
\hline $\mathrm{Cu}$ (IE) & $\mathrm{mg} / \mathrm{kg}$ & $3.03 E+02$ & $3.35 E+02$ & $3.31 \mathrm{E}+02$ & $3.21 E+02$ & $3.23 E+02$ & $4.4 \%$ & $<1.7 \mathrm{E}-01$ & $<1.7 \mathrm{E}+02$ & $3.00 \mathrm{E}+01$ \\
\hline Fe (IE) & $\mathrm{mg} / \mathrm{kg}$ & $6.87 \mathrm{E}+04$ & $7.32 \mathrm{E}+04$ & $7.24 \mathrm{E}+04$ & $7.27 \mathrm{E}+04$ & $7.18 \mathrm{E}+04$ & $2.9 \%$ & $<1.6 \mathrm{E}-01$ & $9.69 \mathrm{E}+04$ & $9.79 \mathrm{E}+04$ \\
\hline Gd (IE) & $\mathrm{mg} / \mathrm{kg}$ & $<3.1 \mathrm{E}+02$ & $<3.1 \mathrm{E}+02$ & $<3.1 \mathrm{E}+02$ & $<3.1 \mathrm{E}+02$ & - & - & $<3.5 \mathrm{E}-01$ & $<3.5 \mathrm{E}+02$ & - \\
\hline K (IE) & $\mathrm{mg} / \mathrm{kg}$ & $<4.3 \mathrm{E}+03$ & $<4.2 \mathrm{E}+03$ & $<4.2 \mathrm{E}+03$ & $<4.3 \mathrm{E}+03$ & - & - & $<4.8 \mathrm{E}+00$ & $2.11 \mathrm{E}+04$ & $2.26 \mathrm{E}+04$ \\
\hline La (IE) & $\mathrm{mg} / \mathrm{kg}$ & $<3.8 \mathrm{E}+02$ & $<3.7 \mathrm{E}+02$ & $<3.7 \mathrm{E}+02$ & $<3.8 \mathrm{E}+02$ & - & - & $<4.3 \mathrm{E}-01$ & $<4.3 \mathrm{E}+02$ & - \\
\hline Li (IE) & $\mathrm{mg} / \mathrm{kg}$ & $<2.6 \mathrm{E}+02$ & $<2.6 \mathrm{E}+02$ & $<2.6 \mathrm{E}+02$ & $<2.7 \mathrm{E}+02$ & - & - & $<2.9 \mathrm{E}-01$ & $1.48 \mathrm{E}+04$ & $1.49 \mathrm{E}+04$ \\
\hline Mg (IE) & $\mathrm{mg} / \mathrm{kg}$ & $2.90 \mathrm{E}+03$ & $3.09 E+03$ & $3.04 \mathrm{E}+03$ & $3.10 \mathrm{E}+03$ & $3.03 E+03$ & $3.0 \%$ & $3.11 \mathrm{E}-01$ & $5.15 \mathrm{E}+03$ & $5.20 \mathrm{E}+03$ \\
\hline $\mathrm{Mn}$ (IE) & $\mathrm{mg} / \mathrm{kg}$ & $1.67 \mathrm{E}+04$ & $1.78 \mathrm{E}+04$ & $1.77 \mathrm{E}+04$ & $1.79 \mathrm{E}+04$ & $1.75 E+04$ & $3.2 \%$ & $<2.2 \mathrm{E}-02$ & $1.42 \mathrm{E}+04$ & $1.46 \mathrm{E}+04$ \\
\hline Mo (IE) & $\mathrm{mg} / \mathrm{kg}$ & $<2.8 \mathrm{E}+02$ & $<2.8 \mathrm{E}+02$ & $<2.8 \mathrm{E}+02$ & $<2.9 \mathrm{E}+02$ & - & - & $<3.2 \mathrm{E}-01$ & $<3.2 \mathrm{E}+02$ & - \\
\hline $\mathrm{Na}$ (IE) & $\mathrm{mg} / \mathrm{kg}$ & $1.38 \mathrm{E}+05$ & $1.48 \mathrm{E}+05$ & $1.47 \mathrm{E}+05$ & $1.48 \mathrm{E}+05$ & $1.45 E+05$ & $3.3 \%$ & $<2.8 \mathrm{E}+00$ & $8.29 \mathrm{E}+04$ & $8.52 \mathrm{E}+04$ \\
\hline $\mathrm{Ni}$ (IE) & $\mathrm{mg} / \mathrm{kg}$ & $6.15 \mathrm{E}+03$ & $6.56 \mathrm{E}+03$ & $6.50 \mathrm{E}+03$ & $6.53 E+03$ & $6.44 E+03$ & $3.0 \%$ & $<2.3 \mathrm{E}-01$ & $8.17 \mathrm{E}+03$ & $8.27 \mathrm{E}+03$ \\
\hline
\end{tabular}

Divide $\mathrm{mg} / \mathrm{kg}$ values by $1 \mathrm{E}+04$ to convert to wt \% dried solids basis 
WSRC-STI-2007-00697, REV. 0

Table 3-4. Composition of the Aqua Regia Dissolution of Total Dried Solids from As-Received Tank 51H Sludge (Continued)

\begin{tabular}{|c|c|c|c|c|c|c|c|c|c|c|}
\hline Analyte (Method) & Units & $\begin{array}{c}\text { 1st } \\
\text { Replicate } \\
\end{array}$ & $\begin{array}{c}\text { 2nd } \\
\text { Replicate } \\
\end{array}$ & $\begin{array}{c}\text { 3rd } \\
\text { Replicate } \\
\end{array}$ & $\begin{array}{c}\text { 4th } \\
\text { Replicate } \\
\end{array}$ & Average & \%RSD & Blank & $\begin{array}{c}\text { Average } \\
\text { Analyzed } \\
\text { Glass Std }\end{array}$ & $\begin{array}{c}\text { Glass Std } \\
\text { Composition }\end{array}$ \\
\hline $\mathrm{P}$ (IE) & $\mathrm{mg} / \mathrm{kg}$ & $<1.3 \mathrm{E}+03$ & $<1.3 \mathrm{E}+03$ & $<1.3 \mathrm{E}+03$ & $<1.3 \mathrm{E}+03$ & - & - & $<1.5 \mathrm{E}+00$ & $<1.5 \mathrm{E}+03$ & $1.10 \mathrm{E}+03$ \\
\hline $\mathrm{Pb}$ (IE) & $\mathrm{mg} / \mathrm{kg}$ & $<7.3 \mathrm{E}+02$ & $<7.3 \mathrm{E}+02$ & $<7.2 \mathrm{E}+02$ & $<7.4 \mathrm{E}+02$ & - & - & $<8.3 \mathrm{E}-01$ & $<8.4 \mathrm{E}+02$ & - \\
\hline S (IE) & $\mathrm{mg} / \mathrm{kg}$ & $<1.7 \mathrm{E}+04$ & $<1.7 \mathrm{E}+04$ & $<1.7 \mathrm{E}+04$ & $<1.7 \mathrm{E}+04$ & - & - & $<1.9 \mathrm{E}+01$ & $<2.0 \mathrm{E}+04$ & - \\
\hline Sb (IE) & $\mathrm{mg} / \mathrm{kg}$ & $<1.4 \mathrm{E}+03$ & $<1.4 \mathrm{E}+03$ & $<1.4 \mathrm{E}+03$ & $<1.4 \mathrm{E}+03$ & - & - & $<1.6 \mathrm{E}+00$ & $<1.6 \mathrm{E}+03$ & - \\
\hline Sn (IE) & $\mathrm{mg} / \mathrm{kg}$ & $<4.2 \mathrm{E}+03$ & $<4.1 \mathrm{E}+03$ & $<4.1 \mathrm{E}+03$ & $<4.2 \mathrm{E}+03$ & - & - & $<4.7 \mathrm{E}+00$ & $<4.8 \mathrm{E}+03$ & - \\
\hline Sr (IE) & $\mathrm{mg} / \mathrm{kg}$ & $<3.7 \mathrm{E}+02$ & $<3.7 \mathrm{E}+02$ & $<3.7 \mathrm{E}+02$ & $<3.8 \mathrm{E}+02$ & - & - & $<4.2 \mathrm{E}-01$ & $<4.3 \mathrm{E}+02$ & $3.00 \mathrm{E}+01$ \\
\hline Ti (IE) & $\mathrm{mg} / \mathrm{kg}$ & $<8.5 \mathrm{E}+01$ & $<8.4 \mathrm{E}+01$ & $<8.4 \mathrm{E}+01$ & $<8.6 \mathrm{E}+01$ & - & - & $<9.3 \mathrm{E}-02$ & $5.94 \mathrm{E}+03$ & $6.90 \mathrm{E}+03$ \\
\hline U (IE) & $\mathrm{mg} / \mathrm{kg}$ & $1.69 \mathrm{E}+04$ & $1.72 \mathrm{E}+04$ & $1.74 \mathrm{E}+04$ & $1.76 \mathrm{E}+04$ & $1.73 E+04$ & $1.7 \%$ & $<1.9 \mathrm{E}+01$ & $<1.9 \mathrm{E}+04$ & - \\
\hline V (IE) & $\mathrm{mg} / \mathrm{kg}$ & $<7.0 \mathrm{E}+01$ & $<6.9 \mathrm{E}+01$ & $<6.9 \mathrm{E}+01$ & $<7.1 \mathrm{E}+01$ & - & - & $<7.9 \mathrm{E}-02$ & $<8.0 \mathrm{E}+01$ & - \\
\hline Zn (IE) & $\mathrm{mg} / \mathrm{kg}$ & $<9.1 \mathrm{E}+02$ & $<9.1 \mathrm{E}+02$ & $<9.0 \mathrm{E}+02$ & $<9.2 \mathrm{E}+02$ & - & - & $<1.0 \mathrm{E}+00$ & $<1.0 \mathrm{E}+03$ & $1.60 \mathrm{E}+02$ \\
\hline $\mathrm{Zr}$ (IE) & $\mathrm{mg} / \mathrm{kg}$ & $2.05 \mathrm{E}+02$ & $2.87 \mathrm{E}+02$ & $1.93 \mathrm{E}+02$ & $2.57 \mathrm{E}+02$ & $2.36 \mathrm{E}+02$ & $19 \%$ & $<1.1 \mathrm{E}-01$ & $6.37 \mathrm{E}+02$ & $9.60 \mathrm{E}+02$ \\
\hline $\mathrm{Hg}(\mathrm{CV})$ & $\mathrm{mg} / \mathrm{kg}$ & $1.73 \mathrm{E}+04$ & $1.72 \mathrm{E}+04$ & $1.77 \mathrm{E}+04$ & $1.68 \mathrm{E}+04$ & $1.73 E+04$ & $2.1 \%$ & $<4.8 \mathrm{E}+01$ & - & - \\
\hline
\end{tabular}

Divide $\mathrm{mg} / \mathrm{kg}$ values by $1 \mathrm{E}+04$ to convert to wt \% dried solids basis 
WSRC-STI-2007-00697, REV. 0

Table 3-5. Composition of the Peroxide Fusion Dissolution of Total Dried Solids from As-Received Tank 51H Sludge

\begin{tabular}{|c|c|c|c|c|c|c|c|c|c|c|}
\hline Analyte (Method) & Units & $\begin{array}{c}\text { 1st } \\
\text { Replicate }\end{array}$ & $\begin{array}{c}\text { 2nd } \\
\text { Replicate }\end{array}$ & $\begin{array}{c}\text { 3rd } \\
\text { Replicate }\end{array}$ & $\begin{array}{c}\text { 4th } \\
\text { Replicate }\end{array}$ & Average & \%RSD & Blank & $\begin{array}{c}\text { Average } \\
\text { Analyzed } \\
\text { Glass Std }\end{array}$ & $\begin{array}{c}\text { Glass Std } \\
\text { Composition }\end{array}$ \\
\hline Ag (IE) & $\mathrm{mg} / \mathrm{kg}$ & $<2.6 \mathrm{E}+02$ & $<2.6 \mathrm{E}+02$ & $<2.6 \mathrm{E}+02$ & $<2.6 \mathrm{E}+02$ & - & - & $<2.9 \mathrm{E}-01$ & $<3.1 \mathrm{E}+02$ & - \\
\hline $\mathrm{Al}$ (IE) & $\mathrm{mg} / \mathrm{kg}$ & $1.60 \mathrm{E}+05$ & $1.61 \mathrm{E}+05$ & $1.59 \mathrm{E}+05$ & $1.43 \mathrm{E}+05$ & $1.56 E+05$ & $5.5 \%$ & $<1.0 \mathrm{E}+00$ & $2.51 \mathrm{E}+04$ & $2.50 \mathrm{E}+04$ \\
\hline B (IE) & $\mathrm{mg} / \mathrm{kg}$ & $<2.2 \mathrm{E}+02$ & $<2.2 \mathrm{E}+02$ & $<2.2 \mathrm{E}+02$ & $<2.2 \mathrm{E}+02$ & - & - & $<2.5 \mathrm{E}-01$ & $2.50 \mathrm{E}+04$ & $2.69 \mathrm{E}+04$ \\
\hline $\mathrm{Ba}$ (IE) & $\mathrm{mg} / \mathrm{kg}$ & $4.56 \mathrm{E}+02$ & $4.67 \mathrm{E}+02$ & $4.55 \mathrm{E}+02$ & $4.54 \mathrm{E}+02$ & $4.58 \mathrm{E}+02$ & $1.3 \%$ & $<6.3 \mathrm{E}-02$ & $7.80 \mathrm{E}+02$ & $7.90 \mathrm{E}+02$ \\
\hline Be (IE) & $\mathrm{mg} / \mathrm{kg}$ & $<7.6 \mathrm{E}+00$ & $<7.6 \mathrm{E}+00$ & $<7.5 \mathrm{E}+00$ & $<7.6 \mathrm{E}+00$ & - & - & $<8.5 \mathrm{E}-03$ & $2.32 \mathrm{E}+01$ & - \\
\hline $\mathrm{Ca}$ (IE) & $\mathrm{mg} / \mathrm{kg}$ & $8.19 E+03$ & $9.17 \mathrm{E}+03$ & $8.15 E+03$ & $8.16 \mathrm{E}+03$ & $8.42 E+03$ & $6.0 \%$ & $1.62 \mathrm{E}+00$ & $1.10 \mathrm{E}+04$ & $1.02 \mathrm{E}+04$ \\
\hline $\mathrm{Cd}$ (IE) & $\mathrm{mg} / \mathrm{kg}$ & $2.69 \mathrm{E}+02$ & $2.78 \mathrm{E}+02$ & $2.52 \mathrm{E}+02$ & $2.59 \mathrm{E}+02$ & $2.65 E+02$ & $4.3 \%$ & $<8.2 \mathrm{E}-02$ & $<8.8 \mathrm{E}+01$ & - \\
\hline Ce (IE) & $\mathrm{mg} / \mathrm{kg}$ & $<5.0 \mathrm{E}+03$ & $<5.0 \mathrm{E}+03$ & $<5.0 \mathrm{E}+03$ & $<5.1 \mathrm{E}+03$ & - & - & $<5.7 \mathrm{E}+00$ & $<6.1 \mathrm{E}+03$ & - \\
\hline Cr (IE) & $\mathrm{mg} / \mathrm{kg}$ & $4.59 \mathrm{E}+02$ & $4.54 \mathrm{E}+02$ & $4.60 \mathrm{E}+02$ & $3.85 \mathrm{E}+02$ & $4.40 \mathrm{E}+02$ & $8.3 \%$ & $<1.0 \mathrm{E}-01$ & $6.79 \mathrm{E}+02$ & $6.40 \mathrm{E}+02$ \\
\hline $\mathrm{Cu}$ (IE) & $\mathrm{mg} / \mathrm{kg}$ & $3.47 \mathrm{E}+02$ & $3.61 \mathrm{E}+02$ & $3.50 \mathrm{E}+02$ & $3.35 E+02$ & $3.48 \mathrm{E}+02$ & $3.1 \%$ & $<1.7 \mathrm{E}-01$ & $<1.8 \mathrm{E}+02$ & $3.00 \mathrm{E}+01$ \\
\hline Fe (IE) & $\mathrm{mg} / \mathrm{kg}$ & $7.48 \mathrm{E}+04$ & $7.48 \mathrm{E}+04$ & $7.43 \mathrm{E}+04$ & $7.10 \mathrm{E}+04$ & $7.37 E+04$ & $2.5 \%$ & $2.71 \mathrm{E}-01$ & $9.51 \mathrm{E}+04$ & $9.79 \mathrm{E}+04$ \\
\hline Gd (IE) & $\mathrm{mg} / \mathrm{kg}$ & $<3.1 \mathrm{E}+02$ & $<3.1 \mathrm{E}+02$ & $<3.1 \mathrm{E}+02$ & $<3.1 \mathrm{E}+02$ & - & - & $<3.5 \mathrm{E}-01$ & $<3.7 \mathrm{E}+02$ & - \\
\hline K (IE) & $\mathrm{mg} / \mathrm{kg}$ & $<4.3 \mathrm{E}+03$ & $<4.3 \mathrm{E}+03$ & $<4.2 \mathrm{E}+03$ & $<4.3 \mathrm{E}+03$ & - & - & $<4.8 \mathrm{E}+00$ & $2.51 \mathrm{E}+04$ & $2.26 \mathrm{E}+04$ \\
\hline La (IE) & $\mathrm{mg} / \mathrm{kg}$ & $<3.8 \mathrm{E}+02$ & $<3.8 \mathrm{E}+02$ & $<3.7 \mathrm{E}+02$ & $<3.8 \mathrm{E}+02$ & - & - & $<4.3 \mathrm{E}-01$ & $<4.5 \mathrm{E}+02$ & - \\
\hline Li (IE) & $\mathrm{mg} / \mathrm{kg}$ & $<2.6 \mathrm{E}+02$ & $<2.6 \mathrm{E}+02$ & $<2.6 \mathrm{E}+02$ & $<2.6 \mathrm{E}+02$ & - & - & $<2.9 \mathrm{E}-01$ & $1.50 \mathrm{E}+04$ & $1.49 \mathrm{E}+04$ \\
\hline Mg (IE) & $\mathrm{mg} / \mathrm{kg}$ & $2.90 \mathrm{E}+03$ & $2.95 \mathrm{E}+03$ & $2.89 \mathrm{E}+03$ & $2.73 E+03$ & $2.87 E+03$ & $3.3 \%$ & $1.80 \mathrm{E}-01$ & $4.80 \mathrm{E}+03$ & $5.20 \mathrm{E}+03$ \\
\hline Mn (IE) & $\mathrm{mg} / \mathrm{kg}$ & $1.75 \mathrm{E}+04$ & $1.73 \mathrm{E}+04$ & $1.74 \mathrm{E}+04$ & $1.62 \mathrm{E}+04$ & $1.71 \mathrm{E}+04$ & $3.5 \%$ & $2.25 \mathrm{E}-02$ & $1.32 \mathrm{E}+04$ & $1.46 \mathrm{E}+04$ \\
\hline Mo (IE) & $\mathrm{mg} / \mathrm{kg}$ & $<2.8 \mathrm{E}+02$ & $<2.8 \mathrm{E}+02$ & $<2.8 \mathrm{E}+02$ & $<2.8 \mathrm{E}+02$ & - & - & $<3.2 \mathrm{E}-01$ & $<3.4 \mathrm{E}+02$ & - \\
\hline $\mathrm{Ni}$ (IE) & $\mathrm{mg} / \mathrm{kg}$ & $6.64 \mathrm{E}+03$ & $6.54 \mathrm{E}+03$ & $6.57 \mathrm{E}+03$ & $6.19 E+03$ & $6.49 E+03$ & $3.1 \%$ & $<2.3 \mathrm{E}-01$ & $6.91 \mathrm{E}+03$ & $8.27 \mathrm{E}+03$ \\
\hline
\end{tabular}

Divide $\mathrm{mg} / \mathrm{kg}$ values by $1 \mathrm{E}+04$ to convert to wt \% dried solids basis 
WSRC-STI-2007-00697, REV. 0

Table 3-5. Composition of the Peroxide Fusion Dissolution of Total Dried Solids from As-Received Tank 51H Sludge (Continued)

\begin{tabular}{|c|c|c|c|c|c|c|c|c|c|c|}
\hline Analyte (Method) & Units & $\begin{array}{c}\text { 1st } \\
\text { Replicate } \\
\end{array}$ & $\begin{array}{c}\text { 2nd } \\
\text { Replicate }\end{array}$ & $\begin{array}{c}\text { 3rd } \\
\text { Replicate }\end{array}$ & $\begin{array}{c}\text { 4th } \\
\text { Replicate }\end{array}$ & Average & \%RSD & Blank & $\begin{array}{c}\text { Average } \\
\text { Analyzed } \\
\text { Glass Std }\end{array}$ & $\begin{array}{c}\text { Glass Std } \\
\text { Composition }\end{array}$ \\
\hline $\mathrm{P}$ (IE) & $\mathrm{mg} / \mathrm{kg}$ & $1.43 \mathrm{E}+03$ & $1.17 \mathrm{E}+03$ & $1.30 \mathrm{E}+03$ & $<1.3 \mathrm{E}+03$ & - & - & $<1.5 \mathrm{E}+00$ & $<1.6 \mathrm{E}+03$ & $1.10 \mathrm{E}+03$ \\
\hline $\mathrm{Pb}$ (IE) & $\mathrm{mg} / \mathrm{kg}$ & $<7.3 \mathrm{E}+02$ & $<7.3 \mathrm{E}+02$ & $<7.3 \mathrm{E}+02$ & $<7.3 \mathrm{E}+02$ & - & - & $<8.3 \mathrm{E}-01$ & $<8.8 \mathrm{E}+02$ & - \\
\hline S (IE) & $\mathrm{mg} / \mathrm{kg}$ & $<1.7 \mathrm{E}+04$ & $<1.7 \mathrm{E}+04$ & $<1.7 \mathrm{E}+04$ & $<1.7 \mathrm{E}+04$ & - & - & $<1.9 \mathrm{E}+01$ & $<2.1 \mathrm{E}+04$ & - \\
\hline $\mathrm{Sb}$ (IE) & $\mathrm{mg} / \mathrm{kg}$ & $<1.4 \mathrm{E}+03$ & $<1.4 \mathrm{E}+03$ & $<1.4 \mathrm{E}+03$ & $<1.4 \mathrm{E}+03$ & - & - & $<1.6 \mathrm{E}+00$ & $<1.7 \mathrm{E}+03$ & - \\
\hline Si (IE) & $\mathrm{mg} / \mathrm{kg}$ & $4.41 \mathrm{E}+03$ & $4.47 \mathrm{E}+03$ & $4.37 \mathrm{E}+03$ & $4.16 \mathrm{E}+03$ & $4.35 E+03$ & $3.1 \%$ & $<2.6 \mathrm{E}+00$ & $2.25 \mathrm{E}+05$ & $2.24 \mathrm{E}+05$ \\
\hline Sn (IE) & $\mathrm{mg} / \mathrm{kg}$ & $<4.2 \mathrm{E}+03$ & $<4.2 \mathrm{E}+03$ & $<4.1 \mathrm{E}+03$ & $<4.2 \mathrm{E}+03$ & - & - & $<4.7 \mathrm{E}+00$ & $<5.0 \mathrm{E}+03$ & - \\
\hline Sr (IE) & $\mathrm{mg} / \mathrm{kg}$ & $<3.7 \mathrm{E}+02$ & $<3.7 \mathrm{E}+02$ & $<3.7 \mathrm{E}+02$ & $<3.7 \mathrm{E}+02$ & - & - & $<4.2 \mathrm{E}-01$ & $<4.5 \mathrm{E}+02$ & $3.00 \mathrm{E}+01$ \\
\hline Ti (IE) & $\mathrm{mg} / \mathrm{kg}$ & $<1.7 \mathrm{E}+02$ & $<1.7 \mathrm{E}+02$ & $<1.7 \mathrm{E}+02$ & $<1.7 \mathrm{E}+02$ & - & - & $<9.3 \mathrm{E}-02$ & $6.65 E+03$ & $6.90 \mathrm{E}+03$ \\
\hline U (IE) & $\mathrm{mg} / \mathrm{kg}$ & $<1.7 \mathrm{E}+04$ & $1.75 \mathrm{E}+04$ & $<1.7 \mathrm{E}+04$ & $<1.7 \mathrm{E}+04$ & - & - & $<1.9 \mathrm{E}+01$ & $<2.0 \mathrm{E}+04$ & - \\
\hline $\mathrm{V}$ (IE) & $\mathrm{mg} / \mathrm{kg}$ & $<7.0 \mathrm{E}+01$ & $<7.0 \mathrm{E}+01$ & $<6.9 \mathrm{E}+01$ & $<7.0 \mathrm{E}+01$ & - & - & $<7.9 \mathrm{E}-02$ & $<8.4 \mathrm{E}+01$ & - \\
\hline $\mathrm{Zn}$ (IE) & $\mathrm{mg} / \mathrm{kg}$ & $<9.1 \mathrm{E}+02$ & $<9.1 \mathrm{E}+02$ & $<9.1 \mathrm{E}+02$ & $<9.1 \mathrm{E}+02$ & - & - & $<1.0 \mathrm{E}+00$ & $<1.1 \mathrm{E}+03$ & $1.60 \mathrm{E}+02$ \\
\hline${ }^{233} \mathrm{U}$ (IM) & $\mathrm{mg} / \mathrm{kg}$ & $<6.7 \mathrm{E}+00$ & $<6.7 \mathrm{E}+00$ & $<6.6 \mathrm{E}+00$ & $<6.7 \mathrm{E}+00$ & - & - & - & - & - \\
\hline${ }^{234} \mathrm{U}$ (IM) & $\mathrm{mg} / \mathrm{kg}$ & $6.96 \mathrm{E}+00$ & $1.03 \mathrm{E}+01$ & $7.69 \mathrm{E}+00$ & $6.36 \mathrm{E}+00$ & $7.83 E+00$ & $22 \%$ & - & - & - \\
\hline${ }^{235} \mathrm{U}$ (IM) & $\mathrm{mg} / \mathrm{kg}$ & $1.21 \mathrm{E}+02$ & $1.27 \mathrm{E}+02$ & $1.20 \mathrm{E}+02$ & $1.16 \mathrm{E}+02$ & $1.21 \mathrm{E}+02$ & $3.8 \%$ & - & - & - \\
\hline${ }^{236} \mathrm{U}$ (IM) & $\mathrm{mg} / \mathrm{kg}$ & $9.81 \mathrm{E}+00$ & $1.24 \mathrm{E}+01$ & $1.28 \mathrm{E}+01$ & $1.01 \mathrm{E}+01$ & $1.13 E+01$ & $14 \%$ & - & - & - \\
\hline${ }^{238} \mathrm{U}$ (IM) & $\mathrm{mg} / \mathrm{kg}$ & $1.49 \mathrm{E}+04$ & $1.65 \mathrm{E}+04$ & $1.56 \mathrm{E}+04$ & $1.39 \mathrm{E}+04$ & $1.52 E+04$ & $7.2 \%$ & - & - & - \\
\hline U total (IM) & $\mathrm{mg} / \mathrm{kg}$ & $1.50 \mathrm{E}+04$ & $1.66 \mathrm{E}+04$ & $1.57 \mathrm{E}+04$ & $1.40 \mathrm{E}+04$ & $1.54 E+04$ & $7.2 \%$ & - & - & - \\
\hline
\end{tabular}

Divide $\mathrm{mg} / \mathrm{kg}$ values by $1 \mathrm{E}+04$ to convert to wt \% dried solids basis 
WSRC-STI-2007-00697, REV. 0

Table 3-5. Composition of the Peroxide Fusion Dissolution of Total Dried Solids from As-Received Tank 51H Sludge (Continued)

\begin{tabular}{|c|c|c|c|c|c|c|c|c|c|c|}
\hline Analyte (Method) & Units & $\begin{array}{c}\text { 1st } \\
\text { Replicate } \\
\end{array}$ & $\begin{array}{c}\text { 2nd } \\
\text { Replicate } \\
\end{array}$ & $\begin{array}{c}\text { 3rd } \\
\text { Replicate } \\
\end{array}$ & $\begin{array}{c}\text { 4th } \\
\text { Replicate } \\
\end{array}$ & Average & \%RSD & Blank & $\begin{array}{c}\text { Average } \\
\text { Analyzed } \\
\text { Glass Std }\end{array}$ & $\begin{array}{c}\text { Glass Std } \\
\text { Composition }\end{array}$ \\
\hline${ }^{99}$ Tc (IM) & $\mathrm{mCi} / \mathrm{kg}$ & $<7.5 \mathrm{E}-02$ & $1.06 \mathrm{E}-01$ & $9.54 \mathrm{E}-02$ & 7.96E-02 & 9.35E-02* & $14 \%$ & - & - & - \\
\hline${ }^{237} \mathrm{~Np}$ (IM) & $\mathrm{mCi} / \mathrm{kg}$ & $7.26 \mathrm{E}-03$ & 7.12E-03 & 7.96E-03 & $7.54 \mathrm{E}-03$ & 7.47E-03 & $5.0 \%$ & - & - & - \\
\hline${ }^{239} \mathrm{Pu}$ (IM) & $\mathrm{mCi} / \mathrm{kg}$ & $4.81 \mathrm{E}+00$ & $5.19 \mathrm{E}+00$ & $5.17 \mathrm{E}+00$ & $4.53 \mathrm{E}+00$ & $4.93 E+00$ & $6.4 \%$ & - & - & - \\
\hline${ }^{240} \mathrm{Pu}(\mathrm{IM})$ & $\mathrm{mCi} / \mathrm{kg}$ & $3.23 \mathrm{E}+00$ & $3.02 \mathrm{E}+00$ & $2.93 \mathrm{E}+00$ & $2.80 \mathrm{E}+00$ & $2.99 \mathrm{E}+00$ & $6.0 \%$ & - & - & - \\
\hline${ }^{242} \mathrm{Pu}$ (IM) & $\mathrm{mCi} / \mathrm{kg}$ & $<1.7 \mathrm{E}-02$ & $<1.7 \mathrm{E}-02$ & $<1.7 \mathrm{E}-02$ & $<1.7 \mathrm{E}-02$ & - & - & - & - & - \\
\hline${ }^{238} \mathrm{Pu}(\mathrm{SA})$ & $\mathrm{mCi} / \mathrm{kg}$ & $2.83 \mathrm{E}+02$ & $3.73 E+02$ & $3.23 E+02$ & $2.87 \mathrm{E}+02$ & $3.17 E+02$ & $13 \%$ & - & - & - \\
\hline${ }^{239 / 240} \mathrm{Pu}(\mathrm{SA})$ & $\mathrm{mCi} / \mathrm{kg}$ & $5.86 \mathrm{E}+00$ & $8.47 \mathrm{E}+00$ & $7.93 \mathrm{E}+00$ & $6.35 \mathrm{E}+00$ & $7.15 \mathrm{E}+00$ & $17 \%$ & - & - & - \\
\hline${ }^{241} \mathrm{Pu}(\mathrm{SA})$ & $\mathrm{mCi} / \mathrm{kg}$ & $9.10 \mathrm{E}+01$ & $1.24 \mathrm{E}+02$ & $1.10 \mathrm{E}+02$ & $9.32 \mathrm{E}+01$ & $1.05 E+02$ & $15 \%$ & - & - & - \\
\hline
\end{tabular}

* - Indicate that not all replicates were used to calculate the Average and the \%RSD. 
WSRC-STI-2007-00697, REV. 0

Table 3-6. Composition of the Filtered Supernate Obtained During the Aluminum Dissolution Demonstration

\begin{tabular}{|c|c|c|c|c|c|c|c|c|}
\hline Analyte (Method) & Units & $\begin{array}{l}0 \text { hour* } \\
\text { Sample }\end{array}$ & $\begin{array}{c}1 \text { hour } \\
\text { Sample }\end{array}$ & $\begin{array}{l}42 \text { hour } \\
\text { Sample }\end{array}$ & $\begin{array}{l}89 \text { hour } \\
\text { Sample }\end{array}$ & $\begin{array}{c}143 \text { hour } \\
\text { Sample }\end{array}$ & $\begin{array}{c}189 \text { hour } \\
\text { Sample }\end{array}$ & $\begin{array}{c}259 \text { hour } \\
\text { Sample }\end{array}$ \\
\hline $\mathrm{Al}$ (IE) & $\mathrm{mg} / \mathrm{L}$ & $2.79 \mathrm{E}+02$ & $3.40 \mathrm{E}+02$ & $2.24 \mathrm{E}+03$ & $3.47 \mathrm{E}+03$ & $4.73 \mathrm{E}+03$ & $5.30 \mathrm{E}+03$ & $6.72 \mathrm{E}+03$ \\
\hline Cr (IE) & $\mathrm{mg} / \mathrm{L}$ & $2.82 \mathrm{E}+01$ & $2.87 \mathrm{E}+01$ & $3.08 \mathrm{E}+01$ & $3.02 \mathrm{E}+01$ & $3.15 \mathrm{E}+01$ & $2.99 \mathrm{E}+01$ & $3.18 \mathrm{E}+01$ \\
\hline Fe (IE) & $\mathrm{mg} / \mathrm{L}$ & $<5.0 \mathrm{E}+00$ & $<2.7 \mathrm{E}+00$ & $1.10 \mathrm{E}+01$ & $3.38 \mathrm{E}+00$ & $3.86 \mathrm{E}+00$ & $3.40 \mathrm{E}+00$ & $<1.2 \mathrm{E}+00$ \\
\hline $\mathrm{Mn}$ (IE) & $\mathrm{mg} / \mathrm{L}$ & $<7.0 \mathrm{E}-01$ & $<1.6 \mathrm{E}+00$ & $8.19 E+00$ & $<1.7 \mathrm{E}+00$ & $<1.7 \mathrm{E}+00$ & $<1.7 \mathrm{E}+00$ & $<7.6 \mathrm{E}-01$ \\
\hline $\mathrm{Na}$ (IE) & $\mathrm{mg} / \mathrm{L}$ & $1.87 \mathrm{E}+04$ & $1.15 \mathrm{E}+05$ & $1.12 \mathrm{E}+05$ & $1.16 \mathrm{E}+05$ & $1.17 \mathrm{E}+05$ & $1.11 \mathrm{E}+05$ & $1.15 \mathrm{E}+05$ \\
\hline U (IE) & $\mathrm{mg} / \mathrm{L}$ & $<6.0 \mathrm{E}+02$ & $<3.3 \mathrm{E}+02$ & $<3.2 \mathrm{E}+02$ & $<3.4 \mathrm{E}+02$ & $<3.5 \mathrm{E}+02$ & $<3.3 \mathrm{E}+02$ & $<1.5 \mathrm{E}+02$ \\
\hline${ }^{233} \mathrm{U}$ (IM) & $\mathrm{mg} / \mathrm{L}$ & $<6.3 \mathrm{E}-02$ & $<2.6 \mathrm{E}-02$ & $<2.5 \mathrm{E}-02$ & $<2.7 \mathrm{E}-02$ & $<2.7 \mathrm{E}-02$ & $<2.6 \mathrm{E}-02$ & $<8.0 \mathrm{E}-03$ \\
\hline${ }^{234} \mathrm{U}$ (IM) & $\mathrm{mg} / \mathrm{L}$ & $<1.7 \mathrm{E}-02$ & $<1.7 \mathrm{E}-02$ & $1.92 \mathrm{E}-02$ & 2.03E-02 & 1.96E-02 & $1.95 \mathrm{E}-02$ & $1.90 \mathrm{E}-02$ \\
\hline${ }^{235} \mathrm{U}$ (IM) & $\mathrm{mg} / \mathrm{L}$ & $<2.3 \mathrm{E}-02$ & 4.93E-02 & $4.44 \mathrm{E}-02$ & 5.60E-02 & $6.13 \mathrm{E}-02$ & $6.02 \mathrm{E}-02$ & $6.22 \mathrm{E}-02$ \\
\hline${ }^{236} \mathrm{U}$ (IM) & $\mathrm{mg} / \mathrm{L}$ & $<1.0 \mathrm{E}-02$ & $<1.7 \mathrm{E}-02$ & $2.00 \mathrm{E}-02$ & $<1.8 \mathrm{E}-02$ & $2.47 \mathrm{E}-02$ & $<1.7 \mathrm{E}-02$ & 1.97E-02 \\
\hline${ }^{238} \mathrm{U}$ (IM) & $\mathrm{mg} / \mathrm{L}$ & $1.15 \mathrm{E}-01$ & $2.51 \mathrm{E}+00$ & $2.86 \mathrm{E}+00$ & $3.45 \mathrm{E}+00$ & $3.47 \mathrm{E}+00$ & $3.61 \mathrm{E}+00$ & $3.84 \mathrm{E}+00$ \\
\hline${ }^{99}$ Tc (IM) & $\mu \mathrm{Ci} / \mathrm{mL}$ & 7.03E-03 & 7.06E-03 & 6.39E-03 & 6.19E-03 & $6.76 \mathrm{E}-03$ & $6.24 \mathrm{E}-03$ & $6.90 \mathrm{E}-03$ \\
\hline${ }^{137} \mathrm{Cs}$ (IM) & $\mu \mathrm{Ci} / \mathrm{mL}$ & $1.71 \mathrm{E}+01$ & $1.71 \mathrm{E}+01$ & $1.69 \mathrm{E}+01$ & $1.73 \mathrm{E}+01$ & $2.00 \mathrm{E}+01$ & $1.75 \mathrm{E}+01$ & $1.84 \mathrm{E}+01$ \\
\hline${ }^{237} \mathrm{~Np}$ (IM) & $\mu \mathrm{Ci} / \mathrm{mL}$ & $<4.0 \mathrm{E}-05$ & 2.99E-05 & $<1.8 \mathrm{E}-05$ & $<1.9 \mathrm{E}-05$ & $<1.9 \mathrm{E}-05$ & $<1.8 \mathrm{E}-05$ & $1.22 \mathrm{E}-05$ \\
\hline${ }^{239} \mathrm{Pu}$ (IM) & $\mu \mathrm{Ci} / \mathrm{mL}$ & $<7.8 \mathrm{E}-04$ & $1.97 \mathrm{E}-02$ & 1.99E-03 & 1.95E-03 & $5.71 \mathrm{E}-03$ & $1.65 \mathrm{E}-03$ & 2.83E-03 \\
\hline${ }^{240} \mathrm{Pu}$ (IM) & $\mu \mathrm{Ci} / \mathrm{mL}$ & $<1.6 \mathrm{E}-02$ & $9.42 \mathrm{E}-03$ & $<3.8 \mathrm{E}-03$ & $<4.0 \mathrm{E}-03$ & $<4.1 \mathrm{E}-03$ & $<3.9 \mathrm{E}-03$ & $<4.5 \mathrm{E}-03$ \\
\hline${ }^{238} \mathrm{Pu}(\mathrm{SA})$ & $\mu \mathrm{Ci} / \mathrm{mL}$ & $9.04 \mathrm{E}-03$ & $6.52 \mathrm{E}-01$ & 4.87E-02 & 7.35E-02 & $7.60 \mathrm{E}-02$ & $6.59 \mathrm{E}-02$ & 7.10E-02 \\
\hline${ }^{239 / 240} \mathrm{Pu}(\mathrm{SA})$ & $\mu \mathrm{Ci} / \mathrm{mL}$ & 4.81E-04 & $3.21 \mathrm{E}-02$ & $2.45 \mathrm{E}-03$ & 3.57E-03 & 8.60E-03 & $3.34 \mathrm{E}-03$ & 4.05E-03 \\
\hline
\end{tabular}

* - The 0 hour concentrations were estimated by adjusting the initial supernate composition from Table 3.3 for the volume of NaOH solution added. 
WSRC-STI-2007-00697, REV. 0

Table 3-6. Composition of the Filtered Supernate Obtained During the Aluminum Dissolution Demonstration (Continued)

\begin{tabular}{|c|c|c|c|c|c|c|c|}
\hline Analyte (Method) & Units & $\begin{array}{c}308 \text { hour } \\
\text { Sample }\end{array}$ & $\begin{array}{c}358 \text { hour } \\
\text { Sample }\end{array}$ & $\begin{array}{c}400 \text { hour } \\
\text { Sample }\end{array}$ & $\begin{array}{c}453 \text { hour } \\
\text { Sample }\end{array}$ & $\begin{array}{c}495 \text { hour } \\
\text { Sample }\end{array}$ & $\begin{array}{c}\text { Final } \\
\text { Decant }\end{array}$ \\
\hline $\mathrm{Al}$ (IE) & $\mathrm{mg} / \mathrm{L}$ & $7.34 \mathrm{E}+03$ & $7.94 \mathrm{E}+03$ & $8.43 \mathrm{E}+03$ & $8.83 \mathrm{E}+03$ & $9.28 \mathrm{E}+03$ & $9.58 \mathrm{E}+03$ \\
\hline Cr (IE) & $\mathrm{mg} / \mathrm{L}$ & $3.20 \mathrm{E}+01$ & $3.24 \mathrm{E}+01$ & $3.30 \mathrm{E}+01$ & $3.31 \mathrm{E}+01$ & $3.35 E+01$ & $3.51 \mathrm{E}+01$ \\
\hline Fe (IE) & $\mathrm{mg} / \mathrm{L}$ & $1.79 \mathrm{E}+00$ & $1.27 \mathrm{E}+00$ & $1.28 \mathrm{E}+00$ & $2.31 \mathrm{E}+00$ & $1.49 \mathrm{E}+00$ & $4.81 \mathrm{E}+00$ \\
\hline Mn (IE) & $\mathrm{mg} / \mathrm{L}$ & 7.86E-01 & $<7.8 \mathrm{E}-01$ & $<7.9 \mathrm{E}-01$ & $<7.9 \mathrm{E}-01$ & $<8.9 \mathrm{E}-01$ & $<5.9 \mathrm{E}-01$ \\
\hline $\mathrm{Na}$ (IE) & $\mathrm{mg} / \mathrm{L}$ & $1.15 \mathrm{E}+05$ & $1.16 \mathrm{E}+05$ & $1.17 \mathrm{E}+05$ & $1.15 \mathrm{E}+05$ & $1.17 \mathrm{E}+05$ & $1.21 \mathrm{E}+05$ \\
\hline U (IE) & $\mathrm{mg} / \mathrm{L}$ & $<1.6 \mathrm{E}+02$ & $<1.6 \mathrm{E}+02$ & $<1.6 \mathrm{E}+02$ & $<1.6 \mathrm{E}+02$ & $<1.8 \mathrm{E}+02$ & $<5.1 \mathrm{E}+02$ \\
\hline${ }^{233} \mathrm{U}$ (IM) & $\mathrm{mg} / \mathrm{L}$ & $<8.2 \mathrm{E}-03$ & $<8.1 \mathrm{E}-03$ & $<8.2 \mathrm{E}-03$ & $<8.2 \mathrm{E}-03$ & $<9.3 \mathrm{E}-03$ & $<1.5 \mathrm{E}-01$ \\
\hline${ }^{234} \mathrm{U}$ (IM) & $\mathrm{mg} / \mathrm{L}$ & $2.05 \mathrm{E}-02$ & $1.43 \mathrm{E}-02$ & $1.70 \mathrm{E}-02$ & $1.44 \mathrm{E}-02$ & $1.86 \mathrm{E}-02$ & $<6.7 \mathrm{E}-02$ \\
\hline${ }^{235} \mathrm{U}$ (IM) & $\mathrm{mg} / \mathrm{L}$ & 5.65E-02 & $5.12 \mathrm{E}-02$ & $4.93 \mathrm{E}-02$ & 3.84E-02 & $4.83 \mathrm{E}-02$ & $<1.2 \mathrm{E}-01$ \\
\hline${ }^{236} \mathrm{U}$ (IM) & $\mathrm{mg} / \mathrm{L}$ & $1.86 \mathrm{E}-02$ & $1.59 \mathrm{E}-02$ & $<8.2 \mathrm{E}-03$ & $1.71 \mathrm{E}-02$ & $1.80 \mathrm{E}-02$ & $<3.4 \mathrm{E}-02$ \\
\hline${ }^{238} \mathrm{U}$ (IM) & $\mathrm{mg} / \mathrm{L}$ & $3.72 E+00$ & $3.58 \mathrm{E}+00$ & $3.67 \mathrm{E}+00$ & $3.52 \mathrm{E}+00$ & $3.46 \mathrm{E}+00$ & $3.33 \mathrm{E}+00$ \\
\hline${ }^{99}$ Tc (IM) & $\mu \mathrm{Ci} / \mathrm{mL}$ & $6.77 \mathrm{E}-03$ & $6.79 \mathrm{E}-03$ & $6.64 \mathrm{E}-03$ & 6.73E-03 & $6.36 \mathrm{E}-03$ & 8.16E-03 \\
\hline${ }^{137} \mathrm{Cs}$ (IM) & $\mu \mathrm{Ci} / \mathrm{mL}$ & $1.93 \mathrm{E}+01$ & $1.81 \mathrm{E}+01$ & $1.90 \mathrm{E}+01$ & $1.79 \mathrm{E}+01$ & $1.88 \mathrm{E}+01$ & $1.80 \mathrm{E}+01$ \\
\hline${ }^{237} \mathrm{~Np}$ (IM) & $\mu \mathrm{Ci} / \mathrm{mL}$ & $<8.7 \mathrm{E}-06$ & $1.34 \mathrm{E}-05$ & $1.24 \mathrm{E}-05$ & $1.21 \mathrm{E}-05$ & $1.29 \mathrm{E}-05$ & $<4.8 \mathrm{E}-05$ \\
\hline${ }^{239} \mathrm{Pu}$ (IM) & $\mu \mathrm{Ci} / \mathrm{mL}$ & $<5.0 \mathrm{E}-04$ & $2.74 \mathrm{E}-03$ & 2.38E-03 & 2.68E-03 & 2.37E-03 & $3.00 \mathrm{E}-03$ \\
\hline${ }^{240} \mathrm{Pu}$ (IM) & $\mu \mathrm{Ci} / \mathrm{mL}$ & $<4.6 \mathrm{E}-03$ & $<4.6 \mathrm{E}-03$ & $<4.6 \mathrm{E}-03$ & $<4.7 \mathrm{E}-03$ & $<5.2 \mathrm{E}-03$ & $<2.7 \mathrm{E}-02$ \\
\hline${ }^{238} \mathrm{Pu}(\mathrm{SA})$ & $\mu \mathrm{Ci} / \mathrm{mL}$ & 8.30E-02 & 7.71E-02 & $6.86 \mathrm{E}-02$ & 7.02E-02 & 6.83E-02 & 7.36E-02 \\
\hline${ }^{239 / 240} \mathrm{Pu}(\mathrm{SA})$ & $\mu \mathrm{Ci} / \mathrm{mL}$ & $4.02 \mathrm{E}-03$ & 3.93E-03 & $3.66 \mathrm{E}-03$ & 3.86E-03 & 3.67E-03 & 3.59E-03 \\
\hline
\end{tabular}

* - The 0 hour concentrations were estimated by adjusting the initial supernate composition from Table 3.3 for the volume of NaOH solution added. 
WSRC-STI-2007-00697, REV. 0

Table 3-7. Weight Percent Solids and Density of the Post Aluminum Dissolution Tank 51H Sludge and Decanted Supernate

\begin{tabular}{|c|c|c|c|c|c|c|c|}
\hline Analyte (Method) & Units & $\begin{array}{c}\text { 1st } \\
\text { Replicate }\end{array}$ & $\begin{array}{c}\text { 2nd } \\
\text { Replicate }\end{array}$ & $\begin{array}{c}\text { 3rd } \\
\text { Replicate }\end{array}$ & $\begin{array}{c}\text { 4th } \\
\text { Replicate }\end{array}$ & Average & \%RSD \\
\hline Weight Percent Total Solids & $\mathrm{Wt} \%$ & 28.0 & 28.1 & 27.9 & 28.0 & 28.0 & $0.2 \%$ \\
\hline Weight Percent Dissolve Solids & $\mathrm{Wt} \%$ & 23.0 & 23.5 & 22.6 & 23.0 & 23.0 & $1.7 \%$ \\
\hline Weight Percent Soluble Solids & $\mathrm{Wt} \%$ & - & - & - & - & $21.5^{*}$ & - \\
\hline Weight Percent Insoluble & $\mathrm{Wt} \%$ & - & - & - & - & $6.44 *$ & - \\
\hline Density of Slurry & $\mathrm{g} / \mathrm{mL}$ & 1.26 & 1.25 & 1.28 & 1.27 & 1.27 & $1.2 \%$ \\
\hline Density of Supernate & $\mathrm{g} / \mathrm{mL}$ & 1.20 & 1.21 & 1.21 & 1.22 & 1.21 & $0.6 \%$ \\
\hline
\end{tabular}

* Values for the weight percent soluble solids and weight percent insoluble solids were calculated from the measured weight percent total solids and weight percent dissolved solids (see Section 2.4) 
WSRC-STI-2007-00697, REV. 0

Table 3-8. Composition of the Post Aluminum Dissolution Filtered Supernate

\begin{tabular}{|c|c|c|c|c|c|c|c|c|}
\hline Analyte (Method) & Units & $\begin{array}{c}\text { 1st } \\
\text { Replicate }\end{array}$ & $\begin{array}{c}\text { 2nd } \\
\text { Replicate }\end{array}$ & $\begin{array}{c}\text { 3rd } \\
\text { Replicate }\end{array}$ & $\begin{array}{c}\text { 4th } \\
\text { Replicate }\end{array}$ & Average & \%RSD & Blank \\
\hline $\mathrm{NO}_{3}^{-}$(IC) & $\mathrm{M}$ & $2.15 \mathrm{E}-01$ & $2.13 \mathrm{E}-01$ & 2.32E-01 & 2.13E-01 & $2.18 \mathrm{E}-01$ & $4.3 \%$ & $<1.9 \mathrm{E}-04$ \\
\hline $\mathrm{NO}_{2}^{-}$(IC) & $\mathrm{M}$ & $4.21 \mathrm{E}-01$ & $4.00 \mathrm{E}-01$ & 4.42E-01 & 3.97E-01 & 4.15E-01 & $5.0 \%$ & $<2.6 \mathrm{E}-04$ \\
\hline $\mathrm{PO}_{4}^{3-}$ (IC) & M & $<1.2 \mathrm{E}-02$ & $<1.3 \mathrm{E}-02$ & $<1.3 \mathrm{E}-02$ & $<1.3 \mathrm{E}-02$ & - & - & $<3.2 \mathrm{E}-04$ \\
\hline $\mathrm{SO}_{4}^{2-}(\mathrm{IC})$ & $M$ & $2.57 \mathrm{E}-02$ & $2.08 \mathrm{E}-02$ & $1.90 \mathrm{E}-02$ & $2.05 \mathrm{E}-02$ & 2.15E-02 & $13 \%$ & $<1.2 \mathrm{E}-04$ \\
\hline $\mathrm{C}_{2} \mathrm{O}_{4}{ }^{2-}$ (IC) & $M$ & $<1.4 \mathrm{E}-02$ & $<1.4 \mathrm{E}-02$ & $<1.4 \mathrm{E}-02$ & $<1.4 \mathrm{E}-02$ & - & - & $<1.4 \mathrm{E}-04$ \\
\hline $\mathrm{Cl}^{-}$(IC) & M & $<3.4 \mathrm{E}-02$ & $<3.4 \mathrm{E}-02$ & $<3.4 \mathrm{E}-02$ & $<3.4 \mathrm{E}-02$ & - & - & $<3.4 \mathrm{E}-04$ \\
\hline $\mathrm{F}^{-}$(IC) & M & $<6.4 \mathrm{E}-02$ & $<6.4 \mathrm{E}-02$ & $<6.4 \mathrm{E}-02$ & $<6.4 \mathrm{E}-02$ & - & - & $<6.3 \mathrm{E}-04$ \\
\hline $\mathrm{CHO}_{2}^{-}(\mathrm{IC})$ & M & $<2.7 \mathrm{E}-02$ & $<2.7 \mathrm{E}-02$ & $<2.7 \mathrm{E}-02$ & $<2.7 \mathrm{E}-02$ & - & - & $<2.7 \mathrm{E}-04$ \\
\hline $\mathrm{OH}_{\text {free }}^{-}(\mathrm{T})$ & M & $3.79 \mathrm{E}+00$ & $3.85 E+00$ & $3.88 \mathrm{E}+00$ & $3.76 \mathrm{E}+00$ & $3.82 E+00$ & $1.4 \%$ & $<2.4 \mathrm{E}-04$ \\
\hline Ag (IE) & $\mathrm{mg} / \mathrm{L}$ & $<7.7 \mathrm{E}+00$ & $<7.8 \mathrm{E}+00$ & $<7.1 \mathrm{E}+00$ & $<7.0 \mathrm{E}+00$ & - & - & $<7.4 \mathrm{E}+00$ \\
\hline Al (IE) & $\mathrm{mg} / \mathrm{L}$ & $9.54 \mathrm{E}+03$ & $9.52 E+03$ & $9.57 \mathrm{E}+03$ & $9.70 \mathrm{E}+03$ & $9.58 E+03$ & $0.8 \%$ & $<2.6 \mathrm{E}+01$ \\
\hline B (IE) & $\mathrm{mg} / \mathrm{L}$ & $<6.6 \mathrm{E}+00$ & $<6.7 \mathrm{E}+00$ & $<6.1 \mathrm{E}+00$ & $<6.0 \mathrm{E}+00$ & - & - & $<6.4 \mathrm{E}+00$ \\
\hline Ba (IE) & $\mathrm{mg} / \mathrm{L}$ & $<1.7 \mathrm{E}+00$ & $<1.7 \mathrm{E}+00$ & $<1.5 \mathrm{E}+00$ & $<1.5 \mathrm{E}+00$ & - & - & $<1.6 \mathrm{E}+00$ \\
\hline Be (IE) & $\mathrm{mg} / \mathrm{L}$ & $<2.9 \mathrm{E}-01$ & $<3.0 \mathrm{E}-01$ & $<2.7 \mathrm{E}-01$ & $<2.7 \mathrm{E}-01$ & - & - & $<2.8 \mathrm{E}-01$ \\
\hline Ca (IE) & $\mathrm{mg} / \mathrm{L}$ & $<2.6 \mathrm{E}+01$ & $<2.6 \mathrm{E}+01$ & $<2.4 \mathrm{E}+01$ & $<2.3 \mathrm{E}+01$ & - & - & $<2.5 \mathrm{E}+01$ \\
\hline Cd (IE) & $\mathrm{mg} / \mathrm{L}$ & $3.08 \mathrm{E}+00$ & $3.06 \mathrm{E}+00$ & $3.02 E+00$ & $2.92 \mathrm{E}+00$ & $3.02 E+00$ & $2.4 \%$ & $<2.1 \mathrm{E}+00$ \\
\hline Ce (IE) & $\mathrm{mg} / \mathrm{L}$ & $<1.5 \mathrm{E}+02$ & $<1.5 \mathrm{E}+02$ & $<1.4 \mathrm{E}+02$ & $<1.4 \mathrm{E}+02$ & - & - & $<1.4 \mathrm{E}+02$ \\
\hline Cr (IE) & $\mathrm{mg} / \mathrm{L}$ & $3.50 \mathrm{E}+01$ & $3.49 \mathrm{E}+01$ & $3.51 \mathrm{E}+01$ & $3.55 \mathrm{E}+01$ & $3.51 \mathrm{E}+01$ & $0.7 \%$ & $<2.6 \mathrm{E}+00$ \\
\hline $\mathrm{Cu}$ (IE) & $\mathrm{mg} / \mathrm{L}$ & $<4.4 \mathrm{E}+00$ & $<4.4 \mathrm{E}+00$ & $<4.0 \mathrm{E}+00$ & $<3.9 \mathrm{E}+00$ & - & - & $<4.2 \mathrm{E}+00$ \\
\hline Fe (IE) & $\mathrm{mg} / \mathrm{L}$ & $4.74 \mathrm{E}+00$ & $5.07 \mathrm{E}+00$ & $4.52 \mathrm{E}+00$ & $4.90 \mathrm{E}+00$ & $4.81 E+00$ & $5.0 \%$ & $<4.0 \mathrm{E}+00$ \\
\hline
\end{tabular}

* - Indicate that not all replicates were used to calculate the Average and the \%RSD. 
WSRC-STI-2007-00697, REV. 0

Table 3-8. Composition of the Post Aluminum Dissolution Filtered Supernate (Continued)

\begin{tabular}{|c|c|c|c|c|c|c|c|c|}
\hline Analyte (Method) & Units & $\begin{array}{c}\text { 1st } \\
\text { Replicate }\end{array}$ & $\begin{array}{c}\text { 2nd } \\
\text { Replicate }\end{array}$ & $\begin{array}{c}\text { 3rd } \\
\text { Replicate }\end{array}$ & $\begin{array}{c}\text { 4th } \\
\text { Replicate }\end{array}$ & Average & \%RSD & Blank \\
\hline Gd (IE) & $\mathrm{mg} / \mathrm{L}$ & $<9.2 \mathrm{E}+00$ & $<9.3 \mathrm{E}+00$ & $<8.4 \mathrm{E}+00$ & $<8.3 \mathrm{E}+00$ & - & - & $<8.8 \mathrm{E}+00$ \\
\hline K (IE) & $\mathrm{mg} / \mathrm{L}$ & $<1.3 \mathrm{E}+02$ & $<1.3 \mathrm{E}+02$ & $<1.2 \mathrm{E}+02$ & $<1.1 \mathrm{E}+02$ & - & - & $<1.2 \mathrm{E}+02$ \\
\hline La (IE) & $\mathrm{mg} / \mathrm{L}$ & $<1.1 \mathrm{E}+01$ & $<1.1 \mathrm{E}+01$ & $<1.0 \mathrm{E}+01$ & $<1.0 \mathrm{E}+01$ & - & - & $<1.1 \mathrm{E}+01$ \\
\hline Li (IE) & $\mathrm{mg} / \mathrm{L}$ & $<7.8 \mathrm{E}+00$ & $<7.9 \mathrm{E}+00$ & $<7.1 \mathrm{E}+00$ & $<7.0 \mathrm{E}+00$ & - & - & $<7.5 \mathrm{E}+00$ \\
\hline $\mathrm{Mg}$ (IE) & $\mathrm{mg} / \mathrm{L}$ & $<9.1 \mathrm{E}-01$ & $<9.2 \mathrm{E}-01$ & $<8.3 \mathrm{E}-01$ & $<8.2 \mathrm{E}-01$ & - & - & $<8.7 \mathrm{E}-01$ \\
\hline $\mathrm{Mn}$ (IE) & $\mathrm{mg} / \mathrm{L}$ & $<5.8 \mathrm{E}-01$ & $<5.9 \mathrm{E}-01$ & $<5.4 \mathrm{E}-01$ & $<5.3 \mathrm{E}-01$ & - & - & $<5.6 \mathrm{E}-01$ \\
\hline Mo (IE) & $\mathrm{mg} / \mathrm{L}$ & $<8.4 \mathrm{E}+00$ & $<8.5 \mathrm{E}+00$ & $<7.7 \mathrm{E}+00$ & $<7.6 \mathrm{E}+00$ & - & - & $<8.0 \mathrm{E}+00$ \\
\hline $\mathrm{Na}$ (IE) & $\mathrm{mg} / \mathrm{L}$ & $1.20 \mathrm{E}+05$ & $1.20 \mathrm{E}+05$ & $1.20 \mathrm{E}+05$ & $1.22 \mathrm{E}+05$ & $1.21 \mathrm{E}+05$ & $1.0 \%$ & $<7.1 \mathrm{E}+01$ \\
\hline $\mathrm{Ni}$ (IE) & $\mathrm{mg} / \mathrm{L}$ & $<1.4 \mathrm{E}+01$ & $<1.4 \mathrm{E}+01$ & $<1.2 \mathrm{E}+01$ & $<1.2 \mathrm{E}+01$ & - & - & $<1.3 \mathrm{E}+01$ \\
\hline $\mathrm{P}$ (IE) & $\mathrm{mg} / \mathrm{L}$ & $3.74 \mathrm{E}+01$ & $4.14 \mathrm{E}+01$ & $<3.6 \mathrm{E}+01$ & $3.97 \mathrm{E}+01$ & $3.95 \mathrm{E}+01 *$ & $5.1 \%$ & $<3.7 \mathrm{E}+01$ \\
\hline $\mathrm{Pb}$ (IE) & $\mathrm{mg} / \mathrm{L}$ & $<2.2 \mathrm{E}+01$ & $<2.2 \mathrm{E}+01$ & $<2.0 \mathrm{E}+01$ & $<2.0 \mathrm{E}+01$ & - & - & $<2.1 \mathrm{E}+01$ \\
\hline S (IE) & $\mathrm{mg} / \mathrm{L}$ & $6.45 \mathrm{E}+02$ & $6.30 \mathrm{E}+02$ & $6.41 \mathrm{E}+02$ & $6.35 \mathrm{E}+02$ & $6.38 \mathrm{E}+02$ & $1.1 \%$ & $<3.0 \mathrm{E}+02$ \\
\hline $\mathrm{Sb}$ (IE) & $\mathrm{mg} / \mathrm{L}$ & $<4.2 \mathrm{E}+01$ & $<4.2 \mathrm{E}+01$ & $<3.8 \mathrm{E}+01$ & $<3.8 \mathrm{E}+01$ & - & - & $<4.0 \mathrm{E}+01$ \\
\hline Si (IE) & $\mathrm{mg} / \mathrm{L}$ & $<7.7 \mathrm{E}+01$ & $<7.8 \mathrm{E}+01$ & $<7.1 \mathrm{E}+01$ & $<7.0 \mathrm{E}+01$ & - & - & $<7.4 \mathrm{E}+01$ \\
\hline Sn (IE) & $\mathrm{mg} / \mathrm{L}$ & $<1.2 \mathrm{E}+02$ & $<1.3 \mathrm{E}+02$ & $<1.1 \mathrm{E}+02$ & $<1.1 \mathrm{E}+02$ & - & - & $<1.2 \mathrm{E}+02$ \\
\hline Sr (IE) & $\mathrm{mg} / \mathrm{L}$ & $<1.1 \mathrm{E}+01$ & $<1.1 \mathrm{E}+01$ & $<1.0 \mathrm{E}+01$ & $<1.0 \mathrm{E}+01$ & - & - & $<1.1 \mathrm{E}+01$ \\
\hline Ti (IE) & $\mathrm{mg} / \mathrm{L}$ & $<2.5 \mathrm{E}+00$ & $<2.5 \mathrm{E}+00$ & $<2.3 \mathrm{E}+00$ & $<2.2 \mathrm{E}+00$ & - & - & $<2.4 \mathrm{E}+00$ \\
\hline U (IE) & $\mathrm{mg} / \mathrm{L}$ & $<5.0 \mathrm{E}+02$ & $<5.1 \mathrm{E}+02$ & $<4.6 \mathrm{E}+02$ & $<4.6 \mathrm{E}+02$ & - & - & $<4.8 \mathrm{E}+02$ \\
\hline V (IE) & $\mathrm{mg} / \mathrm{L}$ & $<4.8 \mathrm{E}+00$ & $<4.9 \mathrm{E}+00$ & $<4.4 \mathrm{E}+00$ & $<4.4 \mathrm{E}+00$ & - & - & $<4.6 \mathrm{E}+00$ \\
\hline $\mathrm{Zn}$ (IE) & $\mathrm{mg} / \mathrm{L}$ & $<2.7 \mathrm{E}+01$ & $<2.7 \mathrm{E}+01$ & $<2.5 \mathrm{E}+01$ & $<2.5 \mathrm{E}+01$ & - & - & $<2.6 \mathrm{E}+01$ \\
\hline Zr (IE) & $\mathrm{mg} / \mathrm{L}$ & $<3.0 \mathrm{E}+00$ & $<3.0 \mathrm{E}+00$ & $<2.7 \mathrm{E}+00$ & $<2.7 \mathrm{E}+00$ & - & - & $<2.9 \mathrm{E}+00$ \\
\hline
\end{tabular}

* - Indicate that not all replicates were used to calculate the Average and the \%RSD. 
WSRC-STI-2007-00697, REV. 0

Table 3-8. Composition of the Post Aluminum Dissolution Filtered Supernate (Continued)

\begin{tabular}{|c|c|c|c|c|c|c|c|c|}
\hline Analyte (Method) & Units & $\begin{array}{c}\text { 1st } \\
\text { Replicate }\end{array}$ & $\begin{array}{c}\text { 2nd } \\
\text { Replicate }\end{array}$ & $\begin{array}{c}\text { 3rd } \\
\text { Replicate }\end{array}$ & $\begin{array}{c}\text { 4th } \\
\text { Replicate }\end{array}$ & Average & \%RSD & Blank \\
\hline $\mathrm{Hg}(\mathrm{CV})$ & $\mathrm{mg} / \mathrm{L}$ & $2.00 \mathrm{E}+02$ & $2.00 \mathrm{E}+02$ & $2.10 \mathrm{E}+02$ & $1.99 \mathrm{E}+02$ & $2.02 E+02$ & $2.5 \%$ & $<2.5 \mathrm{E}-03$ \\
\hline $\mathrm{Na}$ (AA) & $\mathrm{mg} / \mathrm{L}$ & $1.11 \mathrm{E}+05$ & $1.09 \mathrm{E}+05$ & $1.13 E+05$ & $1.14 \mathrm{E}+05$ & 1.12E+05 & $2.1 \%$ & 4.04E-02 \\
\hline${ }^{233} \mathrm{U}$ (IM) & $\mathrm{mg} / \mathrm{L}$ & $<1.5 \mathrm{E}-01$ & $<1.5 \mathrm{E}-01$ & $<1.4 \mathrm{E}-01$ & $<1.3 \mathrm{E}-01$ & - & - & $<1.4 \mathrm{E}-02$ \\
\hline${ }^{234} \mathrm{U}$ (IM) & $\mathrm{mg} / \mathrm{L}$ & $<6.6 \mathrm{E}-02$ & $<6.7 \mathrm{E}-02$ & $<6.1 \mathrm{E}-02$ & $<6.0 \mathrm{E}-02$ & - & - & $<6.3 \mathrm{E}-03$ \\
\hline${ }^{235} \mathrm{U}$ (IM) & $\mathrm{mg} / \mathrm{L}$ & $<1.2 \mathrm{E}-01$ & $<1.2 \mathrm{E}-01$ & $<1.1 \mathrm{E}-01$ & $<1.0 \mathrm{E}-01$ & - & - & $<1.1 \mathrm{E}-02$ \\
\hline${ }^{236} \mathrm{U}$ (IM) & $\mathrm{mg} / \mathrm{L}$ & $<3.3 \mathrm{E}-02$ & $<3.4 \mathrm{E}-02$ & $<3.0 \mathrm{E}-02$ & $<3.0 \mathrm{E}-02$ & - & - & $<3.2 \mathrm{E}-03$ \\
\hline${ }^{238} \mathrm{U}$ (IM) & $\mathrm{mg} / \mathrm{L}$ & $3.43 \mathrm{E}+00$ & $3.15 E+00$ & $3.35 \mathrm{E}+00$ & $3.40 \mathrm{E}+00$ & $3.33 E+00$ & $3.8 \%$ & $<7.9 \mathrm{E}-03$ \\
\hline${ }^{99}$ Tc (IM) & $\mu \mathrm{Ci} / \mathrm{mL}$ & 7.75E-03 & 8.26E-03 & 8.53E-03 & 8.12E-03 & 8.16E-03 & $4.0 \%$ & $<1.9 \mathrm{E}-04$ \\
\hline${ }^{137}$ Cs (IM) & $\mu \mathrm{Ci} / \mathrm{mL}$ & $1.62 \mathrm{E}+01$ & $2.04 \mathrm{E}+01$ & $1.79 \mathrm{E}+01$ & $1.74 \mathrm{E}+01$ & $1.80 \mathrm{E}+01$ & $10 \%$ & $<6.9 \mathrm{E}-01$ \\
\hline${ }^{237} \mathrm{~Np}$ (IM) & $\mu \mathrm{Ci} / \mathrm{mL}$ & $<4.7 \mathrm{E}-05$ & $<4.8 \mathrm{E}-05$ & $<4.3 \mathrm{E}-05$ & $<4.2 \mathrm{E}-05$ & - & - & $<4.5 \mathrm{E}-06$ \\
\hline${ }^{239} \mathrm{Pu}$ (IM) & $\mu \mathrm{Ci} / \mathrm{mL}$ & 3.27E-03 & $<2.1 \mathrm{E}-03$ & 3.19E-03 & $2.52 \mathrm{E}-03$ & $3.00 \mathrm{E}-03^{*}$ & $14 \%$ & $<1.9 \mathrm{E}-04$ \\
\hline${ }^{240} \mathrm{Pu}$ (IM) & $\mu \mathrm{Ci} / \mathrm{mL}$ & $<2.6 \mathrm{E}-02$ & $<2.7 \mathrm{E}-02$ & $<2.4 \mathrm{E}-02$ & $<2.4 \mathrm{E}-02$ & - & - & $<2.5 \mathrm{E}-03$ \\
\hline${ }^{238} \mathrm{Pu}(\mathrm{SA})$ & $\mu \mathrm{Ci} / \mathrm{mL}$ & 7.36E-02 & 7.09E-02 & $7.20 \mathrm{E}-02$ & $7.80 \mathrm{E}-02$ & 7.36E-02 & $4.2 \%$ & $<4.3 \mathrm{E}-07$ \\
\hline${ }^{239 / 240} \mathrm{Pu}(\mathrm{SA})$ & $\mu \mathrm{Ci} / \mathrm{mL}$ & $3.52 \mathrm{E}-03$ & $3.70 \mathrm{E}-03$ & $3.41 \mathrm{E}-03$ & $3.71 \mathrm{E}-03$ & 3.59E-03 & $4.1 \%$ & $2.62 \mathrm{E}-07$ \\
\hline${ }^{241} \mathrm{Pu}$ (SA) & $\mu \mathrm{Ci} / \mathrm{mL}$ & 2.53E-02 & 2.53E-02 & $2.51 \mathrm{E}-02$ & $3.00 \mathrm{E}-02$ & 2.64E-02 & $9.0 \%$ & $<1.1 \mathrm{E}-05$ \\
\hline${ }^{90} \mathrm{Sr}$ (SL) & $\mu \mathrm{Ci} / \mathrm{mL}$ & $9.06 \mathrm{E}-01$ & $1.01 \mathrm{E}+00$ & $1.02 \mathrm{E}+00$ & $9.27 \mathrm{E}-01$ & 9.67E-01 & $6.1 \%$ & $4.02 \mathrm{E}-05$ \\
\hline${ }^{232} \mathrm{U}(\mathrm{SL})$ & $\mu \mathrm{Ci} / \mathrm{mL}$ & $<6.7 \mathrm{E}-06$ & $<2.7 \mathrm{E}-05$ & $<4.0 \mathrm{E}-06$ & $<5.2 \mathrm{E}-06$ & - & - & $<1.9 \mathrm{E}-06$ \\
\hline Total Alpha (SL) & $\mu \mathrm{Ci} / \mathrm{mL}$ & $<1.6 \mathrm{E}-01$ & $<1.4 \mathrm{E}-01$ & $<1.9 \mathrm{E}-01$ & $<1.2 \mathrm{E}-01$ & - & - & - \\
\hline Total Beta (SL) & $\mu \mathrm{Ci} / \mathrm{mL}$ & $2.18 \mathrm{E}+01$ & $2.07 \mathrm{E}+01$ & $2.10 \mathrm{E}+01$ & $2.23 \mathrm{E}+01$ & $2.14 \mathrm{E}+01$ & $3.5 \%$ & - \\
\hline
\end{tabular}

* - Indicate that not all replicates were used to calculate the Average and the \%RSD. 
WSRC-STI-2007-00697, REV. 0

Table 3-8. Composition of the Post Aluminum Dissolution Filtered Supernate (Continued)

\begin{tabular}{|c|c|c|c|c|c|c|c|c|}
\hline Analyte (Method) & Units & $\begin{array}{c}\text { 1st } \\
\text { Replicate }\end{array}$ & $\begin{array}{c}\text { 2nd } \\
\text { Replicate }\end{array}$ & $\begin{array}{c}\text { 3rd } \\
\text { Replicate }\end{array}$ & $\begin{array}{c}\text { 4th } \\
\text { Replicate }\end{array}$ & Average & \%RSD & Blank \\
\hline${ }^{137} \mathrm{Cs}$ (SG) & $\mu \mathrm{Ci} / \mathrm{mL}$ & $1.84 \mathrm{E}+01$ & $1.91 \mathrm{E}+01$ & $1.86 \mathrm{E}+01$ & $1.92 \mathrm{E}+01$ & $1.88 \mathrm{E}+01$ & $2.0 \%$ & $<1.1 \mathrm{E}-04$ \\
\hline${ }^{134}$ Cs (SG) & $\mu \mathrm{Ci} / \mathrm{mL}$ & $<9.7 \mathrm{E}-03$ & $<1.1 \mathrm{E}-02$ & $<1.0 \mathrm{E}-02$ & $<9.4 \mathrm{E}-03$ & - & - & $<1.0 \mathrm{E}-04$ \\
\hline${ }^{40} \mathrm{~K}$ (SG) & $\mu \mathrm{Ci} / \mathrm{mL}$ & - & $1.98 \mathrm{E}-03$ & $1.51 \mathrm{E}-03$ & - & $1.75 \mathrm{E}-03^{*}$ & $19 \%$ & $<1.6 \mathrm{E}-06$ \\
\hline${ }^{60} \mathrm{Co}$ (SG) & $\mu \mathrm{Ci} / \mathrm{mL}$ & $<5.1 \mathrm{E}-05$ & $<5.0 \mathrm{E}-05$ & $<4.4 \mathrm{E}-05$ & $<4.7 \mathrm{E}-05$ & - & - & $<4.1 \mathrm{E}-07$ \\
\hline${ }^{106} \mathrm{Ru}$ (SG) & $\mu \mathrm{Ci} / \mathrm{mL}$ & $<3.6 \mathrm{E}-04$ & $<3.5 \mathrm{E}-04$ & $<3.4 \mathrm{E}-04$ & $<3.2 \mathrm{E}-04$ & - & - & $<2.7 \mathrm{E}-06$ \\
\hline${ }^{125} \mathrm{Sb}(\mathrm{SG})$ & $\mu \mathrm{Ci} / \mathrm{mL}$ & $<1.8 \mathrm{E}-04$ & $<1.7 \mathrm{E}-04$ & $<1.6 \mathrm{E}-04$ & $<1.6 \mathrm{E}-04$ & - & - & $<1.1 \mathrm{E}-06$ \\
\hline${ }^{126} \mathrm{Sb}(\mathrm{SG})$ & $\mu \mathrm{Ci} / \mathrm{mL}$ & 6.49E-05 & 8.62E-05 & 7.04E-05 & 7.69E-05 & $7.46 \mathrm{E}-05$ & $12 \%$ & $<3.8 \mathrm{E}-07$ \\
\hline${ }^{126} \mathrm{Sn}$ (SG) & $\mu \mathrm{Ci} / \mathrm{mL}$ & 6.49E-05 & 8.62E-05 & $7.04 \mathrm{E}-05$ & 7.69E-05 & $7.46 \mathrm{E}-05$ & $12 \%$ & $<3.8 \mathrm{E}-07$ \\
\hline${ }^{144} \mathrm{Ce}$ (SG) & $\mu \mathrm{Ci} / \mathrm{mL}$ & $<6.2 \mathrm{E}-04$ & $<6.2 \mathrm{E}-04$ & $<5.8 \mathrm{E}-04$ & $<5.9 \mathrm{E}-04$ & - & - & $<1.7 \mathrm{E}-06$ \\
\hline${ }^{154} \mathrm{Eu}(\mathrm{SG})$ & $\mu \mathrm{Ci} / \mathrm{mL}$ & $1.72 \mathrm{E}-04$ & $<1.9 \mathrm{E}-04$ & $2.05 \mathrm{E}-04$ & $<1.6 \mathrm{E}-04$ & $1.88 \mathrm{E}-04 *$ & $12 \%$ & $<4.7 \mathrm{E}-07$ \\
\hline${ }^{155} \mathrm{Eu}(\mathrm{SG})$ & $\mu \mathrm{Ci} / \mathrm{mL}$ & $<3.2 \mathrm{E}-04$ & $<3.2 \mathrm{E}-04$ & $<3.0 \mathrm{E}-04$ & $<3.0 \mathrm{E}-04$ & - & - & $<7.9 \mathrm{E}-07$ \\
\hline${ }^{241} \mathrm{Am}$ (SG) & $\mu \mathrm{Ci} / \mathrm{mL}$ & $<6.6 \mathrm{E}-04$ & $<6.7 \mathrm{E}-04$ & $<6.3 \mathrm{E}-04$ & $<6.3 \mathrm{E}-04$ & - & - & $<1.6 \mathrm{E}-06$ \\
\hline
\end{tabular}

* - Indicate that not all replicates were used to calculate the Average and the \%RSD. 
WSRC-STI-2007-00697, REV. 0

Table 3-9. Composition of the Aqua Regia Dissolution of Total Dried Solids from the Post Aluminum Dissolution Sludge

\begin{tabular}{|c|c|c|c|c|c|c|c|c|c|c|}
\hline Analyte (Method) & Units & $\begin{array}{c}\text { 1st } \\
\text { Replicate } \\
\end{array}$ & $\begin{array}{c}\text { 2nd } \\
\text { Replicate } \\
\end{array}$ & $\begin{array}{c}\text { 3rd } \\
\text { Replicate } \\
\end{array}$ & $\begin{array}{c}\text { 4th } \\
\text { Replicate } \\
\end{array}$ & Average & \%RSD & Blank & $\begin{array}{c}\text { Average } \\
\text { Analyzed } \\
\text { Glass Std }\end{array}$ & $\begin{array}{c}\text { Glass Std } \\
\text { Composition }\end{array}$ \\
\hline Ag (IE) & $\mathrm{mg} / \mathrm{kg}$ & $<2.7 \mathrm{E}+02$ & $<2.7 \mathrm{E}+02$ & $<2.7 \mathrm{E}+02$ & $<2.6 \mathrm{E}+02$ & - & - & $<2.9 \mathrm{E}-01$ & $<3.5 \mathrm{E}+02$ & - \\
\hline $\mathrm{Al}$ (IE) & $\mathrm{mg} / \mathrm{kg}$ & $7.98 \mathrm{E}+04$ & $7.47 \mathrm{E}+04$ & $8.72 E+04$ & $7.25 E+04$ & $7.86 E+04$ & $8.3 \%$ & $<1.0 \mathrm{E}+00$ & $2.56 \mathrm{E}+04$ & $2.50 \mathrm{E}+04$ \\
\hline B (IE) & $\mathrm{mg} / \mathrm{kg}$ & $<6.9 \mathrm{E}+02$ & $<7.0 \mathrm{E}+02$ & $<6.8 \mathrm{E}+02$ & $<6.8 \mathrm{E}+02$ & - & - & $<7.5 \mathrm{E}-01$ & $2.70 \mathrm{E}+04$ & $2.69 \mathrm{E}+04$ \\
\hline $\mathrm{Ba}$ (IE) & $\mathrm{mg} / \mathrm{kg}$ & $3.35 \mathrm{E}+02$ & $3.34 \mathrm{E}+02$ & $3.46 \mathrm{E}+02$ & $3.44 \mathrm{E}+02$ & $3.40 \mathrm{E}+02$ & $1.8 \%$ & $<6.3 \mathrm{E}-02$ & $8.69 \mathrm{E}+02$ & $7.90 \mathrm{E}+02$ \\
\hline Be (IE) & $\mathrm{mg} / \mathrm{kg}$ & $<6.0 \mathrm{E}+00$ & $<6.0 \mathrm{E}+00$ & $<5.9 \mathrm{E}+00$ & $<5.8 \mathrm{E}+00$ & - & - & $<6.4 \mathrm{E}-03$ & $<7.8 \mathrm{E}+00$ & - \\
\hline Ca (IE) & $\mathrm{mg} / \mathrm{kg}$ & $5.93 \mathrm{E}+03$ & $6.01 \mathrm{E}+03$ & $5.99 \mathrm{E}+03$ & $6.24 \mathrm{E}+03$ & $6.04 E+03$ & $2.3 \%$ & $<9.8 \mathrm{E}-01$ & $1.10 \mathrm{E}+04$ & $1.02 \mathrm{E}+04$ \\
\hline Cd (IE) & $\mathrm{mg} / \mathrm{kg}$ & $1.85 \mathrm{E}+02$ & $2.02 E+02$ & $1.98 \mathrm{E}+02$ & $2.02 \mathrm{E}+02$ & $1.97 E+02$ & $4.1 \%$ & $<8.2 \mathrm{E}-02$ & $<1.0 \mathrm{E}+02$ & - \\
\hline Ce (IE) & $\mathrm{mg} / \mathrm{kg}$ & $<5.3 \mathrm{E}+03$ & $<5.3 \mathrm{E}+03$ & $<5.2 \mathrm{E}+03$ & $<5.2 \mathrm{E}+03$ & - & - & $<5.7 \mathrm{E}+00$ & $<6.9 \mathrm{E}+03$ & - \\
\hline Cr (IE) & $\mathrm{mg} / \mathrm{kg}$ & $2.58 \mathrm{E}+02$ & $2.13 E+02$ & $2.24 \mathrm{E}+02$ & $2.24 \mathrm{E}+02$ & $2.30 \mathrm{E}+02$ & $8.5 \%$ & $<1.9 \mathrm{E}-01$ & $6.93 \mathrm{E}+02$ & $6.40 \mathrm{E}+02$ \\
\hline $\mathrm{Cu}$ (IE) & $\mathrm{mg} / \mathrm{kg}$ & $<2.9 \mathrm{E}+02$ & $<2.9 \mathrm{E}+02$ & $<2.9 \mathrm{E}+02$ & $<2.8 \mathrm{E}+02$ & - & - & $<3.1 \mathrm{E}-01$ & $<3.2 \mathrm{E}+02$ & $3.00 \mathrm{E}+01$ \\
\hline Fe (IE) & $\mathrm{mg} / \mathrm{kg}$ & $5.26 \mathrm{E}+04$ & $5.25 \mathrm{E}+04$ & $5.30 \mathrm{E}+04$ & $5.36 \mathrm{E}+04$ & $5.29 \mathrm{E}+04$ & $0.9 \%$ & $<2.8 \mathrm{E}-01$ & $1.02 \mathrm{E}+05$ & $9.79 \mathrm{E}+04$ \\
\hline Gd (IE) & $\mathrm{mg} / \mathrm{kg}$ & $<3.2 \mathrm{E}+02$ & $<3.3 \mathrm{E}+02$ & $<3.2 \mathrm{E}+02$ & $<3.2 \mathrm{E}+02$ & - & - & $<3.5 \mathrm{E}-01$ & $<4.2 \mathrm{E}+02$ & - \\
\hline K (IE) & $\mathrm{mg} / \mathrm{kg}$ & $<4.4 \mathrm{E}+03$ & $<4.5 \mathrm{E}+03$ & $<4.4 \mathrm{E}+03$ & $<4.3 \mathrm{E}+03$ & - & - & $<4.8 \mathrm{E}+00$ & $2.26 \mathrm{E}+04$ & $2.26 \mathrm{E}+04$ \\
\hline La (IE) & $\mathrm{mg} / \mathrm{kg}$ & $<3.9 \mathrm{E}+02$ & $<4.0 \mathrm{E}+02$ & $<3.9 \mathrm{E}+02$ & $<3.9 \mathrm{E}+02$ & - & - & $<4.3 \mathrm{E}-01$ & $<5.2 \mathrm{E}+02$ & - \\
\hline Li (IE) & $\mathrm{mg} / \mathrm{kg}$ & $<2.7 \mathrm{E}+02$ & $<2.8 \mathrm{E}+02$ & $<2.7 \mathrm{E}+02$ & $<2.7 \mathrm{E}+02$ & - & - & $<2.9 \mathrm{E}-01$ & $1.44 \mathrm{E}+04$ & $1.49 \mathrm{E}+04$ \\
\hline Mg (IE) & $\mathrm{mg} / \mathrm{kg}$ & $2.23 E+03$ & $2.24 \mathrm{E}+03$ & $2.27 \mathrm{E}+03$ & $2.30 \mathrm{E}+03$ & $2.26 \mathrm{E}+03$ & $1.4 \%$ & 5.57E-02 & $5.46 \mathrm{E}+03$ & $5.20 \mathrm{E}+03$ \\
\hline $\mathrm{Mn}$ (IE) & $\mathrm{mg} / \mathrm{kg}$ & $1.26 \mathrm{E}+04$ & $1.27 \mathrm{E}+04$ & $1.28 \mathrm{E}+04$ & $1.30 \mathrm{E}+04$ & $1.28 \mathrm{E}+04$ & $1.3 \%$ & $<2.2 \mathrm{E}-02$ & $1.51 \mathrm{E}+04$ & $1.46 \mathrm{E}+04$ \\
\hline Mo (IE) & $\mathrm{mg} / \mathrm{kg}$ & $<2.9 \mathrm{E}+02$ & $<3.0 \mathrm{E}+02$ & $<2.9 \mathrm{E}+02$ & $<2.9 \mathrm{E}+02$ & - & - & $<3.2 \mathrm{E}-01$ & $<3.9 \mathrm{E}+02$ & - \\
\hline $\mathrm{Na}$ (IE) & $\mathrm{mg} / \mathrm{kg}$ & $3.48 \mathrm{E}+05$ & $3.51 \mathrm{E}+05$ & $3.56 \mathrm{E}+05$ & $3.59 E+05$ & $3.54 \mathrm{E}+05$ & $1.4 \%$ & $<2.8 \mathrm{E}+00$ & $8.95 \mathrm{E}+04$ & $8.52 \mathrm{E}+04$ \\
\hline $\mathrm{Ni}$ (IE) & $\mathrm{mg} / \mathrm{kg}$ & $4.39 \mathrm{E}+03$ & $4.35 E+03$ & $4.43 \mathrm{E}+03$ & $4.46 \mathrm{E}+03$ & $4.41 E+03$ & $1.1 \%$ & $<5.1 \mathrm{E}-01$ & $8.25 \mathrm{E}+03$ & $8.27 \mathrm{E}+03$ \\
\hline
\end{tabular}

Divide $\mathrm{mg} / \mathrm{kg}$ values by $1 \mathrm{E}+04$ to convert to wt \% dried solids basis 
WSRC-STI-2007-00697, REV. 0

Table 3-9. Composition of the Aqua Regia Dissolution of Total Dried Solids from the Post Aluminum Dissolution Sludge (Continued)

\begin{tabular}{|c|c|c|c|c|c|c|c|c|c|c|}
\hline Analyte (Method) & Units & $\begin{array}{c}\text { 1st } \\
\text { Replicate } \\
\end{array}$ & $\begin{array}{c}\text { 2nd } \\
\text { Replicate } \\
\end{array}$ & $\begin{array}{c}\text { 3rd } \\
\text { Replicate } \\
\end{array}$ & $\begin{array}{c}\text { 4th } \\
\text { Replicate } \\
\end{array}$ & Average & \%RSD & Blank & $\begin{array}{c}\text { Average } \\
\text { Analyzed } \\
\text { Glass Std }\end{array}$ & $\begin{array}{c}\text { Glass Std } \\
\text { Composition }\end{array}$ \\
\hline $\mathrm{P}$ (IE) & $\mathrm{mg} / \mathrm{kg}$ & $1.40 \mathrm{E}+03$ & $1.33 \mathrm{E}+03$ & $1.40 \mathrm{E}+03$ & $<1.33 \mathrm{e} 3$ & $1.38 \mathrm{E}+03^{*}$ & $2.9 \%$ & $<1.5 \mathrm{E}+00$ & $1.70 \mathrm{E}+03$ & $1.10 \mathrm{E}+03$ \\
\hline $\mathrm{Pb}$ (IE) & $\mathrm{mg} / \mathrm{kg}$ & $<7.6 \mathrm{E}+02$ & $<7.7 \mathrm{E}+02$ & $<7.5 \mathrm{E}+02$ & $<7.5 \mathrm{E}+02$ & - & - & $<8.3 \mathrm{E}-01$ & $<1.0 \mathrm{E}+03$ & - \\
\hline S (IE) & $\mathrm{mg} / \mathrm{kg}$ & $<1.1 \mathrm{E}+04$ & $<1.1 \mathrm{E}+04$ & $<1.1 \mathrm{E}+04$ & $<1.1 \mathrm{E}+04$ & - & - & $<1.2 \mathrm{E}+01$ & $<1.4 \mathrm{E}+04$ & - \\
\hline Sb (IE) & $\mathrm{mg} / \mathrm{kg}$ & $<1.5 \mathrm{E}+03$ & $<1.5 \mathrm{E}+03$ & $<1.4 \mathrm{E}+03$ & $<1.4 \mathrm{E}+03$ & - & - & $<1.6 \mathrm{E}+00$ & $<1.9 \mathrm{E}+03$ & - \\
\hline Sn (IE) & $\mathrm{mg} / \mathrm{kg}$ & $<4.4 \mathrm{E}+03$ & $<4.4 \mathrm{E}+03$ & $<4.3 \mathrm{E}+03$ & $<4.3 \mathrm{E}+03$ & - & - & $<4.7 \mathrm{E}+00$ & $<5.7 \mathrm{E}+03$ & - \\
\hline Sr (IE) & $\mathrm{mg} / \mathrm{kg}$ & $<3.9 \mathrm{E}+02$ & $<3.9 \mathrm{E}+02$ & $<3.8 \mathrm{E}+02$ & $<3.8 \mathrm{E}+02$ & - & - & $<4.2 \mathrm{E}-01$ & $<4.3 \mathrm{E}+02$ & $3.00 \mathrm{E}+01$ \\
\hline Ti (IE) & $\mathrm{mg} / \mathrm{kg}$ & $<1.7 \mathrm{E}+02$ & $<1.8 \mathrm{E}+02$ & $<1.7 \mathrm{E}+02$ & $<1.7 \mathrm{E}+02$ & - & - & $<1.9 \mathrm{E}-01$ & $6.49 \mathrm{E}+03$ & $6.90 \mathrm{E}+03$ \\
\hline U (IE) & $\mathrm{mg} / \mathrm{kg}$ & $<1.8 \mathrm{E}+04$ & $<1.8 \mathrm{E}+04$ & $<1.7 \mathrm{E}+04$ & $<1.7 \mathrm{E}+04$ & - & - & $<1.9 \mathrm{E}+01$ & $<2.3 \mathrm{E}+04$ & - \\
\hline V (IE) & $\mathrm{mg} / \mathrm{kg}$ & $<7.3 \mathrm{E}+01$ & $<7.4 \mathrm{E}+01$ & $<7.2 \mathrm{E}+01$ & $<7.1 \mathrm{E}+01$ & - & - & $<7.9 \mathrm{E}-02$ & $<9.6 \mathrm{E}+01$ & - \\
\hline $\mathrm{Zn}$ (IE) & $\mathrm{mg} / \mathrm{kg}$ & $<9.5 \mathrm{E}+02$ & $<9.6 \mathrm{E}+02$ & $<9.4 \mathrm{E}+02$ & $<9.3 \mathrm{E}+02$ & - & - & $<1.0 \mathrm{E}+00$ & $<1.1 \mathrm{E}+03$ & $1.60 \mathrm{E}+02$ \\
\hline Zr (IE) & $\mathrm{mg} / \mathrm{kg}$ & $7.77 \mathrm{E}+02$ & $7.79 \mathrm{E}+02$ & $7.93 E+02$ & $2.50 \mathrm{E}+02$ & $6.50 \mathrm{E}+02$ & $41 \%$ & $<2.0 \mathrm{E}-01$ & $6.25 \mathrm{E}+02$ & $9.60 \mathrm{E}+02$ \\
\hline $\mathrm{Hg}(\mathrm{CV})$ & $\mathrm{mg} / \mathrm{kg}$ & $1.46 \mathrm{E}+04$ & $1.47 \mathrm{E}+04$ & $1.43 E+04$ & $1.44 \mathrm{E}+04$ & $1.45 E+04$ & $1.3 \%$ & $<0.11$ & - & - \\
\hline
\end{tabular}

* - Indicate that not all replicates were used to calculate the Average and the \%RSD.

Divide $\mathrm{mg} / \mathrm{kg}$ values by $1 \mathrm{E}+04$ to convert to wt \% dried solids basis 
WSRC-STI-2007-00697, REV. 0

Table 3-10. Composition of the Peroxide Fusion Dissolution of Total Dried Solids from the Post Aluminum Dissolution Sludge

\begin{tabular}{|c|c|c|c|c|c|c|c|c|c|c|}
\hline Analyte (Method) & Units & $\begin{array}{c}\text { 1st } \\
\text { Replicate }\end{array}$ & $\begin{array}{c}\text { 2nd } \\
\text { Replicate }\end{array}$ & $\begin{array}{c}\text { 3rd } \\
\text { Replicate }\end{array}$ & $\begin{array}{c}\text { 4th } \\
\text { Replicate }\end{array}$ & Average & $\%$ RSD & Blank & $\begin{array}{c}\text { Average } \\
\text { Analyzed } \\
\text { Glass Std }\end{array}$ & $\begin{array}{c}\text { Glass Std } \\
\text { Composition }\end{array}$ \\
\hline Ag (IE) & $\mathrm{mg} / \mathrm{kg}$ & $<8.0 \mathrm{E}+02$ & $<7.9 \mathrm{E}+02$ & $<8.5 \mathrm{E}+02$ & $<8.4 \mathrm{E}+02$ & - & - & $<9.6 \mathrm{E}-01$ & $<9.6 \mathrm{E}+02$ & - \\
\hline $\mathrm{Al}$ (IE) & $\mathrm{mg} / \mathrm{kg}$ & $9.09 \mathrm{E}+04$ & $9.10 \mathrm{E}+04$ & $8.76 \mathrm{E}+04$ & $8.72 E+04$ & $8.92 E+04$ & $2.3 \%$ & $<1.0 \mathrm{E}+00$ & $2.66 \mathrm{E}+04$ & $2.50 \mathrm{E}+04$ \\
\hline B (IE) & $\mathrm{mg} / \mathrm{kg}$ & $<6.2 \mathrm{E}+02$ & $<6.1 \mathrm{E}+02$ & $<6.6 \mathrm{E}+02$ & $<6.6 \mathrm{E}+02$ & - & - & $<7.5 \mathrm{E}-01$ & $2.66 \mathrm{E}+04$ & $2.69 \mathrm{E}+04$ \\
\hline $\mathrm{Ba}$ (IE) & $\mathrm{mg} / \mathrm{kg}$ & $3.42 \mathrm{E}+02$ & $3.41 \mathrm{E}+02$ & $3.27 \mathrm{E}+02$ & $3.22 \mathrm{E}+02$ & $3.33 E+02$ & $3.0 \%$ & $<6.3 \mathrm{E}-02$ & $8.47 \mathrm{E}+02$ & $7.90 \mathrm{E}+02$ \\
\hline Be (IE) & $\mathrm{mg} / \mathrm{kg}$ & $<5.4 \mathrm{E}+00$ & $<5.3 \mathrm{E}+00$ & $<5.7 \mathrm{E}+00$ & $<5.7 \mathrm{E}+00$ & - & - & $<6.4 \mathrm{E}-03$ & $<6.4 \mathrm{E}+00$ & - \\
\hline Ca (IE) & $\mathrm{mg} / \mathrm{kg}$ & $7.22 \mathrm{E}+03$ & $7.01 \mathrm{E}+03$ & $7.69 \mathrm{E}+03$ & $6.74 \mathrm{E}+03$ & $7.17 E+03$ & $5.6 \%$ & $1.18 \mathrm{E}+00$ & $1.25 \mathrm{E}+04$ & $1.02 \mathrm{E}+04$ \\
\hline Cd (IE) & $\mathrm{mg} / \mathrm{kg}$ & $1.88 \mathrm{E}+02$ & $2.00 \mathrm{E}+02$ & $1.72 \mathrm{E}+02$ & $1.82 \mathrm{E}+02$ & $1.86 \mathrm{E}+02$ & $6.3 \%$ & $<8.2 \mathrm{E}-02$ & $<8.2 \mathrm{E}+01$ & - \\
\hline Ce (IE) & $\mathrm{mg} / \mathrm{kg}$ & $<3.2 \mathrm{E}+03$ & $<3.1 \mathrm{E}+03$ & $<3.3 \mathrm{E}+03$ & $<3.3 \mathrm{E}+03$ & - & - & $<3.8 \mathrm{E}+00$ & $<3.8 \mathrm{E}+03$ & - \\
\hline Cr (IE) & $\mathrm{mg} / \mathrm{kg}$ & $2.93 \mathrm{E}+02$ & $2.45 E+02$ & $2.35 \mathrm{E}+02$ & $2.68 \mathrm{E}+02$ & $2.60 \mathrm{E}+02$ & $9.9 \%$ & $<1.9 \mathrm{E}-01$ & $6.67 \mathrm{E}+02$ & $6.40 \mathrm{E}+02$ \\
\hline $\mathrm{Cu}$ (IE) & $\mathrm{mg} / \mathrm{kg}$ & $2.33 \mathrm{E}+02$ & $2.16 \mathrm{E}+02$ & $<2.3 \mathrm{E}+02$ & $2.21 \mathrm{E}+02$ & $2.23 \mathrm{E}+02 *$ & $3.9 \%$ & $<2.6 \mathrm{E}-01$ & $<2.6 \mathrm{E}+02$ & $3.00 \mathrm{E}+01$ \\
\hline Fe (IE) & $\mathrm{mg} / \mathrm{kg}$ & $5.34 \mathrm{E}+04$ & $5.35 E+04$ & $5.14 \mathrm{E}+04$ & $5.11 \mathrm{E}+04$ & $5.24 \mathrm{E}+04$ & $2.4 \%$ & $<1.6 \mathrm{E}-01$ & $1.01 \mathrm{E}+05$ & $9.79 E+04$ \\
\hline Gd (IE) & $\mathrm{mg} / \mathrm{kg}$ & $<2.9 \mathrm{E}+02$ & $<2.9 \mathrm{E}+02$ & $<3.1 \mathrm{E}+02$ & $<3.1 \mathrm{E}+02$ & - & - & $<3.5 \mathrm{E}-01$ & $<3.5 \mathrm{E}+02$ & - \\
\hline K (IE) & $\mathrm{mg} / \mathrm{kg}$ & $<4.0 \mathrm{E}+03$ & $<3.9 \mathrm{E}+03$ & $<4.3 \mathrm{E}+03$ & $<4.2 \mathrm{E}+03$ & - & - & $<4.8 \mathrm{E}+00$ & $2.53 \mathrm{E}+04$ & $2.26 \mathrm{E}+04$ \\
\hline La (IE) & $\mathrm{mg} / \mathrm{kg}$ & $<3.6 \mathrm{E}+02$ & $<3.5 \mathrm{E}+02$ & $<3.8 \mathrm{E}+02$ & $<3.7 \mathrm{E}+02$ & - & - & $<4.3 \mathrm{E}-01$ & $<4.3 \mathrm{E}+02$ & - \\
\hline Li (IE) & $\mathrm{mg} / \mathrm{kg}$ & $<2.5 \mathrm{E}+02$ & $<2.4 \mathrm{E}+02$ & $<2.6 \mathrm{E}+02$ & $<2.6 \mathrm{E}+02$ & - & - & $<2.9 \mathrm{E}-01$ & $1.42 \mathrm{E}+04$ & $1.49 \mathrm{E}+04$ \\
\hline Mg (IE) & $\mathrm{mg} / \mathrm{kg}$ & $2.23 E+03$ & $2.28 \mathrm{E}+03$ & $2.09 \mathrm{E}+03$ & $2.18 \mathrm{E}+03$ & $2.20 \mathrm{E}+03$ & $3.7 \%$ & $<3.5 \mathrm{E}-02$ & $5.40 \mathrm{E}+03$ & $5.20 \mathrm{E}+03$ \\
\hline $\mathrm{Mn}$ (IE) & $\mathrm{mg} / \mathrm{kg}$ & $1.28 \mathrm{E}+04$ & $1.29 \mathrm{E}+04$ & $1.23 \mathrm{E}+04$ & $1.24 \mathrm{E}+04$ & $1.26 E+04$ & $2.3 \%$ & $<1.6 \mathrm{E}-02$ & $1.47 \mathrm{E}+04$ & $1.46 \mathrm{E}+04$ \\
\hline Mo (IE) & $\mathrm{mg} / \mathrm{kg}$ & $<2.7 \mathrm{E}+02$ & $<2.6 \mathrm{E}+02$ & $<2.8 \mathrm{E}+02$ & $<2.8 \mathrm{E}+02$ & - & - & $<3.2 \mathrm{E}-01$ & $<3.2 \mathrm{E}+02$ & - \\
\hline $\mathrm{Ni}$ (IE) & $\mathrm{mg} / \mathrm{kg}$ & $4.36 \mathrm{E}+03$ & $4.33 E+03$ & $4.18 \mathrm{E}+03$ & $4.14 \mathrm{E}+03$ & $4.25 E+03$ & $2.6 \%$ & $<5.1 \mathrm{E}-01$ & $8.32 \mathrm{E}+03$ & $8.27 \mathrm{E}+03$ \\
\hline
\end{tabular}

* - Indicate that not all replicates were used to calculate the Average and the \%RSD.

Divide $\mathrm{mg} / \mathrm{kg}$ values by $1 \mathrm{E}+04$ to convert to wt $\%$ dried solids basis 
WSRC-STI-2007-00697, REV. 0

Table 3-10. Composition of the Peroxide Fusion Dissolution of Total Dried Solids from the Post Aluminum Dissolution Sludge (Continued)

\begin{tabular}{|c|c|c|c|c|c|c|c|c|c|c|}
\hline Analyte (Method) & Units & $\begin{array}{c}\text { 1st } \\
\text { Replicate } \\
\end{array}$ & $\begin{array}{c}\text { 2nd } \\
\text { Replicate }\end{array}$ & $\begin{array}{c}\text { 3rd } \\
\text { Replicate } \\
\end{array}$ & $\begin{array}{c}\text { 4th } \\
\text { Replicate }\end{array}$ & Average & $\%$ RSD & Blank & $\begin{array}{c}\text { Average } \\
\text { Analyzed } \\
\text { Glass Std }\end{array}$ & $\begin{array}{c}\text { Glass Std } \\
\text { Composition }\end{array}$ \\
\hline $\mathrm{P}$ (IE) & $\mathrm{mg} / \mathrm{kg}$ & $<1.2 \mathrm{E}+03$ & $<1.2 \mathrm{E}+03$ & $<1.3 \mathrm{E}+03$ & $<1.3 \mathrm{E}+03$ & - & - & $<1.5 \mathrm{E}+00$ & $<1.5 \mathrm{E}+03$ & $1.10 \mathrm{E}+03$ \\
\hline $\mathrm{Pb}$ (IE) & $\mathrm{mg} / \mathrm{kg}$ & $<6.9 \mathrm{E}+02$ & $<6.8 \mathrm{E}+02$ & $<7.3 \mathrm{E}+02$ & $<7.3 \mathrm{E}+02$ & - & - & $<8.3 \mathrm{E}-01$ & $<8.3 E+02$ & - \\
\hline S (IE) & $\mathrm{mg} / \mathrm{kg}$ & $<9.9 \mathrm{E}+03$ & $<9.7 \mathrm{E}+03$ & $<1.1 \mathrm{E}+04$ & $<1.0 \mathrm{E}+04$ & - & - & $<1.2 \mathrm{E}+01$ & $<1.2 \mathrm{E}+04$ & - \\
\hline Sb (IE) & $\mathrm{mg} / \mathrm{kg}$ & $<1.3 \mathrm{E}+03$ & $<1.3 \mathrm{E}+03$ & $<1.4 \mathrm{E}+03$ & $<1.4 \mathrm{E}+03$ & - & - & $<1.6 \mathrm{E}+00$ & $<1.6 \mathrm{E}+03$ & - \\
\hline Si (IE) & $\mathrm{mg} / \mathrm{kg}$ & $3.08 \mathrm{E}+03$ & $3.24 \mathrm{E}+03$ & $3.00 \mathrm{E}+03$ & $3.04 \mathrm{E}+03$ & $3.09 E+03$ & $3.4 \%$ & $<2.6 \mathrm{E}+00$ & $2.26 \mathrm{E}+05$ & $2.24 \mathrm{E}+05$ \\
\hline Sn (IE) & $\mathrm{mg} / \mathrm{kg}$ & $<3.9 \mathrm{E}+03$ & $<3.9 \mathrm{E}+03$ & $<4.2 \mathrm{E}+03$ & $<4.1 \mathrm{E}+03$ & - & - & $<4.7 \mathrm{E}+00$ & $<4.7 \mathrm{E}+03$ & - \\
\hline Sr (IE) & $\mathrm{mg} / \mathrm{kg}$ & $<3.5 \mathrm{E}+02$ & $<3.4 \mathrm{E}+02$ & $<3.7 \mathrm{E}+02$ & $<3.7 \mathrm{E}+02$ & - & - & $<4.2 \mathrm{E}-01$ & $<4.2 \mathrm{E}+02$ & $3.00 \mathrm{E}+01$ \\
\hline Ti (IE) & $\mathrm{mg} / \mathrm{kg}$ & $<1.6 \mathrm{E}+02$ & $<1.5 \mathrm{E}+02$ & $<1.7 \mathrm{E}+02$ & $<1.7 \mathrm{E}+02$ & - & - & $<1.9 \mathrm{E}-01$ & $7.08 \mathrm{E}+03$ & $6.90 \mathrm{E}+03$ \\
\hline U (IE) & $\mathrm{mg} / \mathrm{kg}$ & $<1.6 \mathrm{E}+04$ & $<1.6 \mathrm{E}+04$ & $<1.7 \mathrm{E}+04$ & $<1.7 \mathrm{E}+04$ & - & - & $<1.9 \mathrm{E}+01$ & $<1.9 \mathrm{E}+04$ & - \\
\hline V (IE) & $\mathrm{mg} / \mathrm{kg}$ & $<6.6 \mathrm{E}+01$ & $<6.5 \mathrm{E}+01$ & $<7.0 \mathrm{E}+01$ & $<6.9 \mathrm{E}+01$ & - & - & $<7.9 \mathrm{E}-02$ & $<7.9 \mathrm{E}+01$ & - \\
\hline $\mathrm{Zn}$ (IE) & $\mathrm{mg} / \mathrm{kg}$ & $<8.6 \mathrm{E}+02$ & $<8.4 \mathrm{E}+02$ & $<9.1 \mathrm{E}+02$ & $<9.1 \mathrm{E}+02$ & - & - & $<1.0 \mathrm{E}+00$ & $<1.0 \mathrm{E}+03$ & $1.60 \mathrm{E}+02$ \\
\hline${ }^{233} \mathrm{U}$ (IM) & $\mathrm{mg} / \mathrm{kg}$ & $<1.9 \mathrm{E}+00$ & $<1.8 \mathrm{E}+00$ & $<2.0 \mathrm{E}+00$ & $<2.0 \mathrm{E}+00$ & & & $<1.9 \mathrm{E}+00$ & - & - \\
\hline${ }^{234} \mathrm{U}$ (IM) & $\mathrm{mg} / \mathrm{kg}$ & $4.91 \mathrm{E}+00$ & $4.99 \mathrm{E}+00$ & $5.14 \mathrm{E}+00$ & $5.59 \mathrm{E}+00$ & $5.16 \mathrm{E}+00$ & $5.9 \%$ & $<3.8 \mathrm{E}+00$ & - & - \\
\hline${ }^{235} \mathrm{U}$ (IM) & $\mathrm{mg} / \mathrm{kg}$ & $9.23 \mathrm{E}+01$ & $9.87 \mathrm{E}+01$ & $8.67 \mathrm{E}+01$ & $9.26 \mathrm{E}+01$ & $9.26 \mathrm{E}+01$ & $5.3 \%$ & $<1.9 \mathrm{E}+00$ & - & - \\
\hline${ }^{236} \mathrm{U}$ (IM) & $\mathrm{mg} / \mathrm{kg}$ & $8.06 \mathrm{E}+00$ & $8.01 \mathrm{E}+00$ & $8.16 \mathrm{E}+00$ & $8.03 E+00$ & 8.07E+00 & $0.8 \%$ & $<1.3 \mathrm{E}+00$ & - & - \\
\hline${ }^{238} \mathrm{U}$ (IM) & $\mathrm{mg} / \mathrm{kg}$ & $1.11 \mathrm{E}+04$ & $1.26 \mathrm{E}+04$ & $9.88 \mathrm{E}+03$ & $1.14 \mathrm{E}+04$ & $1.12 E+04$ & $10 \%$ & $<1.5 \mathrm{E}+02$ & - & - \\
\hline U total (IM) & $\mathrm{mg} / \mathrm{kg}$ & $1.12 \mathrm{E}+04$ & $1.27 \mathrm{E}+04$ & $9.98 \mathrm{E}+03$ & $1.15 E+04$ & $1.14 E+04$ & $9.9 \%$ & - & - & - \\
\hline
\end{tabular}

Divide $\mathrm{mg} / \mathrm{kg}$ values by $1 \mathrm{E}+04$ to convert to wt \% dried solids basis 
WSRC-STI-2007-00697, REV. 0

Table 3-10. Composition of the Peroxide Fusion Dissolution of Total Dried Solids from the Post Aluminum Dissolution Sludge (Continued)

\begin{tabular}{|c|c|c|c|c|c|c|c|c|c|c|}
\hline Analyte (Method) & Units & $\begin{array}{c}\text { 1st } \\
\text { Replicate } \\
\end{array}$ & $\begin{array}{c}\text { 2nd } \\
\text { Replicate } \\
\end{array}$ & $\begin{array}{c}\text { 3rd } \\
\text { Replicate } \\
\end{array}$ & $\begin{array}{c}\text { 4th } \\
\text { Replicate } \\
\end{array}$ & Average & \%RSD & Blank & $\begin{array}{c}\text { Average } \\
\text { Analyzed } \\
\text { Glass Std }\end{array}$ & $\begin{array}{c}\text { Glass Std } \\
\text { Composition }\end{array}$ \\
\hline${ }^{99}$ Tc (IM) & $\mathrm{mCi} / \mathrm{kg}$ & $1.06 \mathrm{E}-01$ & $9.74 \mathrm{E}-02$ & $1.07 \mathrm{E}-01$ & 8.08E-02 & 9.77E-02 & $12 \%$ & $<2.8 \mathrm{E}-02$ & - & - \\
\hline${ }^{237} \mathrm{~Np}$ (IM) & $\mathrm{mCi} / \mathrm{kg}$ & 5.89E-03 & $6.35 \mathrm{E}-03$ & 5.31E-03 & $5.41 \mathrm{E}-03$ & $5.74 \mathrm{E}-03$ & $8.3 \%$ & $<2.5 \mathrm{E}-03$ & - & - \\
\hline${ }^{239} \mathrm{Pu}$ (IM) & $\mathrm{mCi} / \mathrm{kg}$ & $3.57 \mathrm{E}+00$ & $4.03 E+00$ & $3.17 \mathrm{E}+00$ & $3.80 \mathrm{E}+00$ & $3.64 \mathrm{E}+00$ & $10 \%$ & $<2.6 \mathrm{E}+00$ & - & - \\
\hline${ }^{240} \mathrm{Pu}(\mathrm{IM})$ & $\mathrm{mCi} / \mathrm{kg}$ & $2.02 \mathrm{E}+00$ & $2.01 \mathrm{E}+00$ & $1.87 \mathrm{E}+00$ & $1.96 \mathrm{E}+00$ & $1.97 E+00$ & $3.3 \%$ & $<3.0 \mathrm{E}+01$ & - & - \\
\hline${ }^{242} \mathrm{Pu}(\mathrm{IM})$ & $\mathrm{mCi} / \mathrm{kg}$ & $<9.0 \mathrm{E}-03$ & $<8.8 \mathrm{E}-03$ & $<9.6 \mathrm{E}-03$ & $<9.5 \mathrm{E}-03$ & - & - & $<3.6 \mathrm{E}-01$ & - & - \\
\hline${ }^{238} \mathrm{Pu}$ (SA) & $\mathrm{mCi} / \mathrm{kg}$ & $2.16 \mathrm{E}+02$ & $2.25 E+02$ & $1.91 \mathrm{E}+02$ & $2.09 \mathrm{E}+02$ & $2.10 \mathrm{E}+02$ & $6.8 \%$ & $3.14 \mathrm{E}-05$ & - & - \\
\hline${ }^{239 / 240} \mathrm{Pu}(\mathrm{SA})$ & $\mathrm{mCi} / \mathrm{kg}$ & $5.63 \mathrm{E}+00$ & $6.44 \mathrm{E}+00$ & $5.36 \mathrm{E}+00$ & $5.81 \mathrm{E}+00$ & $5.81 \mathrm{E}+00$ & $7.9 \%$ & $<2.6 \mathrm{E}-05$ & - & - \\
\hline${ }^{241} \mathrm{Pu}$ (SA) & $\mathrm{mCi} / \mathrm{kg}$ & $7.16 \mathrm{E}+01$ & $7.25 E+01$ & $6.22 \mathrm{E}+01$ & $6.62 \mathrm{E}+01$ & $6.81 E+01$ & $7.1 \%$ & $<4.5 \mathrm{E}-04$ & - & - \\
\hline
\end{tabular}


Table 3-11. Post Aluminum Dissolution Sludge Settling Data

\begin{tabular}{|c|c|c|c|}
\hline Date and Time & $\begin{array}{c}\text { Elapsed Time } \\
\text { (hours) }\end{array}$ & $\begin{array}{c}\text { Settled Sludge } \\
\text { Volume } \\
\end{array}$ & $\begin{array}{c}\text { Percentage of Initial } \\
\text { Sludge Height }\end{array}$ \\
\hline 9/19/2007 9:30 & 0.0 & 2950 & $100 \%$ \\
\hline 9/19/2007 13:30 & 4.0 & 2600 & $88 \%$ \\
\hline 9/19/2007 14:45 & 5.3 & 2500 & $85 \%$ \\
\hline 9/20/2007 6:15 & 20.7 & 2250 & $76 \%$ \\
\hline 9/20/2007 12:30 & 27.0 & 2175 & $74 \%$ \\
\hline 9/21/2007 6:15 & 44.7 & 2025 & $69 \%$ \\
\hline 9/21/2007 11:00 & 49.5 & 2000 & $68 \%$ \\
\hline 9/24/2007 6:15 & 116.7 & 1750 & $59 \%$ \\
\hline 9/26/2007 6:15 & 164.7 & 1700 & $58 \%$ \\
\hline 9/27/2007 6:45 & 189.2 & 1650 & $56 \%$ \\
\hline 9/28/2007 10:10 & 216.7 & 1625 & $55 \%$ \\
\hline 10/1/2007 7:00 & 285.5 & 1600 & $54 \%$ \\
\hline 10/2/2007 6:05 & 308.6 & 1590 & $54 \%$ \\
\hline 10/5/2007 8:00 & 382.5 & 1590 & $54 \%$ \\
\hline 10/5/2007 12:00 & 386.5 & 1590 & $54 \%$ \\
\hline 10/5/2007 15:00 & 389.5 & 1590 & $54 \%$ \\
\hline 10/8/2007 8:00 & 454.5 & 1590 & $54 \%$ \\
\hline 10/8/2007 13:00 & 459.5 & 1590 & $54 \%$ \\
\hline 10/8/2007 15:30 & 462.0 & 1590 & $54 \%$ \\
\hline 10/9/2007 9:15 & 479.7 & 1590 & $54 \%$ \\
\hline 10/9/2007 16:30 & 487.0 & 1590 & $54 \%$ \\
\hline 10/10/2007 8:00 & 502.5 & 1590 & $54 \%$ \\
\hline
\end{tabular}


WSRC-STI-2007-00697, REV. 0

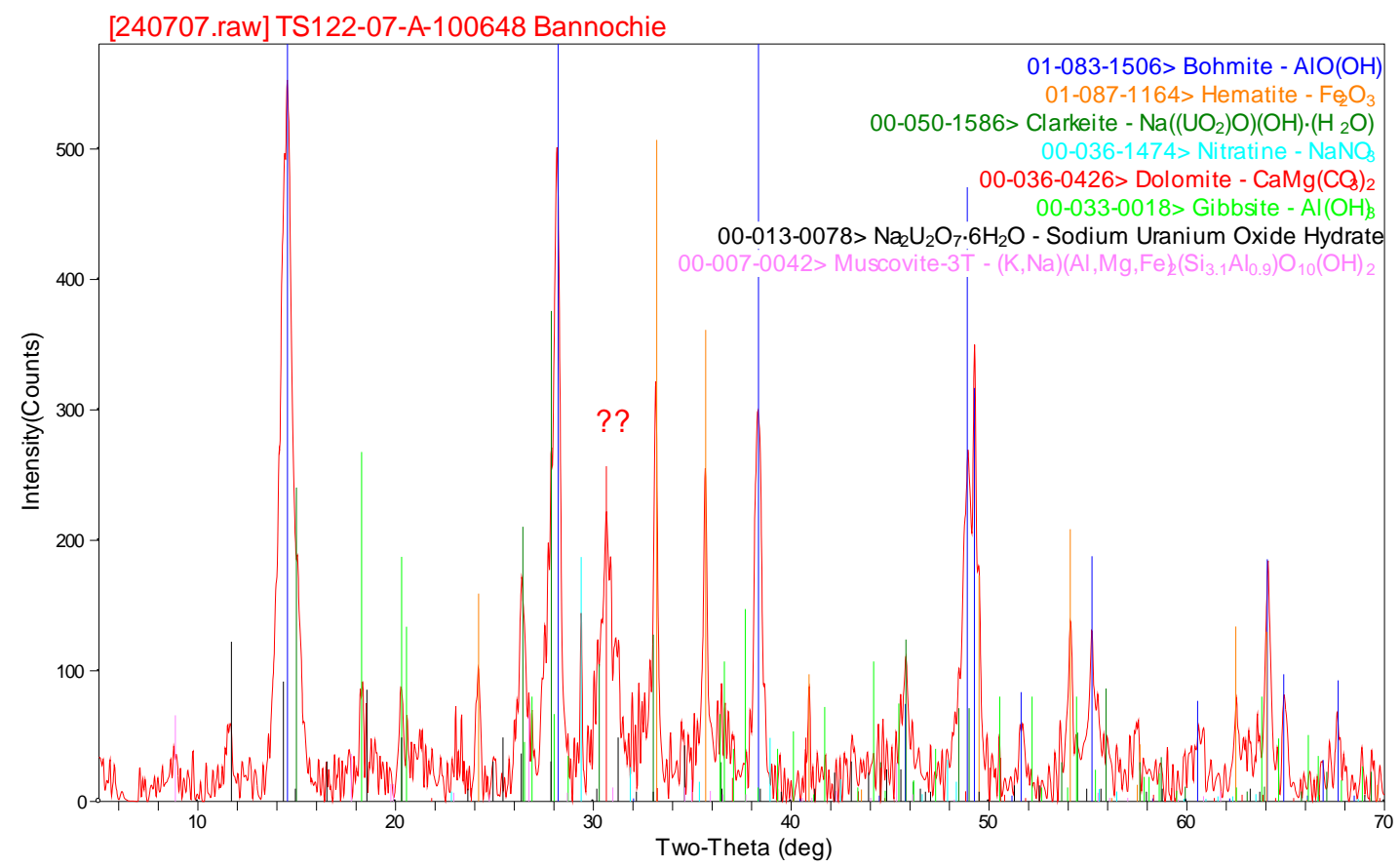

Figure 3.1 X-Ray Diffraction for the As-Received Tank 51H Sludge Solids

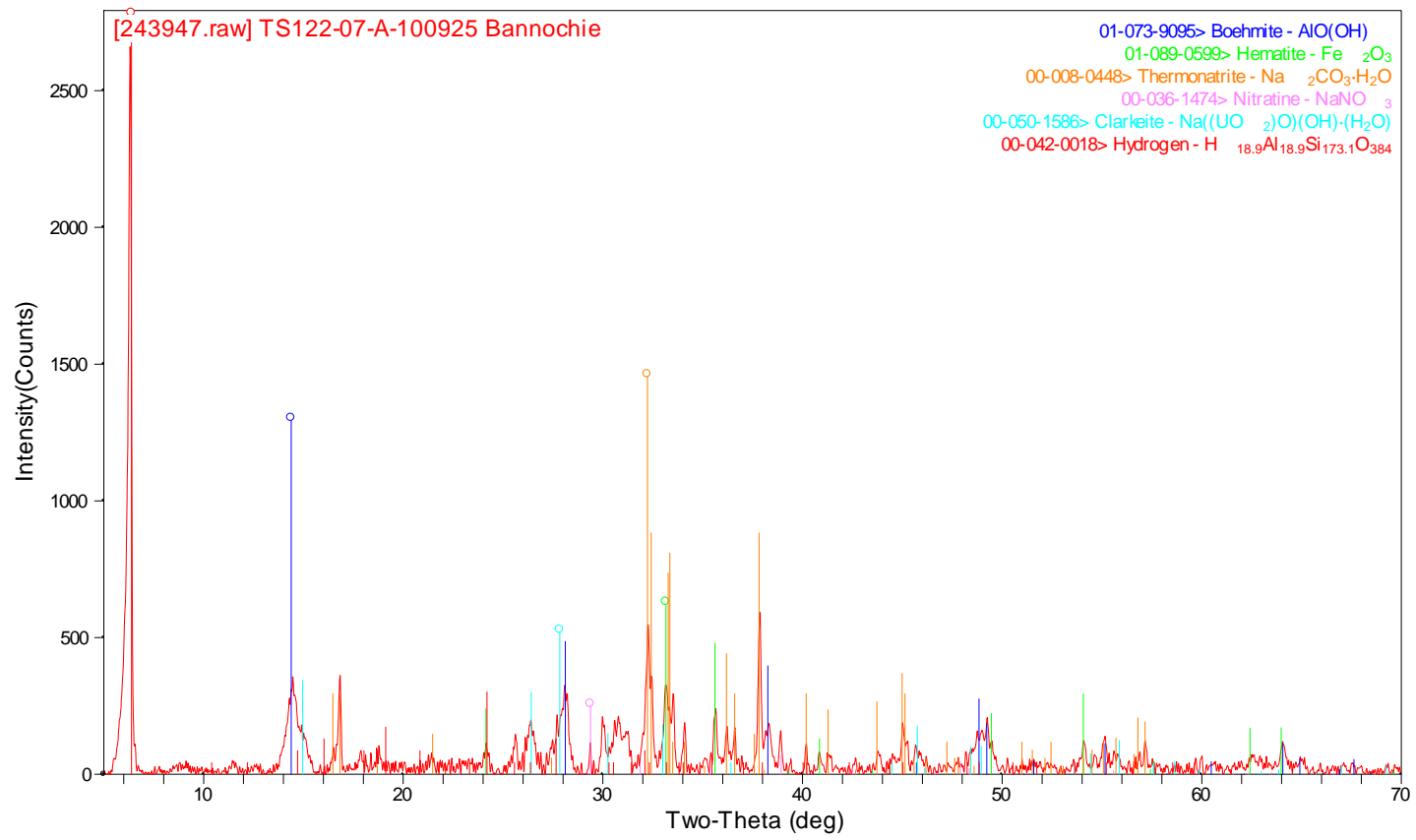

Figure 3.2 X-Ray Diffraction for Post Aluminum Dissolution Sludge Solids 


\subsection{DISCUSSION OF RESULTS}

\subsection{AS-RECEIVED TANK 5IH SLUDGE SAMPLE CHARACTERIZATION}

The characterization results for the as-received Tank 51H sludge sample (HTF-51-07-77) appear quite good with respect to the tight precision of the sample replicates and good results for the glass standards. Table 4-1 summarizes the composition of the key components of the sludge sample using the data from aqua regia and peroxide fusion dissolutions of the total dried solids in Tables 3-4 and 3-5. The supernate composition can be taken directly from Table 3-3.

Table 4-1. Summary of the As-Received Tank 51H Sludge Composition

\begin{tabular}{|c|c|c|c|}
\hline Analyte & Average & \%RSD & $\begin{array}{c}\text { No. of Values } \\
\text { Averaged }\end{array}$ \\
\hline Al mg/kg & $1.56 \mathrm{E}+05$ & $5.5 \%$ & $4^{\mathrm{d}}$ \\
\hline $\mathrm{Na} \mathrm{mg} / \mathrm{kg}$ & $1.45 \mathrm{E}+05$ & $3.3 \%$ & $4^{c}$ \\
\hline $\mathrm{Fe} \mathrm{mg} / \mathrm{kg}$ & $7.27 \mathrm{E}+04$ & $2.9 \%$ & 8 \\
\hline Mn mg/kg & $1.73 \mathrm{E}+04$ & $3.4 \%$ & 8 \\
\hline $\mathrm{Hg} \mathrm{mg} / \mathrm{kg}$ & $1.73 \mathrm{E}+04$ & $2.1 \%$ & $4^{\mathrm{c}}$ \\
\hline $\mathrm{U} \mathrm{mg} / \mathrm{kg}$ & $1.63 \mathrm{E}+04$ & $7.8 \%$ & $8^{\mathrm{a}}$ \\
\hline Ca mg/kg & $8.09 E+03$ & $6.2 \%$ & 8 \\
\hline $\mathrm{Ni} \mathrm{mg} / \mathrm{kg}$ & $6.46 \mathrm{E}+03$ & $2.8 \%$ & 8 \\
\hline Si mg/kg & $4.35 \mathrm{E}+03$ & $3.1 \%$ & $4^{\mathrm{d}}$ \\
\hline $\mathrm{Mg} \mathrm{mg/kg}$ & $2.95 \mathrm{E}+03$ & $4.2 \%$ & 8 \\
\hline${ }^{234} \mathrm{U} \mathrm{mg} / \mathrm{kg}$ & $7.83 \mathrm{E}+00$ & $22 \%$ & $4^{\mathrm{b}, \mathrm{d}}$ \\
\hline${ }^{235} \mathrm{U} \mathrm{mg} / \mathrm{kg}$ & $1.21 \mathrm{E}+02$ & $3.8 \%$ & $4^{\mathrm{b}, \mathrm{d}}$ \\
\hline${ }^{236} \mathrm{U} \mathrm{mg} / \mathrm{kg}$ & $1.13 \mathrm{E}+01$ & $14 \%$ & $4^{\mathrm{b}, \mathrm{d}}$ \\
\hline${ }^{238} \mathrm{U} \mathrm{mg} / \mathrm{kg}$ & $1.52 \mathrm{E}+04$ & $7.2 \%$ & $4^{\mathrm{b}, \mathrm{d}}$ \\
\hline${ }^{99} \mathrm{Tc} \quad \mathrm{mCi} / \mathrm{kg}$ & 9.35E-02 & $14 \%$ & $4^{\mathrm{d}}$ \\
\hline${ }^{237} \mathrm{~Np} \quad \mathrm{mCi} / \mathrm{kg}$ & 7.47E-03 & $5.0 \%$ & $4^{\mathrm{d}}$ \\
\hline${ }^{238} \mathrm{Pu} \quad \mathrm{mCi} / \mathrm{kg}$ & $3.17 \mathrm{E}+02$ & $13 \%$ & $4^{\mathrm{d}}$ \\
\hline${ }^{239 / 240} \mathrm{Pu} \quad \mathrm{mCi} / \mathrm{kg}$ & $7.54 \mathrm{E}+00$ & $13 \%$ & $8^{\mathrm{d}, \mathrm{e}}$ \\
\hline${ }^{241} \mathrm{Pu} \quad \mathrm{mCi} / \mathrm{kg}$ & $1.05 \mathrm{E}+02$ & $15 \%$ & $4^{\mathrm{d}}$ \\
\hline
\end{tabular}

Divide $\mathrm{mg} / \mathrm{kg}$ values by $1 \mathrm{E}+04$ to convert to wt \% dried solids basis

a Total uranium from averaging the combined values from ICP-ES and ICP-MS methods.

b Uranium isotopics obtained from averaging only ICP-MS. The sum of the uranium isotopes does not exactly match the total uranium since only a subset of the values could be used for the isotopics.

c Data taken from the aqua regia dissolution

d Data taken from the sodium peroxide fusion dissolution

e ${ }^{239 / 240} \mathrm{Pu}$ from averaging combined values from ICP-MS and Alpha Counting. 
WSRC-STI-2007-00697, REV. 0

\subsection{ALUMINUM DISOLUTION DEMONSTRATION RESULTS}

As shown from the results of the samples obtained during the demonstration (Table 3-6), the low temperature aluminum dissolution process appears to be effective at dissolving aluminum from the Tank $51 \mathrm{H}$ sludge. The process also seems to be highly selective for aluminum. The only other metal to dissolve to any appreciable extent was mercury based on the increased concentration of mercury in the decanted supernate at the conclusion of the aluminum dissolution demonstration. The mercury concentration in the supernate increased by a factor of $~ 10$ after the aluminum dissolution. The increase in the solution concentration of mercury indicates approximately $8 \%$ of the mercury in the sludge solids dissolved. The dissolution of a small amount of mercury during aluminum dissolution appears to be consistent with data collected during the In-Tank Sludge Processing Demonstration conducted in Tank $42 \mathrm{H}$ in $1982 .^{9}$

Figure 4.1 shows the increasing aluminum concentration in the supernate as a function of time during the aluminum dissolution demonstration. The graph shows the solution concentration of aluminum steadily increasing through the end of the three week test. The estimated percentage of aluminum dissolved also shown on the graph was calculated using a mass balance spreadsheet. The spreadsheet used the initial measured sludge composition, the actual amount of concentrated sodium hydroxide added, and the conversion of insoluble aluminum hydroxide to soluble sodium aluminate to determine the expected concentrations of aluminum and several other key species in the liquid and solid phase as a function of the percentage of aluminum dissolved. The balanced equation shown below was used to convert insoluble aluminum to a soluble form of aluminum in the spreadsheet.

$$
\mathrm{Al}(\mathrm{OH})_{3}+\mathrm{NaOH} \longrightarrow \mathrm{NaAlO}_{2}+2 \mathrm{H}_{2} \mathrm{O}
$$

Low temperature aluminum dissolution was envisioned as a process for dissolving the more easily digested Gibbsite form of aluminum, the assumption being that significantly higher temperatures would be necessary to dissolve any appreciable amount of the Boehmite form of aluminum. That may be an incorrect assumption as the rate of aluminum dissolution shown in Figure 4.1 closely follows the rate observed at Pacific Northwest National Laboratory (PNNL) with tests on pure samples of Boehmite conducted under similar conditions. ${ }^{10}$ However, the rate of aluminum dissolution can be influenced by several factors including particle size/contact surface area which may be significantly different between sludge solids and pure samples like those used in the PNNL work. More kinetic data on dissolution rates of the various forms of aluminum likely to be present in sludge solids would be required to draw firm conclusions.

The plot in Figure 4.1 shows that the aluminum concentration has not leveled out at the end of the test. This implies that more than $\sim 40 \%$ of the aluminum in the sludge could be dissolved given a longer reaction time. There is insufficient data to project the maximum amount of aluminum that could be dissolved under the current test conditions. 


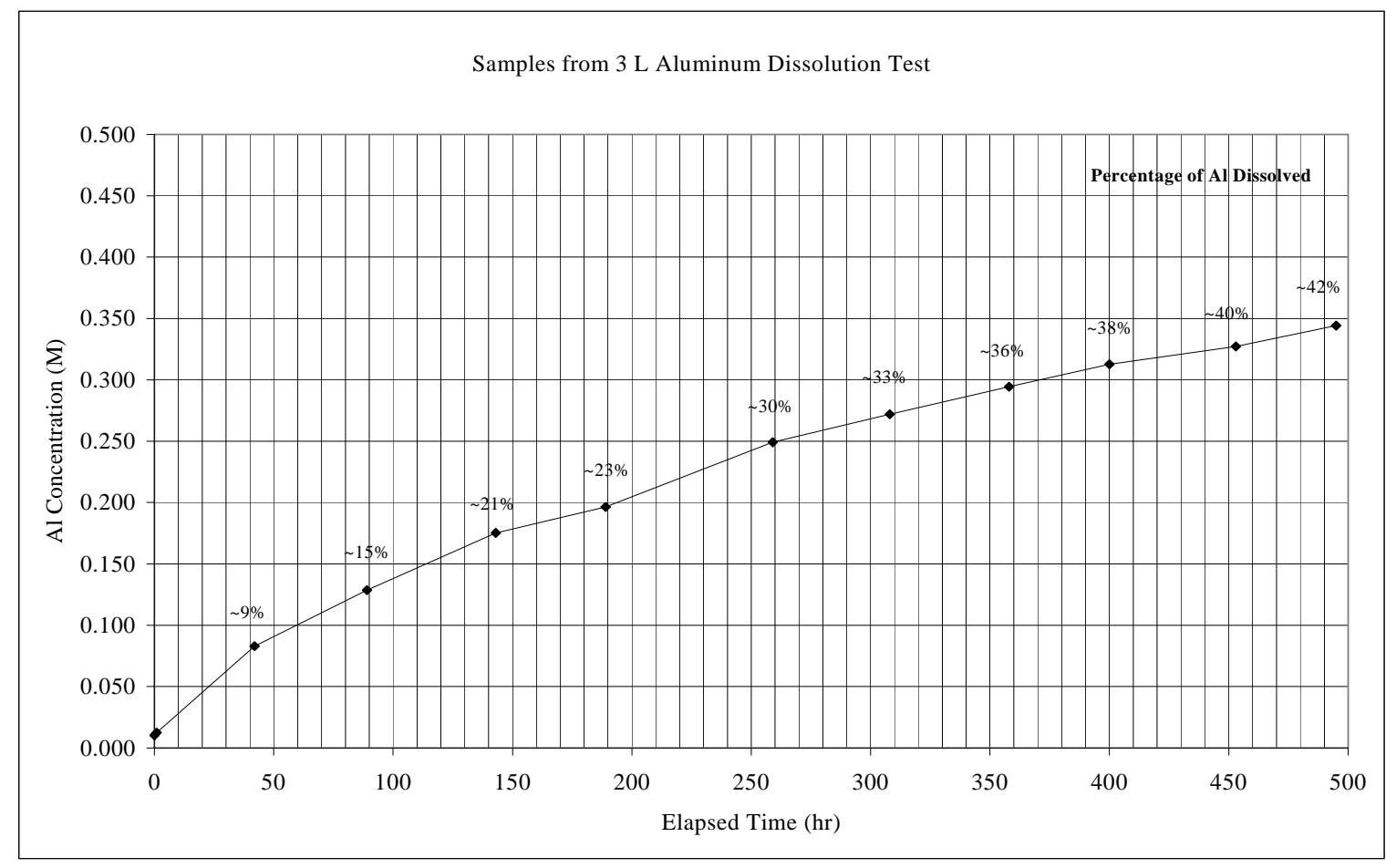

Figure 4.1 Aluminum Concentration in Solution during the Aluminum Dissolution Demonstration

\subsection{SLUDGE SETTLING AFTER ALUMINUM DISSOLUTION}

Figure 4.2 shows a plot of the post aluminum dissolution sludge settling data from Table 311. The sludge settled with a clear interface between the settling sludge solids and the clear supernate phase above. Although the sludge was allowed to settle for the prescribed three weeks, the sludge actually stopped settling after approximately 285 hours ( 12 days).

Since no settling data were obtained from the as-received Tank 51H sludge, a direct comparison between the settling characteristics of the as-received and post aluminum dissolution sludge cannot be made. However, Figure 4.3 shows settling data from an early sample of SB4 prior to washing. ${ }^{8}$ The earlier SB4 sludge settling rate varied significantly depending upon the size and material of the settling vessel. The two data sets shown in Figure 4.3 seem to be the most similar in terms of the material used for the settling vessel. The sludge height to vessel diameter ratio of the $1 \mathrm{~L}$ poly bottle and $250 \mathrm{~mL}$ poly cylinder were approximately 1 , while the ratio for the $3 \mathrm{~L}$ post aluminum dissolution sludge was approximately 1.5. From the flowsheet, the sludge height to vessel diameter ratio in Tank $51 \mathrm{H}$ after aluminum dissolution will be approximately 0.2. The graph in Figure 4.3 indicates minimal change in the settling characteristics of the sludge after aluminum dissolution. However, scaling these results to a large tank with a much lower sludge height to vessel diameter ratio would have high uncertainty. 


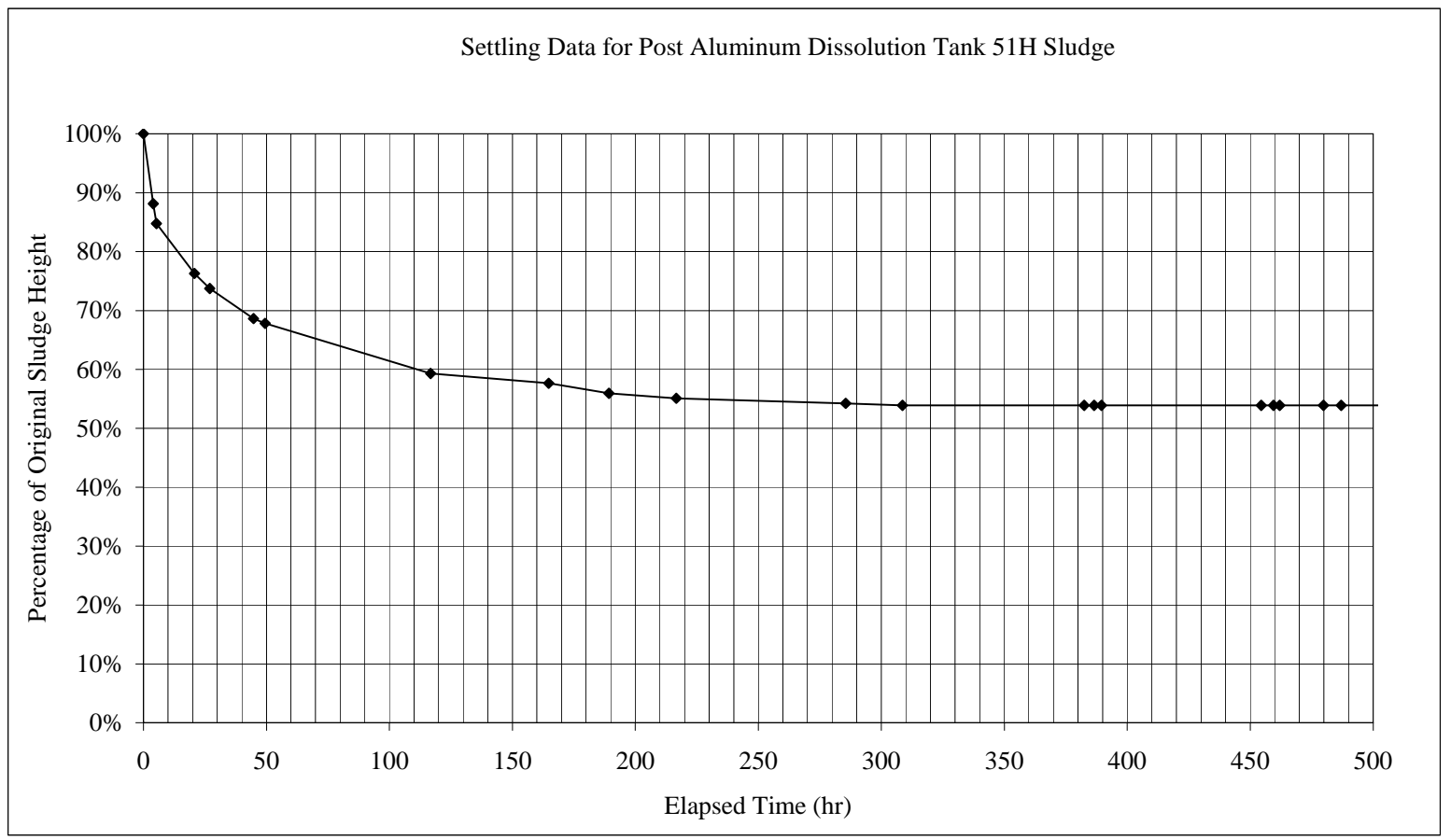

Figure 4.2 Post Aluminum Dissolution Sludge Settling

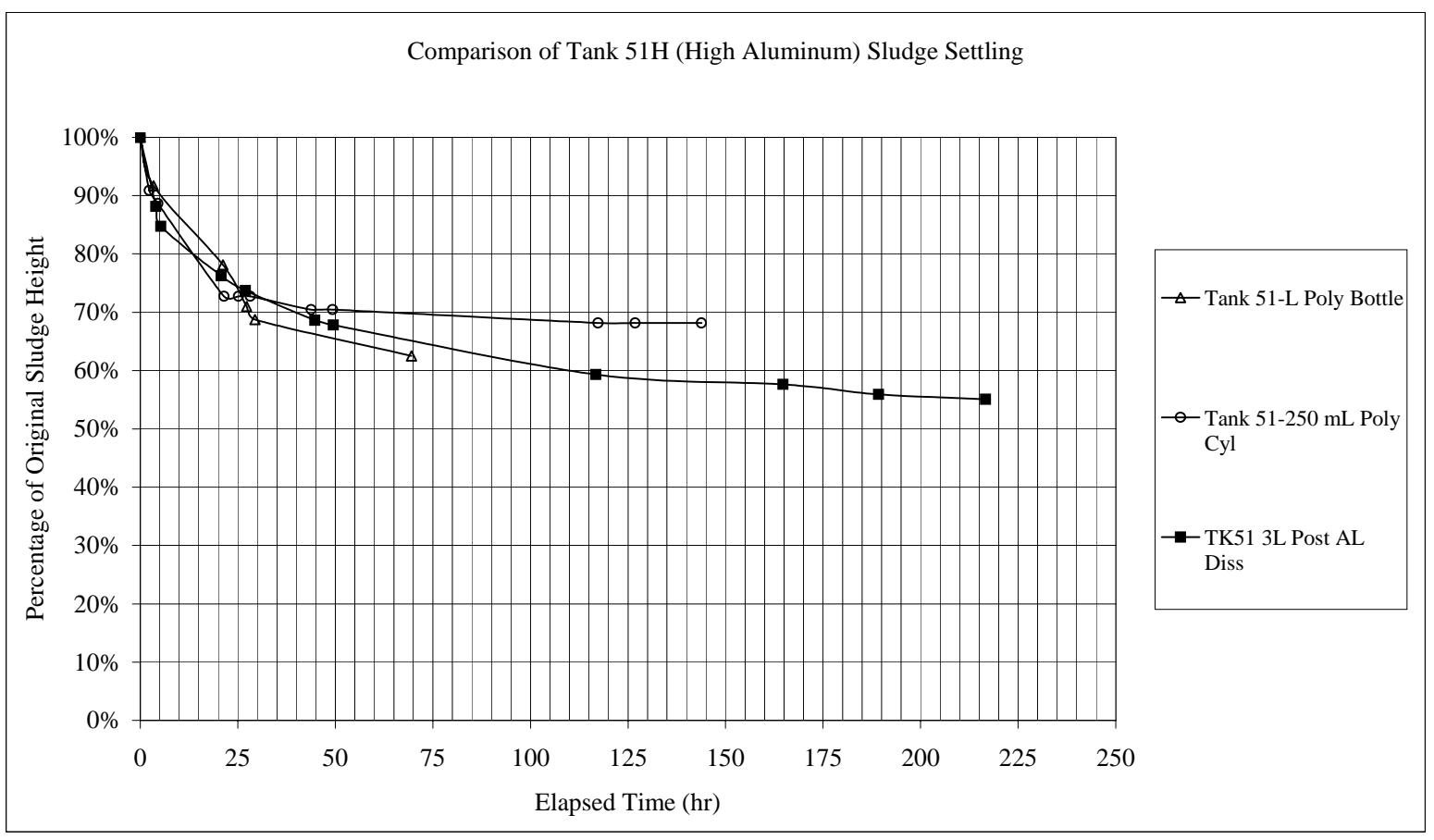

Figure 4.3 Comparison of Sludge Settling for High Aluminum Sludge 


\subsection{POST ALUMINUM DISSOLUTION SLUDGE AND SUPERNATE CHARACTERIZATION}

Table 4-2 summarizes the composition of the key components of the post aluminum dissolution sludge using the data from aqua regia and peroxide fusion dissolution of the total dried solids in Tables 3-9 and 3-10. Due to the large addition of concentrated sodium hydroxide for aluminum dissolution, sodium becomes the most abundant metal in the total solids of the sludge. The aluminum dissolution significantly reduced the amount of aluminum in the sludge solids.

Table 4-2. Summary of the Post Aluminum Dissolution Tank 51H Sludge Composition

\begin{tabular}{|l|c|c|c|}
\hline Analyte & Average & \%RSD & $\begin{array}{c}\text { No. of Values } \\
\text { Averaged }\end{array}$ \\
\hline $\mathrm{Na} \mathrm{mg} / \mathrm{kg}$ & $3.54 \mathrm{E}+05$ & $1.4 \%$ & $4^{\mathrm{a}}$ \\
\hline $\mathrm{Al} \mathrm{mg} / \mathrm{kg}$ & $8.92 \mathrm{E}+04$ & $2.3 \%$ & $4^{\mathrm{b}}$ \\
\hline Fe mg/kg & $5.26 \mathrm{E}+04$ & $1.8 \%$ & 8 \\
\hline $\mathrm{Hg} \mathrm{mg} / \mathrm{kg}$ & $1.45 \mathrm{E}+04$ & $1.3 \%$ & $4^{\mathrm{a}}$ \\
\hline $\mathrm{Mn} \mathrm{mg} / \mathrm{kg}$ & $1.27 \mathrm{E}+04$ & $1.9 \%$ & 8 \\
\hline $\mathrm{U} \mathrm{mg} / \mathrm{kg}$ & $1.14 \mathrm{E}+04$ & $9.9 \%$ & $4^{\mathrm{b}}$ \\
\hline $\mathrm{Ca} \mathrm{mg} / \mathrm{kg}$ & $6.60 \mathrm{E}+03$ & $10 \%$ & 8 \\
\hline $\mathrm{Ni} \mathrm{mg} / \mathrm{kg}$ & $4.33 \mathrm{E}+03$ & $2.6 \%$ & 8 \\
\hline $\mathrm{Si} \mathrm{mg} / \mathrm{kg}$ & $3.09 \mathrm{E}+03$ & $3.4 \%$ & $4^{\mathrm{b}}$ \\
\hline $\mathrm{Mg} \mathrm{mg} / \mathrm{kg}$ & $2.23 \mathrm{E}+03$ & $3.0 \%$ & 8 \\
\hline${ }^{234} \mathrm{U} \mathrm{mg} / \mathrm{kg}$ & $5.16 \mathrm{E}+00$ & $5.9 \%$ & $4^{\mathrm{b}}$ \\
\hline${ }^{235} \mathrm{U} \mathrm{mg} / \mathrm{kg}$ & $9.26 \mathrm{E}+01$ & $5.3 \%$ & $4^{\mathrm{b}}$ \\
\hline${ }^{236} \mathrm{U} \mathrm{mg} / \mathrm{kg}$ & $8.07 \mathrm{E}+00$ & $0.8 \%$ & $4^{\mathrm{b}}$ \\
\hline${ }^{238} \mathrm{U} \mathrm{mg} / \mathrm{kg}$ & $1.12 \mathrm{E}+04$ & $10 \%$ & $4^{\mathrm{b}}$ \\
\hline${ }^{99} \mathrm{Tc} \mathrm{mCi} / \mathrm{kg}$ & $9.77 \mathrm{E}-02$ & $12 \%$ & $4^{\mathrm{b}}$ \\
\hline${ }^{237} \mathrm{~Np} \mathrm{mCi} / \mathrm{kg}$ & $5.74 \mathrm{E}-03$ & $8.3 \%$ & $4^{\mathrm{b}}$ \\
\hline${ }^{238} \mathrm{Pu} \mathrm{mCi} / \mathrm{kg}$ & $2.10 \mathrm{E}+02$ & $6.8 \%$ & $4^{\mathrm{b}}$ \\
\hline${ }^{239 / 240} \mathrm{Pu} \mathrm{mCi} / \mathrm{kg}$ & $5.71 \mathrm{E}+00$ & $7.4 \%$ & $8^{\mathrm{c}}$ \\
\hline${ }^{241} \mathrm{Pu} \mathrm{mCi} / \mathrm{kg}$ & $6.81 \mathrm{E}+01$ & $7.1 \%$ & $4^{\mathrm{b}}$ \\
\hline
\end{tabular}

Divide $\mathrm{mg} / \mathrm{kg}$ values by $1 \mathrm{E}+04$ to convert to $\mathrm{wt} \%$ dried solids basis

a Data taken from the aqua regia dissolution

b Data taken from the sodium peroxide fusion dissolution

c ${ }^{239 / 240} \mathrm{Pu}$ from averaging combined values from ICP-MS and Alpha Counting. 
The large amount of aluminum dissolved from the sludge makes a direct comparison of the as-received and post aluminum dissolution solids compositions difficult. Looking at the ratios of metals to a metal that was not dissolved can provide insight into how the sludge composition changed. The post aluminum dissolution supernate data in Table 3-8 indicate that little or no iron dissolved during the aluminum dissolution. Table 4-3 provides ratios of the concentrations of each of the major metals in the sludge to the iron concentration before and after aluminum dissolution. A ratio that remains constant before and after aluminum dissolution indicates that metal was not removed during the aluminum dissolution.

Only the aluminum to iron and the sodium to iron ratios show significant change due to the aluminum dissolution within the expected uncertainty. The sodium concentration increases due to the large addition of $\mathrm{NaOH}$ at the start of the aluminum dissolution. The aluminum to iron ratio decreases but not by the $\sim 40 \%$ expected based on the amount of aluminum dissolved. Although aluminum dissolution dissolved $\sim 40 \%$ of the aluminum from the sludge, decanting the supernate from the sludge only removes $\sim 20 \%$ of the aluminum from the tank. The other $20 \%$ of dissolved aluminum in the supernate remaining with the sludge must be removed through washing.

The $16 \%$ change in the $\mathrm{Hg} / \mathrm{Fe}$ ratio would seem to be an artifact since no mercury was added to the sample. However, a value for mercury measured in the as-received sludge solids that was biased low for some reason would result in an increased $\mathrm{Hg} / \mathrm{Fe}$ ratio in the post aluminum dissolution sludge. Recall that the analytical results of the supernate phase showed an increased concentration of mercury in the supernate after the aluminum dissolution. This small amount of mercury dissolved from the solids based on the supernate results would be difficult to determine from an analysis of the total dried solids since the small change would fall within the measurement uncertainty. As a result of the obvious problems with the mercury measurement in the total dried solids analysis, the supernate data probably provides a better description of the behavior of mercury during aluminum dissolution.

Table 4-3. Comparison of the Metal to Iron Ratios of the Sludge Solids Before and After Aluminum Dissolution

\begin{tabular}{|c|c|c|c|}
\hline & $\begin{array}{c}\text { As-Received } \\
\text { Sludge }\end{array}$ & $\begin{array}{c}\text { Post Aluminum } \\
\text { Dissolution Sludge }\end{array}$ & \% Change \\
\hline $\mathrm{Al} / \mathrm{Fe}$ & 2.14 & 1.69 & $-21 \%$ \\
\hline $\mathrm{Mn} / \mathrm{Fe}$ & 0.24 & 0.24 & $1.3 \%$ \\
\hline $\mathrm{Hg} / \mathrm{Fe}$ & 0.24 & 0.28 & $16 \%$ \\
\hline $\mathrm{U} / \mathrm{Fe}$ & 0.22 & 0.22 & $-3.9 \%$ \\
\hline $\mathrm{Ca} / \mathrm{Fe}$ & 0.11 & 0.13 & $13 \%$ \\
\hline $\mathrm{Ni} / \mathrm{Fe}$ & 0.09 & 0.08 & $-7.4 \%$ \\
\hline $\mathrm{Si} / \mathrm{Fe}$ & 0.06 & 0.06 & $-1.9 \%$ \\
\hline $\mathrm{Mg} / \mathrm{Fe}$ & 0.04 & 0.04 & $4.3 \%$ \\
\hline $\mathrm{Na} / \mathrm{Fe}$ & 2.00 & 6.72 & $236 \%$ \\
\hline
\end{tabular}


The same type of comparison can be conducted on the radionuclides listed in Table 4-1 and 4-2. Table 4-4 again shows fairly constant ratios between the as-received sludge and the post aluminum dissolution sludge within the expected uncertainty. The ${ }^{99}$ Tc ratio shows an increase in concentration in the sludge after aluminum dissolution. However, the comparison of the supernate data before (Table 3-3) and after aluminum dissolution (Table 3-8), and the samples obtained during aluminum dissolution (Table 3-6) do not support this result. The increase in ${ }^{99}$ Tc can probably be attributed to sampling and analytical uncertainty.

Table 4-4. Comparison of Radionuclide to Iron Ratios of the Sludge Solids Before and After Aluminum Dissolution

\begin{tabular}{|l|c|c|c|}
\hline & $\begin{array}{c}\text { As-Received } \\
\text { Sludge }\end{array}$ & $\begin{array}{c}\text { Post Aluminum } \\
\text { Dissolution Sludge }\end{array}$ & \% Change \\
\hline${ }^{234} \mathrm{U} / \mathrm{Fe}$ & $1.1 \mathrm{E}-04$ & $9.8 \mathrm{E}-05$ & $-9.0 \%$ \\
\hline${ }^{235} \mathrm{U} / \mathrm{Fe}$ & $1.7 \mathrm{E}-03$ & $1.8 \mathrm{E}-03$ & $5.7 \%$ \\
\hline${ }^{236} \mathrm{U} / \mathrm{Fe}$ & $1.6 \mathrm{E}-04$ & $1.5 \mathrm{E}-04$ & $-1.2 \%$ \\
\hline${ }^{238} \mathrm{U} / \mathrm{Fe}$ & $2.1 \mathrm{E}-01$ & $2.1 \mathrm{E}-01$ & $2.1 \%$ \\
\hline${ }^{99} \mathrm{Tc} / \mathrm{Fe}$ & $1.3 \mathrm{E}-06$ & $1.9 \mathrm{E}-06$ & $44 \%$ \\
\hline${ }^{237} \mathrm{~Np} / \mathrm{Fe}$ & $1.0 \mathrm{E}-07$ & $1.1 \mathrm{E}-07$ & $6.2 \%$ \\
\hline${ }^{238} \mathrm{Pu} / \mathrm{Fe}$ & $4.4 \mathrm{E}-03$ & $4.0 \mathrm{E}-03$ & $-8.2 \%$ \\
\hline${ }^{239 / 240} \mathrm{Pu} / \mathrm{Fe}$ & $1.0 \mathrm{E}-04$ & $1.1 \mathrm{E}-04$ & $4.7 \%$ \\
\hline${ }^{241} \mathrm{Pu} / \mathrm{Fe}$ & $1.4 \mathrm{E}-03$ & $1.3 \mathrm{E}-03$ & $-10 \%$ \\
\hline
\end{tabular}

Note: Ratios were calculated using the actual values in Tables 4-1 and 4-2 in the units reported.

The stability of the supernate was observed over several weeks alone and as a mixture with a Tank 11 simulated supernate. For both solutions no precipitation was observed.

\subsection{CONCLUSIONS}

The characterization of the as-received 3-liter sample of Tank $51 \mathrm{H}$ sludge slurry shows a typical high aluminum HM sludge. The XRD analysis of the dried solids indicates Boehmite is the predominant crystalline form of aluminum in the sludge solids. However, amorphous phases of aluminum present in the sludge would not be identified using this analytical technique.

The low temperature $\left(55^{\circ} \mathrm{C}\right)$ aluminum dissolution process was effective at dissolving aluminum from the sludge. Over a three week test, $\sim 42 \%$ of the aluminum was dissolved out of the sludge solids. The process appears to be selective for aluminum with no other metals dissolving to any appreciable extent. At the termination of the three week test, the aluminum concentration in the supernate had not leveled off indicating more aluminum could be dissolved from the sludge with longer contact times or higher temperatures. The slow 
aluminum dissolution rate in the test may indicate the dissolution of the Boehmite form of aluminum however; insufficient kinetic data exists to confirm this hypothesis.

The aluminum dissolution process appears to have minimal impact on the settling rate of the post aluminum dissolution sludge. However, limited settling data were generated during the test to quantify the effects. The sludge settling was complete after approximately twelve days.

The supernate decanted from the settled sludge after aluminum dissolution appears stable and did not precipitate aluminum over the course of several months. A mixture of the decanted supernate with Tank 11 simulated supernate was also stable with respect to precipitation. 


\subsection{REFERENCES}

1. C. J. Bannochie, M. S. Hay, J. M. Pareizs, D. K. Peeler, “Technology Demonstration of Sludge Mass Reduction via Aluminum Dissolution: Task Technical and Quality Assurance Plan” WSRC-RP-2007-00512, Rev. 0, August 2007.

2. J. A. Pike, J. M. Gillam, "Flowsheet for Aluminum Removal from Sludge Batch 5”, LWO-PIT-2007-00042, Rev. 2, September 26, 2007.

Note: The current work was based on revision 1 of the flowsheet. A second revision of the flowsheet document was issued after the start of the aluminum dissolution test.

3. C. J. Bannochie, M. S. Hay, “Analytical Study Plan: Demonstration of Dissolution of Tank 51 Sludge” WSRC-RP-2007-00396, Rev. 1, September 12, 2007.

4. C. J. Coleman, R. A. Dewberry, M. F. Bryant, J. J. Gemmill, SRL's Performance in Round Robin \#6 - Analysis of Simulated Defense Waste Glass (U), WSRC-TR-91187, Rev. 0, May 31, 1991.

5. M. S. Hay, J. M. Pareizs, C. J. Bannochie, M. E. Stone, D. R. Click, D. J. McCabe, "Preliminary Data from the 3L Tank 51 H Aluminum Dissolution Test", SRNL-CST2007-00102, Rev. 0, October 17, 2007.

6. S. H. Reboul, M. S. Hay, J. M. Pareizs, C. J. Bannochie, M. E. Stone, D. R. Click, D. J. McCabe, “Analytical Data for Post-Aluminum Dissolution Decant Solution”, SRNL-PSE-2007-00261, Rev. 0, November 27, 2007.

7. M. S. Hay, T. B. Edwards, Statistical Analysis of ESP Verification Test Samples, WSRC-RP-94-1224, November 4, 1994.

8. M. S. Hay, T. L. Fellinger, Characterization and Settling Tests with Tank 51H Slurry Samples HTF-076-81, WSRC-TR-2005-00525, January 9, 2006.

9. R. E. Eibling, B. A. Hamm, "Demonstration of In-Tank Sludge Processing, Part III: Effect of Processing on Minor Waste Components”, DPST-84-795, October 1, 1984.

10. Personal Communication from R. A. Peterson on recent aluminum dissolution tests using pure samples of Boehmite at temperatures near the temperature used in the study in this report. The model developed by PNNL for Boehmite dissolution has been published:

R. A. Peterson, G. J. Lumetta, B. M. Rapko, and A. P. Poloski, "Modeling of Boehmite Leaching from Actual Hanford High-Level Waste Samples”, Separation Science and Technology, 42: 1719-1730, 2007. 